\title{
Fungal Indole Alkaloid Biogenesis through Evolution of a Bifunctional Reductase/Diels-
}

Alderase

Qingyun Dan ${ }^{1,2, \#}$, Sean A. Newmister ${ }^{1, \#}$, Kimberly R. Klas ${ }^{3}$, James D. Sunderhaus ${ }^{3}$,Amy E. Fraley ${ }^{1,4}$, Jennifer M. Finefield ${ }^{3}$, Amber D. Somoza ${ }^{3}$, Timothy J. McAfoos ${ }^{3}$, Ying Ye ${ }^{1}$, Vikram V. Shende ${ }^{1,5}$, Fengan Yu ${ }^{1}$, Jacob N. Sanders ${ }^{6}$, W. Clay Brown ${ }^{1}$, Le Zhao ${ }^{3}$, Robert S. Paton ${ }^{3}$, K.N. Houk ${ }^{6}$, Janet L. Smith ${ }^{1,2}$, David H. Sherman ${ }^{1,4, *}$ and Robert M. Williams ${ }^{3,7, *}$

${ }^{1}$ Life Sciences Institute, University of Michigan, Ann Arbor, Michigan 48109, USA;

${ }^{2}$ Department of Biological Chemistry, University of Michigan, Ann Arbor, Michigan 48109, USA;

${ }^{3}$ Department of Chemistry, Colorado State University, Fort Collins, Colorado 80523, USA;

${ }^{4}$ Departments of Medicinal Chemistry, Microbiology \& Immunology, and Chemistry, University of Michigan, Ann Arbor, Michigan 48109, USA;

${ }^{5}$ Program in Chemical Biology, University of Michigan, Ann Arbor, Michigan 48109, USA;

${ }^{6}$ Department of Chemistry and Biochemistry, University of California, Los Angeles, California, 90095, USA;

${ }^{7}$ University of Colorado Cancer Center, Aurora, Colorado 80045, USA.

\#These authors contributed equally to this work.

*Robert M. Williams: robert.williams@colostate.edu; David H. Sherman: davidhs@umich.edu. 


\begin{abstract}
Prenylated indole alkaloids isolated from various fungi possess great structural diversity and pharmaceutical utility. Among them are the calmodulin inhibitory malbrancheamides and paraherquamides, used as anthelmintics in animal health. Herein, we report complete elucidation of the malbrancheamide biosynthetic pathway accomplished through complementary approaches. These include a biomimetic total synthesis to access the natural alkaloid and biosynthetic intermediates in racemic form, and in vitro enzymatic reconstitution that provides access to the natural antipode (+)-malbrancheamide. Reductive cleavage of a L-Pro-L-Trp dipeptide from the MalG nonribosomal peptide synthetase (NRPS) followed by reverse prenylation and a cascade of post-NRPS reactions culminates in an intramolecular [4+2] heteroDiels-Alder (IMDA) cyclization to furnish the bicyclo[2.2.2]diazaoctane scaffold. Enzymatic assembly of optically pure (+)-premalbrancheamide involves an unexpected zwitterionic intermediate where MalC catalyzes enantioselective cycloaddition as a bifunctional NADPHdependent reductase/Diels-Alderase. Crystal structures of substrate and product complexes together with site-directed mutagenesis and molecular dynamics simulations demonstrated how MalC and PhqE, its homolog from the paraherquamide pathway, catalyze diastereo- and enantioselective cyclization in the construction of this fascinating class of secondary metabolites.
\end{abstract}


Prenylated indole alkaloids comprised of the bicyclo[2.2.2]diazaoctane core have attracted considerable interest due to their wide spectrum of biological activities and offer compelling targets for chemical synthesis and biosynthetic studies ${ }^{1-3}$. Among them, 2-deoxy-paraherquamide A (derquantel) is a commercially available therapeutic agent for treating parasitic nematodes in sheep ${ }^{4-6}$. It is now clear that in various genera of fungi, two distinct families containing a bicyclo[2.2.2]diazaoctane system have evolved: (1) the dioxopiperazines such as the anti-cancer stephacidins, insecticidal brevianamides and cytotoxic notoamides, and (2) the monooxopiperazines, including the anthelmintic paraherquamides, asperparalines and calmodulin-inhibitory malbrancheamides (Fig. 1a, 1-5). In addition, a series of alkaloids that are putatively derived by the decarbonylative deconstruction of monooxopiperazine progenitors containing the [2.2.2] ring system have been discovered, with the citrinadins representing this structural group $^{7,8}$ (Fig. S1).

The bicyclo[2.2.2]diazaoctane core of these metabolites was first proposed in 1970 to arise from an intramolecular [4+2] Diels-Alder (IMDA) reaction ${ }^{9}$. A long-held hypothesis assumed that both the dioxopiperazine and monooxopiperazine families shared a common biogenesis, with the tryptophan carbonyl of the latter family involved in a net four-electron reduction subsequent to a putative Diels-Alder construction ${ }^{3,10}$. Based on initial genetic studies ${ }^{11}$, and experimental corroboration described in this report, we have discovered that nature employs divergent mechanisms to generate the bicyclo[2.2.2] diazaoctane nucleus in these two distinct families of alkaloids $^{11-15}$ (Fig. S1). Analysis of the malbrancheamide and paraherquamide biosynthetic gene clusters suggested that the bicyclo[2.2.2] diazaoctane ring system is directly produced in the monooxopiperazine oxidation state ${ }^{11}$ (Fig. 1b). We reasoned that it proceeds by the cascade depicted in Figure 1b, 6-12, following reductive cleavage of the tryptophan thiol ester by the nonribosomal peptide synthetase (NRPS) reductase domain ${ }^{11,15}$. The reduced Pro-Trp dipeptide intermediate is reverse-prenylated, and we hypothesized that an intramolecular [4+2] Diels-Alder reaction follows, producing the bicyclo[2.2.2] diazaoctane ring system. However, annotation of the Mal and Phq gene clusters ${ }^{11}$ failed to reveal a candidate enzyme for the IMDA reaction. The putative cycloaddition is stereospecific based on the syn- or anti- configuration of C12a (labeling in premalbrancheamide (1), Fig. 1a) and the relative position of the diene and the dienophile. Antipodal bicyclo[2.2.2]diazaoctanes have been isolated from different fungal strains producing the dioxopiperazine indole alkaloid family, while only $(+)$-malbrancheamide $((+)-2)$ has been isolated from Malbranchea aurantiaca ${ }^{16}$, indicating strict diastereo- and enantioselectivity of the biosynthetic IMDA. Thus, the identification and characterization of this presumed catalytic step is fundamental for understanding the formation of these structurally diverse molecules.

Reports of Diels-Alderases remain rare, with few examples over the past decade ${ }^{17-23}$. Among them, four crystal structures have been reported including, 1) the $S$-adenosyl-L-methionine (SAM)-dependent methyltransferase $\mathrm{SpnF}^{17,24}$, 2) the $\beta$-barrel protein PyrI4 ${ }^{25}$ and its homolog $\mathrm{AbyU}^{26}$, and 3) the flavin-dependent enzyme PyrE3 ${ }^{27}$. A common theme in these Diels-Alderases is their apparent evolution from divergent ancestors, with evident active site reconfiguration. Accordingly, in all reported cases these enzymes have lost ancestral function and the sole 
remaining activity facilitates a spontaneous [4+2] pericyclic reaction with regio- and stereoselectivity. Cofactors, if present, do not serve their canonical catalytic role for the DielsAlder cycloaddition, but rather play a structural role in maintaining the active site in a catalytically productive conformation. Similarly, distinct catalytic residues that abolished the enzymatic function were not identified in any previously characterized Diels-Alderase, suggesting that catalysis is achieved primarily through substrate positioning in the protein scaffold. The malbrancheamide and paraherquamide gene clusters lack homologous genes that encode known Diels-Alderases, which indicated the existence of a novel class of biocatalysts. In this article, we reveal the molecular basis for stereocontrolled construction of the monooxopiperazine bicyclic core in the malbrancheamide and paraherquamide biosynthetic pathways. These genetically homologous systems proceed through a bifunctional reductase and Diels-Alderase that evolved from an ancestral short-chain dehydrogenase (SDR) and is also encoded in several other fungal natural product biosynthetic gene clusters.

a

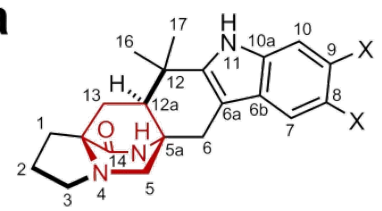

(+)-premalbrancheamide $(+)-1 \mathrm{X}=\mathrm{H}$

$(+)$-malbrancheamide $(+)-2 \mathrm{X}=\mathrm{Cl}$

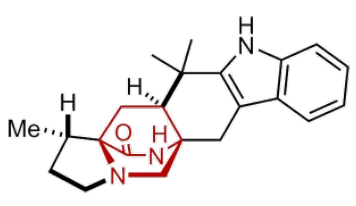

$(+)$-preparaherquamide $(+)-3$

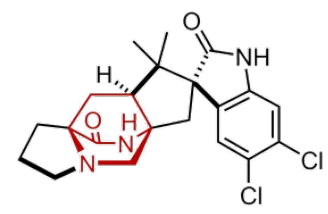

(-)-spiromalbramide (-)-4

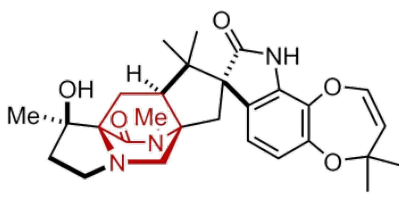

(-)-paraherquamide A (-)-5

b
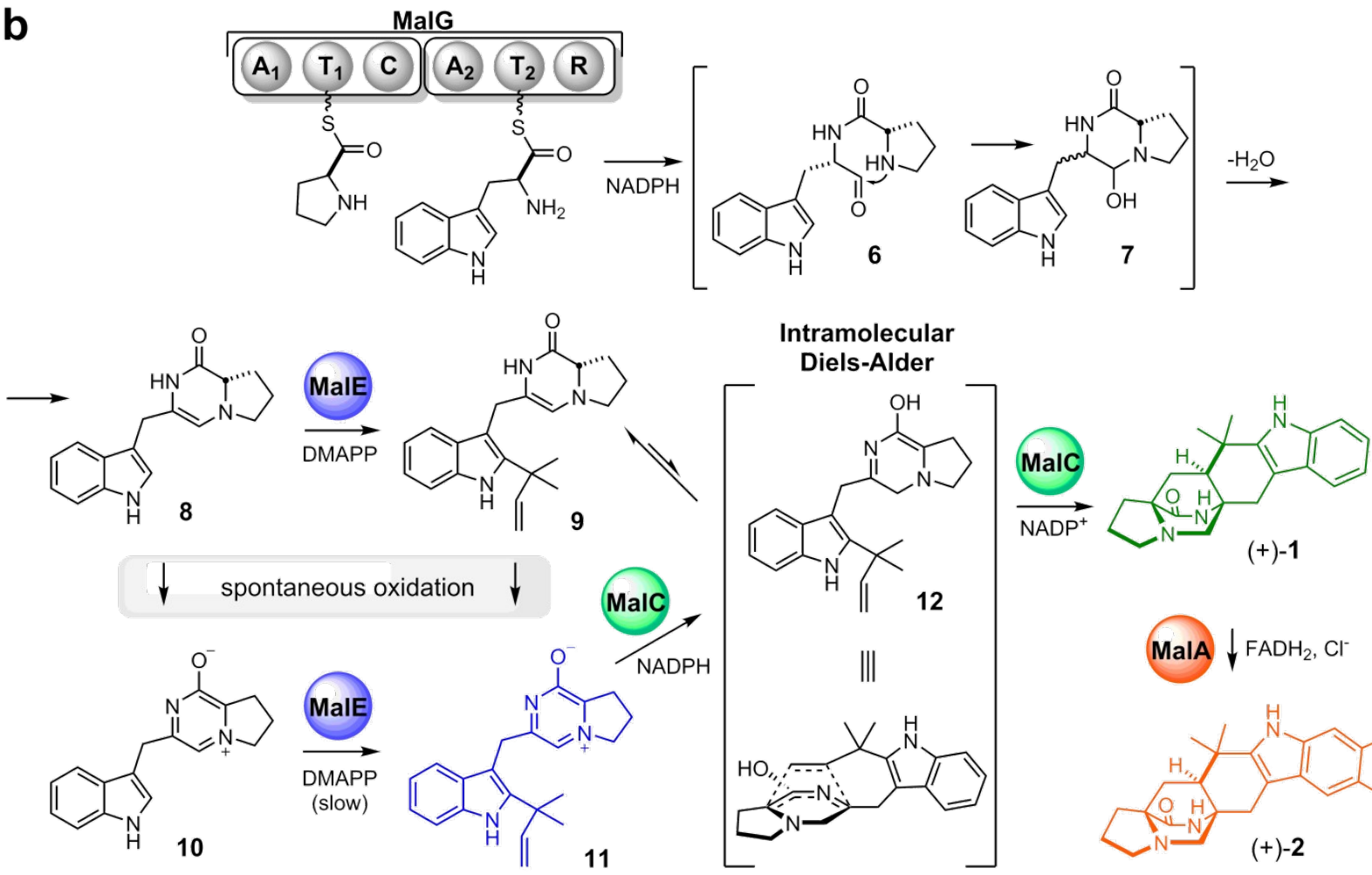

11

Figure 1. Fungal bicyclo[2.2.2]diazaoctane indole alkaloids and biosynthesis. 
a. Representative natural products with the biocyclo[2.2.2]diazaoctane group colored in red. b. Scheme of malbrancheamide biosynthesis. The natural substrates are L-proline and L-tryptophan, and the final product is malbrancheamide (+)-2. The product of each biosynthetic step is colored differently. Proteins are indicated by spheres; MalG domains are adenylation $\left(\mathrm{A}_{1}\right.$ and $\left.\mathrm{A}_{2}\right)$, thiolation $\left(\mathrm{T}_{1}\right.$ and $\left.\mathrm{T}_{2}\right)$, condensation $(\mathrm{C})$ and reductase $(\mathrm{R})$.

\section{Biomimetic Synthesis of Premalbrancheamide, Malbrancheamide and Spiromalbramide}

Early considerations regarding biogenesis of the bicyclo[2.2.2] diazaoctane core envisioned a biosynthetic Diels-Alder reaction. In order to chemically validate the sequence of events in malbrancheamide biosynthesis, we prepared the $\mathrm{C} 2$ reverse prenylated proposed biosynthetic intermediate, dipeptide aldehyde $(\mathbf{1 7})^{11}$, and found that this substance undergoes the cascade of ring closure, dehydration, tautomerization and intramolecular cycloaddition, upon deprotection to give premalbrancheamide (1) (Fig. 2). This strategy was applied to two additional natural products, malbrancheamide (2) and spiromalbramide (4) (Fig. S2), underscoring the utility of the biomimetic paradigm. The key, Fmoc-protected dipeptide aldehyde 17 was prepared through the peptide coupling of N-Fmoc proline (14) with the C2 reverse prenylated tryptophan methyl ester 13 through the agency of HATU in acetonitrile in $85 \%$ yield. Reduction of the methyl ester with sodium borohydride ( $82 \%$ to 15 ) followed by a Doering-LaFlamme oxidation, furnished the $\mathrm{N}$ Fmoc aldehyde 17 in $72 \%$ yield. Removal of the N-Fmoc residue with diethylamine under anaerobic conditions provided the di-enamine 9, which could be isolated and characterized. Treatment of this substance with TFA in THF at temperatures between $0{ }^{\circ} \mathrm{C}$ and $50{ }^{\circ} \mathrm{C}$, resulted in formation of $( \pm)-1$, and the unexpected carbinolamine oxidation congener 14 and 15 as minør products. At $50{ }^{\circ} \mathrm{C}$, premalbrancheamide was produced in an $8: 1$ ratio with $\mathbf{1 4}$ and $\mathbf{1 5}$ (inseparable) in $40 \%$ combined yield. The observed modest yield is possibly due to unfavorable tautomerization of 9 to $\mathbf{1 2}$. Under aerobic conditions, 9 spontaneously and rapidly oxidized to aromatic zwitterion 11 (Fig. 1b), which we initially reasoned to be a non-physiological byproduct. We later determined that 11 could be chemically reduced by $\mathrm{NAD}(\mathrm{P}) \mathrm{H}$ to 12, resulting in the spontaneous formation of racemic premalbrancheamide in $80-90 \%$ combined yield. This discovery suggested two possible biosynthetic routes - aerobic vs. anaerobic - and the possibility that $\mathbf{1 1}$ is an authentic pathway intermediate depending on the intracellular conditions during fungal biosynthesis. Significantly, both routes lead to a single syn-diastereomer upon cyclization as the corresponding anti-isomer was not detected in even trace amounts from the cycloaddition reactions. This finding agrees with density functional theory calculations that the azadiene $\mathbf{1 2}$ has a calculated relative transition state energy difference of about $2.6 \mathrm{kcal} / \mathrm{mol}$ favoring the syncycloadduct $^{28}$. The anti-pathway experiences unfavorable steric interactions between the pyrrolidine ring and the prenyl group (Fig. S3). Our biomimetic synthesis of synmalbrancheamides gave rise to the $(+)$ - and $(-)$ - enantiomers, raising the intriguing question regarding how optically pure $(+)$-premalbrancheamide is formed by Malbranchea aurantiaca. This further indicated the likely presence of an enzyme-directed cyclization in vivo, and 
motivated us to explore the biosynthetic origins of premalbrancheamide by in vitro pathway reconstitution.

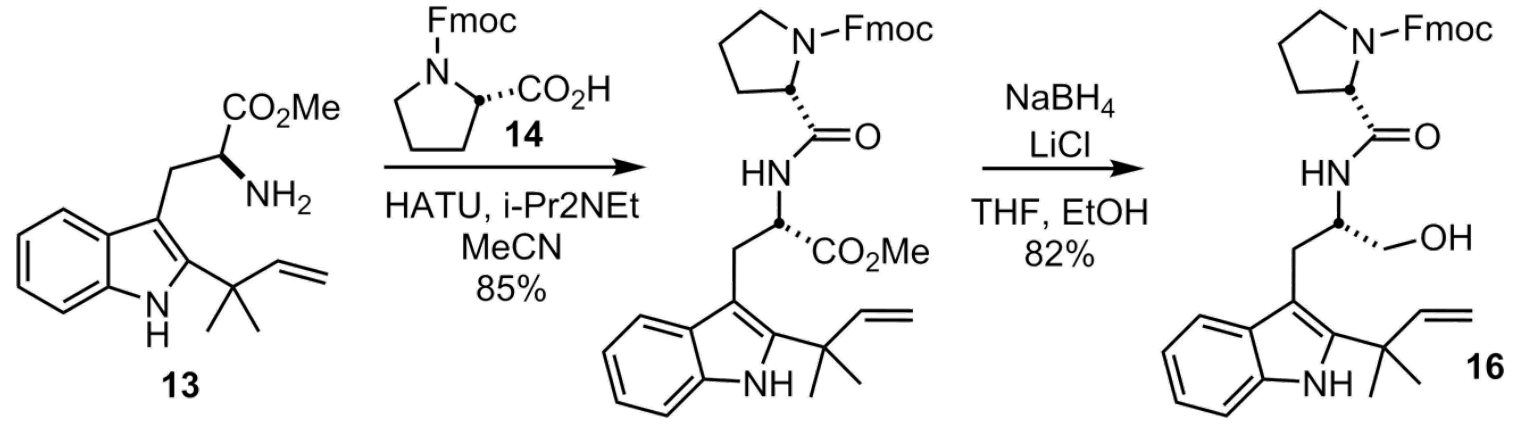

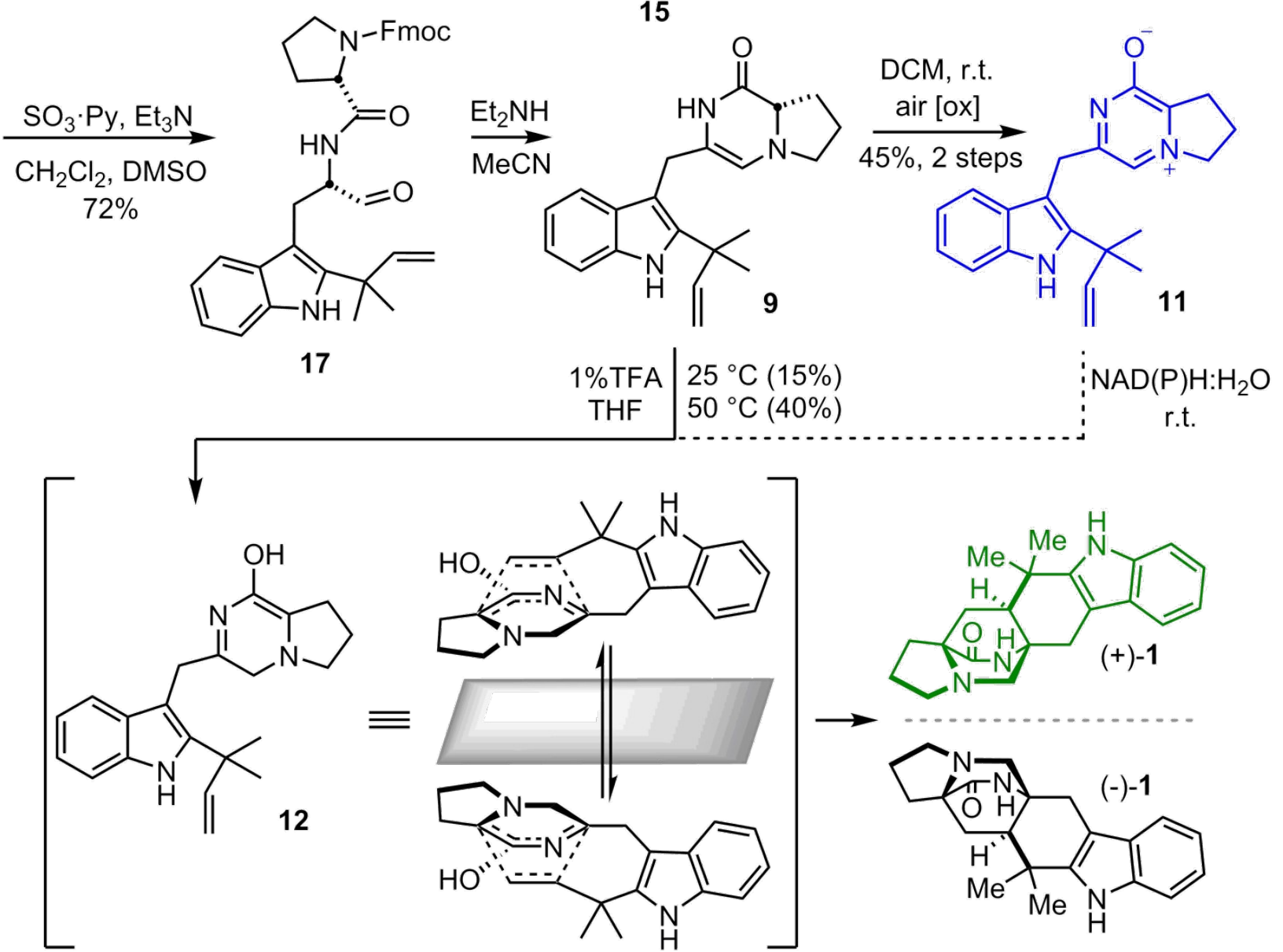

Figure 2. Biomimetic synthesis of premalbrancheamide.

The biomimetic synthesis proceeded through a spontaneous intramolecular [4+2] Diels-Alder reaction from a key azadiene intermediate $\mathbf{1 2}$ to produce a racemic mixture of synpremalbrancheamides (1). Only optically pure (+)-1 has been isolated from Malbranchea aurantiaca. See SI for complete methods. 
We aimed to reconstitute the biosynthesis of malbrancheamide as a representative monooxopiperazine alkaloid in a multi-component in vitro reaction (Fig. S4). We hypothesized the first step of malbrancheamide biosynthesis involves coupling of L-proline and L-tryptophan by MalG, a dimodular NRPS containing six domains $\left(\mathrm{A}_{1}-\mathrm{T}_{1}-\mathrm{C}-\mathrm{A}_{2}-\mathrm{T}_{2}-\mathrm{R}\right.$, Fig. $\left.1 \mathrm{~b}\right)$, to produce LPro-L-Trp aldehyde 6 through reductive off-loading. Since the full-length NRPS protein could not be produced in soluble form, we identified domain boundaries in MalG, developed expression constructs for the excised $A_{1}-T_{1}, C, T_{2}$ and $\mathrm{R}$ domains (Fig. S5), and loaded the putative amino acid substrates onto the MalG $\mathrm{T}_{1}$ and $\mathrm{T}_{2}$ domains (Fig. S6a, b). Phosphopantetheinylated MalG $\mathrm{A}_{1}-\mathrm{T}_{1}$ was loaded with L-proline in the presence of ATP and $\mathrm{Mg}^{2+}$, consistent with our functional annotation. With no access to soluble MalG $\mathrm{A}_{2}$, Ltryptophan was loaded onto $\mathrm{MalG} \mathrm{T}_{2}$ using $\mathrm{Sfp}^{29}$, a nonspecific 4'-phosphopantetheinyl transferase, and L-Trp-coenzyme A (CoA). L-Pro $A_{1}-T_{1}$ and L-Trp $T_{2}$ were incubated with the MalG $\mathrm{C}$ domain and $\mathrm{R}$ domain with the presumed NADPH cofactor. Product formation was determined by LC/MS and comparison with authentic standards. Instead of the proposed dipeptidyl aldehyde-derived product $\mathbf{8}$, we identified aromatic zwitterion $\mathbf{1 0}$ as the main product (Fig. $1 \mathrm{~b}$ and $3 \mathrm{a}$ ). We hypothesized that $\mathbf{1 0}$ was produced from spontaneous oxidation of $\mathbf{8}$. This was confirmed by chemical synthesis of $\mathbf{8}$, which spontaneously and irreversibly converted to $\mathbf{1 0}$. This transformation was suppressed under anaerobic conditions, leading to the conclusion that the malbrancheamide NRPS product rapidly cyclized and dehydrated to $\mathbf{8}$ and subsequently spontaneously oxidized to $\mathbf{1 0}$ under aerobic (i.e. physiological) conditions.

To further test the hypothesis that the MalG terminal R-domain catalyzes an NADPHdependent two-electron reductive release to produce an aldehyde, we synthesized a dipeptidylCoA analog 23, in which the prolyl-N-atom was replaced with an O-atom to prevent nucleophilic addition of the prolyl-N-atom to the CoA thioester, and loaded 23 onto MalG $\mathrm{T}_{2}$ via Sfp (Fig. S6c). Product standards of aldehyde 24 and alcohol 25 were synthesized chemically. Compound 25 was nonreactive in methanol, while 24 epimerized and reacted to produce the hemiacetal 26 (Fig. S7). Assays with 23-loaded $\mathrm{T}_{2}$ and MalG $\mathrm{R}$ yielded product 26, confirming that MalG generates an aldehyde product. NADPH was the preferred cofactor in this reaction (Fig. S7d, e). MalG $\mathrm{R}$ is an SDR reductase with catalytic Tyr and Lys amino acids, as demonstrated in the 2.6$\AA$ crystal structure of an NADPH complex of PhqB R, the MalG R homolog of paraherquamide biosynthesis (Figs. S8-10). The essential role of Tyr was confirmed with MalG R/Y2132F, which was incapable of reductive release (Fig. S10d).

We propose that the NRPS product 8 would undergo a reverse-prenylation as the next biosynthetic step, thereby installing the dienophile for the IMDA reaction. Two genes, malE and malB (from the mal gene cluster) encode putative prenyltransferases. We incubated MalB or MalE with substrate-loaded MalG domains, NADPH and dimethylallyl pyrophosphate (DMAPP), the prenyl donor. MalE readily catalyzed a C2 reverse prenyl transfer reaction to produce zwitterion 11 (Fig. 3b), whereas MalB displayed modest prenylation activity, suggesting that malB may be a redundant gene in the pathway (Fig. 3c and S14). Because we could not distinguish in this assay whether $\mathbf{8}$ or $\mathbf{1 0}$ was the MalE substrate, synthetic $\mathbf{8}$ was produced under 
anaerobic conditions by UV irradiation of an $O$-nitrobenzyl (ONB) photo-protected dipeptide aldehyde $\mathbf{3 0}$ and subjected to the prenyltransferase assay (Fig. S11a). This substrate was rapidly prenylated by MalE in contrast to synthetic 10, which showed only low levels of conversion with the enzyme, indicating that $\mathbf{8}$ is the native substrate for MalE (Fig. S12). This raised the question regarding how MalE accesses substrate $\mathbf{8}$ prior to its rapid oxidation to 10. Thus, we considered the possibility that $\mathrm{C} 2$ reverse prenylation occurs with the substrate tethered to the NRPS $\mathrm{T}_{2}$ domain. To address this question, we tested whether MalE or MalB could prenylate L-Trp, LTrp-loaded MalG $\mathrm{T}_{2}$, or 23-loaded MalG $\mathrm{T}_{2}$ (Fig. S13). In all cases, no prenylated product was detected, confirming that the prenyl transfer reaction occurred on free substrate following the NRPS-catalyzed reaction. 
a

MalG

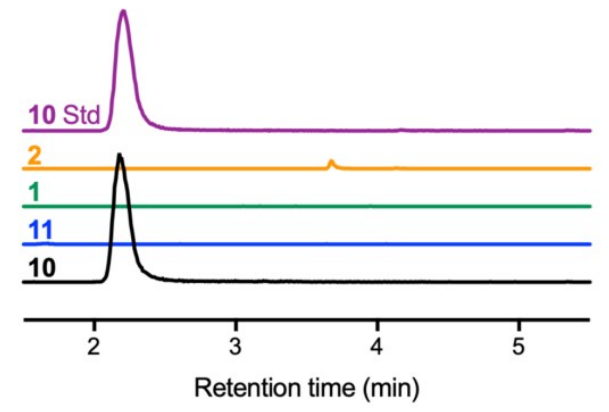

C

MalG+MalB

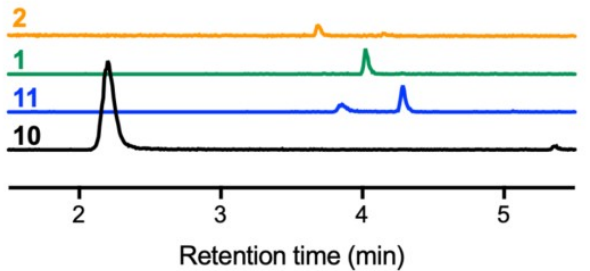

e

MalG+MalE+MalC+MalA

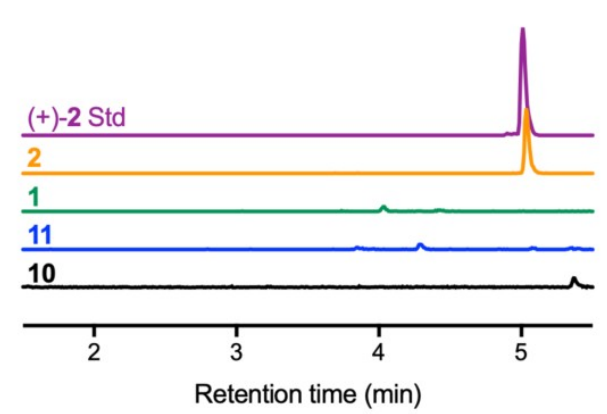

g

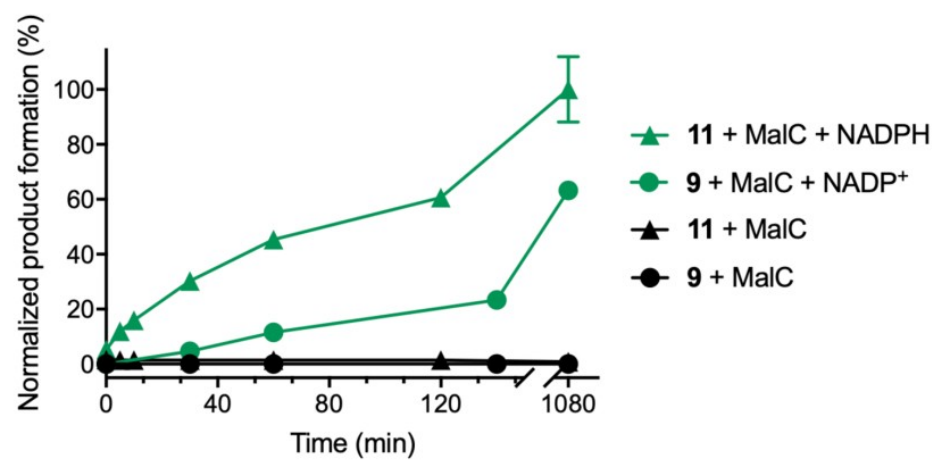

MaIG+MalE

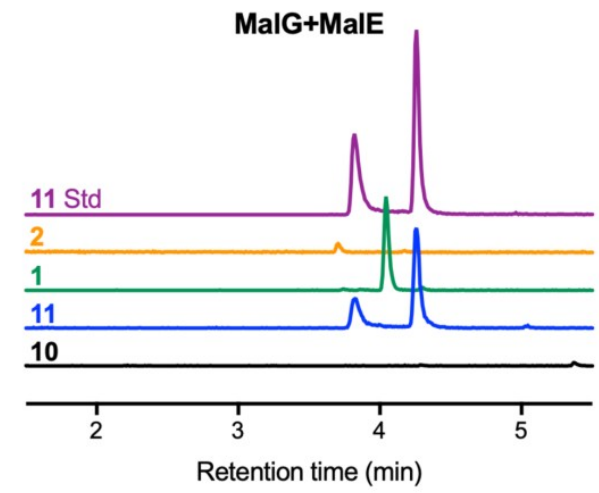

d

MalG+MalE+MalC

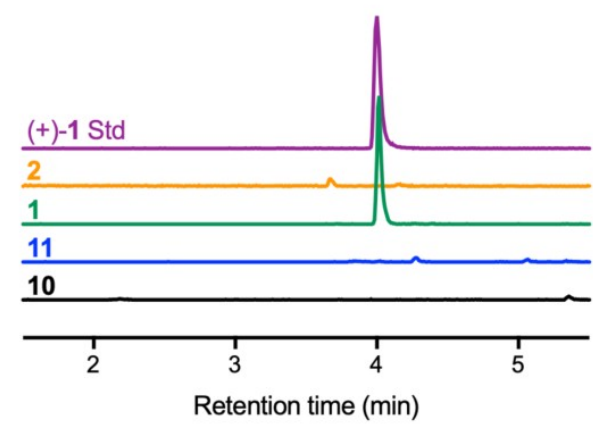

f Chiral Separation of (+)-1 and (-)-1

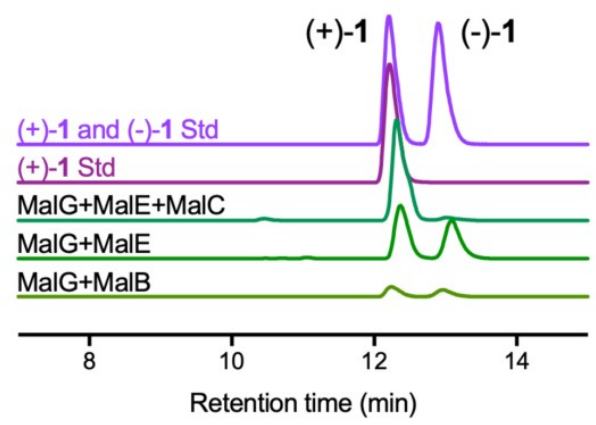

h

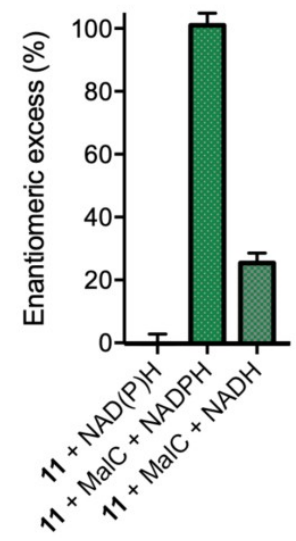




\section{Figure 3. In vitro enzymatic reconstitution of malbrancheamide biosynthesis.}

Reactions were monitored by LC/MS. Extracted ion counts (EIC) for key molecules in reaction mixtures are compared to authentic synthetic standards. a. MalG NRPS produced zwitterion 10 by spontaneous oxidation of 8 . $b-c$. Addition of MalE or MalB prenyltransferase formed three products: a prenylated zwitterion 11, and $( \pm)$-1. d. MalC Diels-Alderase addition disabled formation of 11 and (-)-1 (see panel f). e. Malbrancheamide 2, the final pathway product, was produced by MalA halogenation of $(+)-\mathbf{1}$. $\mathrm{f}$. Chiral separation of $( \pm)-\mathbf{1}$ indicates that MalC is an intramolecular [4+2] Diels-Alderase, while MalE or MalB does not provide enantioselectivity for the spontaneous IMDA reaction. g. MalC-catalyzed reactions under aerobic $(\mathbf{1 1}+\mathrm{MalC})$ or anaerobic $(\mathbf{9}+\mathrm{MalC})$ conditions. The aerobic route with 11 as the pathway intermediate was more efficient than the anaerobic route from 9. h. Effect of cofactor on the enantiomeric excess of the MalC-catalyzed Diels-Alder reaction. MalC provided limited enantioselectivity when NADH was used as cofactor. EIC traces are colored by compound as in Figure $1 \mathrm{~b}$, authentic standards are in purple or pink. For panel $g$ and $h$, all data represent the average of triplicate measurements (error bars, $\mathrm{SD} ; n=3$ ).

We noticed low levels of premalbrancheamide in the reconstitution assays with MalG and MalE or MalB. Chiral LC/MS analysis revealed a 1:1 racemic mixture of $( \pm$ )-1 (Fig. 3f), in agreement with the biomimetic synthesis described above (Fig. 2). Further investigation using synthetic 11 revealed that racemic premalbrancheamide arose through non-enzymatic reduction of $\mathbf{1 1}$ by NADPH to azadiene 12, which undergoes spontaneous cycloaddition in the reaction buffer, thereby explaining the background accumulation of the Diels-Alder products $( \pm)-\mathbf{1}$ from in vitro assays. From these studies we ascertained that MalG and MalE are the minimal components required for premalbrancheamide biosynthesis, albeit lacking stereocontrol in the IMDA reaction.

Premalbrancheamide isolated from Malbranchea aurantiaca is optically pure (+)-1, which strongly implicates enzymatic control in the IMDA reaction. Known Diels-Alderases have diverse origins, but the annotated $\mathrm{mal}$ and $\mathrm{phq}$ gene clusters did not contain an evident candidate biosynthetic enzyme. Nonetheless, we tested whether $\mathrm{MalC}$, annotated as a short-chain dehydrogenase/reductase (SDR), could function as the presumed Diels-Alderase. When MalC was incubated with substrate-loaded MalG and MalE (NADPH and DMAPP included), neither aromatic zwitterion intermediate, $\mathbf{1 0}$ or 11, was detected; instead the sole product was $(+)-\mathbf{1}$, confirming that MalC functions as an intramolecular [4+2] Diels-Alderase (Fig. 3d). To our surprise, when MalC was added to the reaction mixture after significant amounts of $\mathbf{1 1}$ had accumulated, the oxidized intermediate was converted to (+)-1, indicating that MalC possessed the ability to reduce the zwitterion $\mathbf{1 1}$ to the reactive azadiene $\mathbf{1 2}$ prior to conducting the diastereo- and enantio-controlled cycloaddition reaction. This unexpected reactivity of MalC was confirmed using synthetic 11 and NADPH (Fig. S15). To our knowledge, this is a unique example where reduction regenerates the biosynthetic substrate from an oxidized (aromatic) intermediate to provide a productive mode for cycloaddition. The fact that $\mathbf{1 1}$ is a MalC substrate 
indicates that it is an authentic pathway intermediate, and motivated us to address whether an aerobic or anaerobic biosynthetic route is operative in vivo. This question was interrogated in two ways; first by performing MalC assays under anaerobic conditions with synthetic $\mathbf{9}$, which was generated by photo-deprotection of ONB prenyl dipeptidyl aldehyde 33 (Fig. S11b). Conversion to (+)-1 was observed only in the presence of $\mathrm{MalC}$ and $\mathrm{NADP}^{+}$(Fig. 3g). However, the efficiency of this reaction was attenuated compared to the MalC-catalyzed conversion of $\mathbf{1 1}$ to $(+)-\mathbf{1}$ under aerobic conditions, indicating that the dienamine tautomer $\mathbf{9}$ is not optimally recognized by MalC. It is unknown whether MalC can play a role in tautomerization of $\mathbf{9}$; notably, background conversion of $\mathbf{9}$ to racemic premalbrancheamide was not detected under these conditions. Second, gene disruption of the malC homolog phqE was conducted in the paraherquamide-producing strain Penicillium simplicissimum using a CRISPR-Cas9 system $^{30}$. Extracts from the $p h q E$ mutant strain grown on CYA medium showed the presence of the expected (methyl-Pro-Trp prenyl) zwitterion intermediate 39 (Fig. S16) by LC/MS analysis and co-injection with a synthetic standard, confirming the accumulation of this oxidized metabolite in vivo (Fig. S17). Taken together, these data indicate that $\mathbf{1 1}$ is the native substrate for MalC en route to $(+)$-premalbrancheamide.

For the MalC-catalyzed reduction of 11, either NADH or NADPH could be used as the cofactor. However, NADPH is required for strict stereocontrol of the IMDA reaction, as MalC produced a 63:37 mixture of (+)-1 and (-)-1 when using NADH as cofactor (Fig. 3h). This is consistent with the anaerobic experiment in which $\mathrm{NADP}^{+}$was required to generate $(+)-\mathbf{1}$, and further indicates that the cofactor NADPH plays an important role in the IMDA stereocontrol. The MichaelisMenten kinetic constants for NADPH and NADH in reactions with MalC and $\mathbf{1 1}$ revealed a 10fold greater catalytic efficiency $\left(k_{\text {cat }} / \mathrm{K}_{\mathrm{M}}\right)$ with NADPH than with NADH (Fig. S15d, e). A 6-fold greater $\mathrm{K}_{\mathrm{M}}$ with $\mathrm{NADH}$ also suggests that proper cofactor binding is a required component to achieve stereocontrol. Enzymatic rate enhancement of $(+)-\mathbf{1}$ formation is evident under both assay conditions: aerobically through substrate $\mathbf{1 1}$ (post-reduction), or anaerobically through substrate 9 (post-tautomerization) (Fig. 3g). The dramatic shift in enantiomeric excess for the enzymatic reaction (from $0 \%$ to $96 \%$ ) is indicative of enzymatic catalysis for the IMDA reaction.

To complete the biosynthetic pathway, a flavin-dependent halogenase MalA was shown to catalyze two iterative halogenation reactions to add chlorine atoms on $\mathrm{C} 8$ and $\mathrm{C} 9$ of premalbrancheamide $(+)-\mathbf{1}$ to produce malbrancheamide $(+)-2^{31}$. We incubated MalA with its pathway partners (L-Pro and L-Trp MalG, MalE and MalC, NADPH, DMAPP, NaCl and $\mathrm{FADH}_{2}$ ) and identified (+)-2 as the final product (Fig. 3e). We also found that MalA is stereospecific: when incubated with racemic mixture of 1, MalA chlorinated only the natural $(+)$ enantiomer (Fig. S18).

\section{Probing the catalytic mechanism of the bifunctional Diels-Alderase}

To gain further insight into the function of the SDR-derived Diels-Alderase, the crystal structure of ligand-free MalC was solved at 1.6- $\AA$ resolution, revealing a classical SDR fold with a 
nucleotide-binding subdomain that contains an invariant "TGX $\mathrm{GXG}_{3}$ " motif (P-loop), and a Cterminal substrate binding region that is less conserved and largely hydrophobic ${ }^{32,33}$. The closest structural homologs are a group of SDRs including RasADH (2.3 $\AA$ C $\alpha$ R.M.S.D., $27 \%$ overall sequence identity $)^{34}$, which uses $\mathrm{NAD}(\mathrm{P})$ to catalyze reversible oxidation of secondary alcohols to aldehydes. Unexpectedly, MalC lacks the characteristic Tyr and Lys catalytic amino acids, and also the essential Asn and Ser residues of typical SDRs ${ }^{32,33}$, suggesting that the active site is reconfigured to fit its unique catalytic roles.

Neither cofactor nor substrate was captured in complex with MalC due to crystal lattice constraints. Thus, we turned to the homologous paraherquamide biosynthetic pathway. PhqE is a MalC homolog (54\% identity) and catalyzed the same reaction using the zwitterionic prenylated Trp-Pro substrate 11 in vitro (Fig. S19). The 2.4- $\AA$ crystal structure of PhqE in complex with cofactor $\left(\mathrm{NADP}^{+} / \mathrm{NAD}^{+}\right)$and premalbrancheamide (Fig. S20) is highly similar to the MalC structure (1.0 ^ C $\alpha$ R.M.S.D.; Fig. 4b).

Consistent with our kinetic data, PhqE crystals grown with $\mathrm{NADP}^{+}$showed strong electron density for the cofactor (Fig. S21a) and binding in a manner conserved with bacterial SDR homologs (Fig. S21c), while NAD ${ }^{+}$showed weak electron density (Fig. S21b). Lys50 accounts for preferential binding of $\mathrm{NADP}^{+}$through a salt bridge with the cofactor 2'-phosphate. Premalbrancheamide binds in a groove on the surface and is surrounded by hydrophobic residues. The bicyclo[2.2.2] diazaoctane ring system is buried against the nicotinamide and several amino acids including Arg131 (Fig. 4c). The indole lies in a pocket formed by Ala, Leu and Val side chains. The gem-dimethyl contacts Asp166 and Trp169, which are part of a conserved "PDPGW" motif (Fig. S24-25). Given the dual functions (reductase and DielsAlderase) of MalC/PhqE, the product complex reveals PhqE to be well-adapted in its capacity as a stereo- and enantioselective biocatalyst due to the shape complementarity between the active site pocket and the product (Fig. 4f). Additionally, the short distance ( $\sim .4 \AA$ ) between nicotinamide $\mathrm{C} 4$ and the deoxy $\mathrm{C} 5$ of premalbrancheamide suggests that reduction to the reactive azadiene also occurs in the same location of the active site and indicates that reduction and cycloaddition are highly coordinated. 

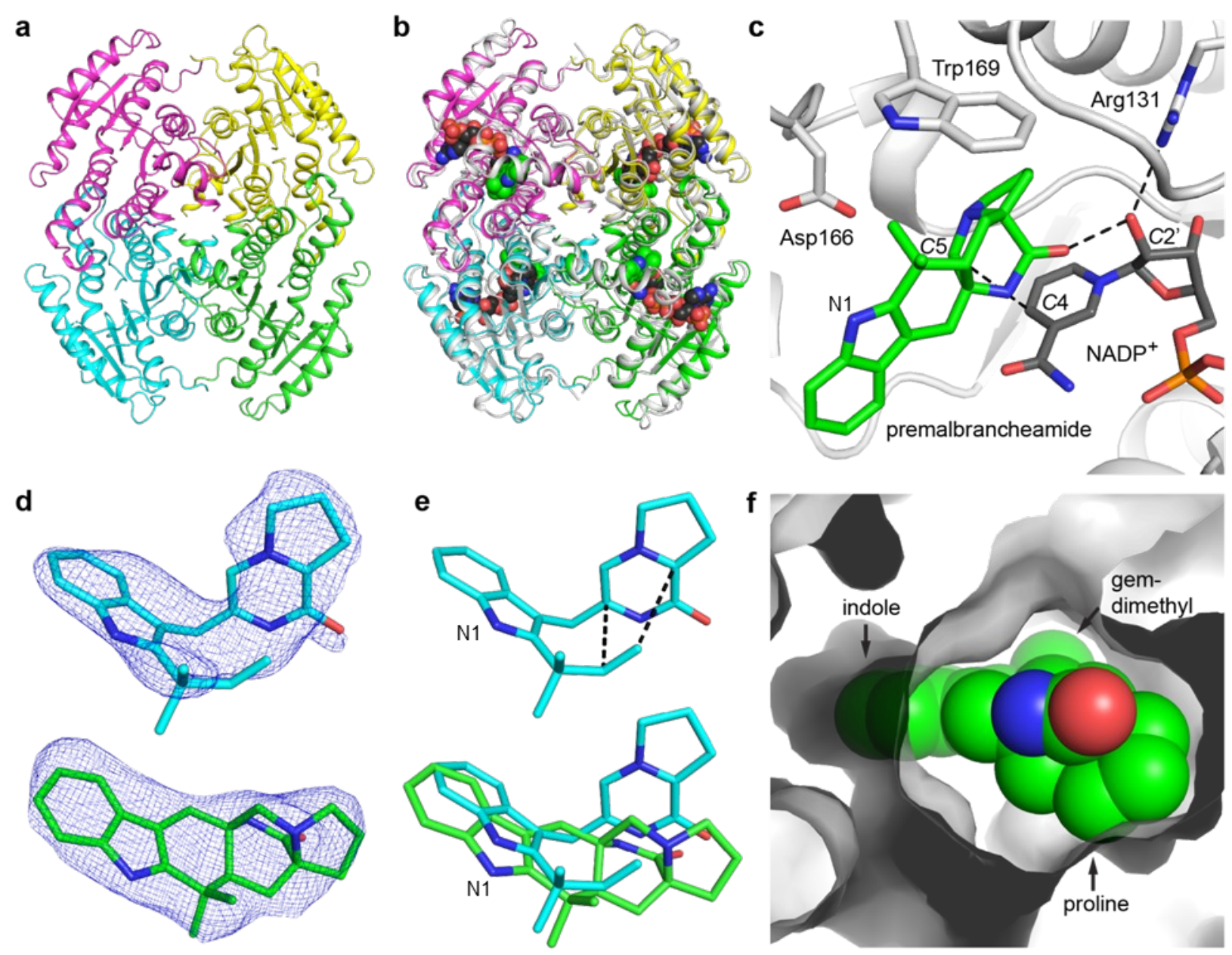

Figure 4. Structures of MalC and PhqE.

a. MalC tetramer colored by subunit. b. Superposition of MalC and PhqE product complex (gray); $\mathrm{NADP}^{+}$(black C) and premalbrancheamide (green C) are shown as spheres. c. PhqE active site showing close arrangement of the product and the $\mathrm{NADP}^{+}$cofactor. d. Omit electron density $\left(\mathrm{F}_{\mathrm{o}}-\mathrm{F}_{\mathrm{c}}\right.$; contoured at $\left.2.2 \sigma\right)$ for the substrate $\mathbf{1 1}$ (cyan) and the premalbrancheamide $(+)-\mathbf{1}$ product (green). e. Pre-organization for cycloaddition. Substrate 11 (upper) binds with the prenyl group poised for the IMDA (dashed lines), as seen in the overlay of $\mathbf{1 1}$ and premalbrancheamide (+)-1 (lower). f. Surface representation of the product complex showing high shape complementarity between premalbrancheamide and PhqE.

We sought a non-reactive substrate complex with $\mathrm{NADP}^{+}$and $\mathbf{1 1}$, which resulted in ambiguous electron density for 11 with the wild-type PhqE. However, PhqE/D166N (described below) showed strong density for 11, with indication of a flexible orientation (Fig. 4d). The indole of 11 binds in the same pocket as premalbrancheamide while the pyrazinone is pushed towards the nicotinamide (Fig. 4e). Deoxy C5 of 11 lies $3.6 \AA$ from the nicotinamide C4 consistent with hydride delivery to this position. In addition to hydride transfer, protonation of the pyrazinone alkoxide is required to form the reactive azadiene 12. Curiously the corresponding oxygen atom 
is part of a hydrogen-bonding network involving the $\mathrm{NADP}^{+} 2^{\prime}$-hydroxyl and Arg131, suggesting that the cofactor may play a role in proton-transfer during reduction. Superposition of the substrate and product complexes revealed a high degree of pre-organization of $\mathbf{1 1}$ towards the Diels-Alder reaction and also affirmed that the reduction and IMDA reactions are spatially confined.

We used molecular dynamics (MD) simulations to explore the concepts of coordinated hydride delivery, proton transfer and pre-organization in the active site. First, we monitored the distance between the putative hydride acceptor (C5 of 11) and nicotinamide $\mathrm{C} 4$ over a $1.2 \mu$ s simulation. The average distance between these carbon atoms was $4.2 \AA$, consistent with the crystal structures (Fig. S22). We next explored alkoxide protonation. In the crystal structure the NADP ${ }^{+}$ ribose 2'-hydroxyl is hydrogen-bonded to the alkoxide oxygen and Arg131. Within the first few ns of the simulation Arg131 displaces the ribose hydroxyl and interacts with the alkoxide for the remainder of the simulation, lending significance to the role of Arg131 in protonation of $\mathbf{1 1}$. To assess facial selectivity in the cycloaddition reaction that forms the $(+)$ and $(-)-$ premalbrancheamide enantiomers, we monitored the dihedral angle along N15-C5a-C12a-C13 (Fig. S23). Comparison of this dihedral angle in the constrained premalbrancheamide and unconstrained 11 revealed that the untethered diene explores only a single face of the pyrazinone ring corresponding to the natural $(+)$ enantiomer (Fig. S23). Interestingly, this pre-organization was lost when $\mathrm{NADP}^{+}$was omitted from the simulation, consistent with our observation that the cofactor is required for enantio-controlled cycloaddition. Together these results further support the conclusion that reduction and cycloaddition are coordinated and take place in the same active site pocket where the enzyme-cofactor complex provides stereocontrol by positioning the diene for [4+2] cycloaddition as the reactive azadiene is generated by reduction of $\mathbf{1 1}$.

Based on this information, we probed the reaction mechanism by site-directed mutagenesis. MalC was chosen for this analysis to directly compare results with the reconstitution assay. All of the targeted amino acid are conserved in MalC and PhqE. With the in vitro reconstitution assay, MalC variants were assayed in the presence of the MalG NRPS and MalE prenyltransferase (Fig. 5a, b). Reductase activity was assessed by the levels of oxidized intermediate 11: higher levels indicate less reductase activity. The effect on the IMDA reaction was determined by measuring levels of unnatural (-)-1 as a percent of all premalbrancheamide: $\sim 50 \%$ (-)-1 formation indicates loss of enzymatic IMDA function. We identified five MalC substitutions that abolished reductase activity (D108A, R130A, D165A, D165N, W168L), and found that loss of reductase activity is highly correlated with loss of stereocontrol in the IMDA reaction. A single exception is MalC D165A, which produced mainly (+)-1, suggesting that Asp165 is required for reduction but not the IMDA reaction. The activity of MalC variants was also measured in assays with $\mathbf{1 1}$ (Fig. 5c). In agreement with the reconstitution assay, Asp108, Arg130 and Asp165 were required for reduction. Based on these data, we propose a reaction mechanism for MalC in which Arg130 serves as a proton donor potentially in conjunction with 2'-OH of NADPH ribose (Fig. 5d). NADPH is the hydride donor, and Asp165 may stabilize the positive charge of the zwitterionic substrate $\mathbf{1 1}$ and facilitate formation of the reactive azadiene 
intermediate 12. Stereocontrol of the IMDA reaction is primarily driven by shape complementarity, with contacts between substrate and Trp168 and the cofactor playing a critical role in the IMDA process.

a

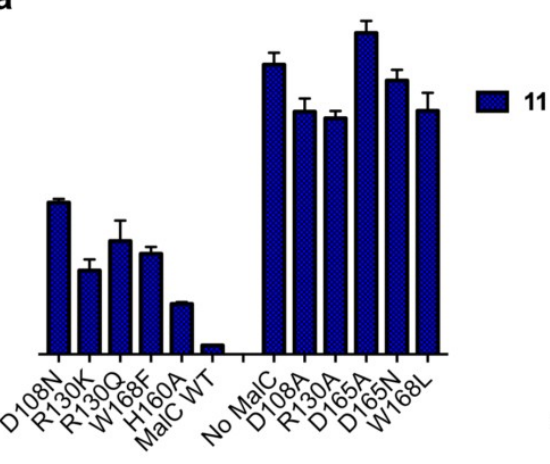

b

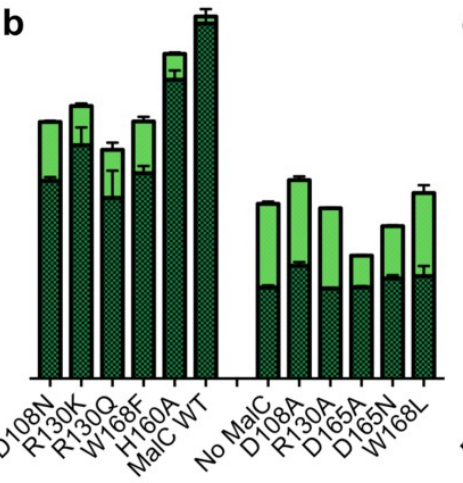

C

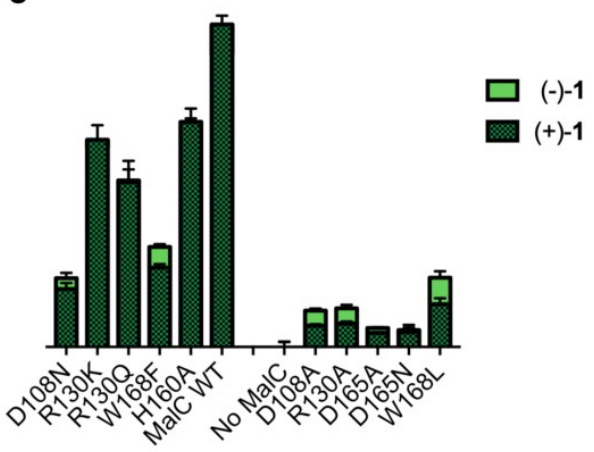

d

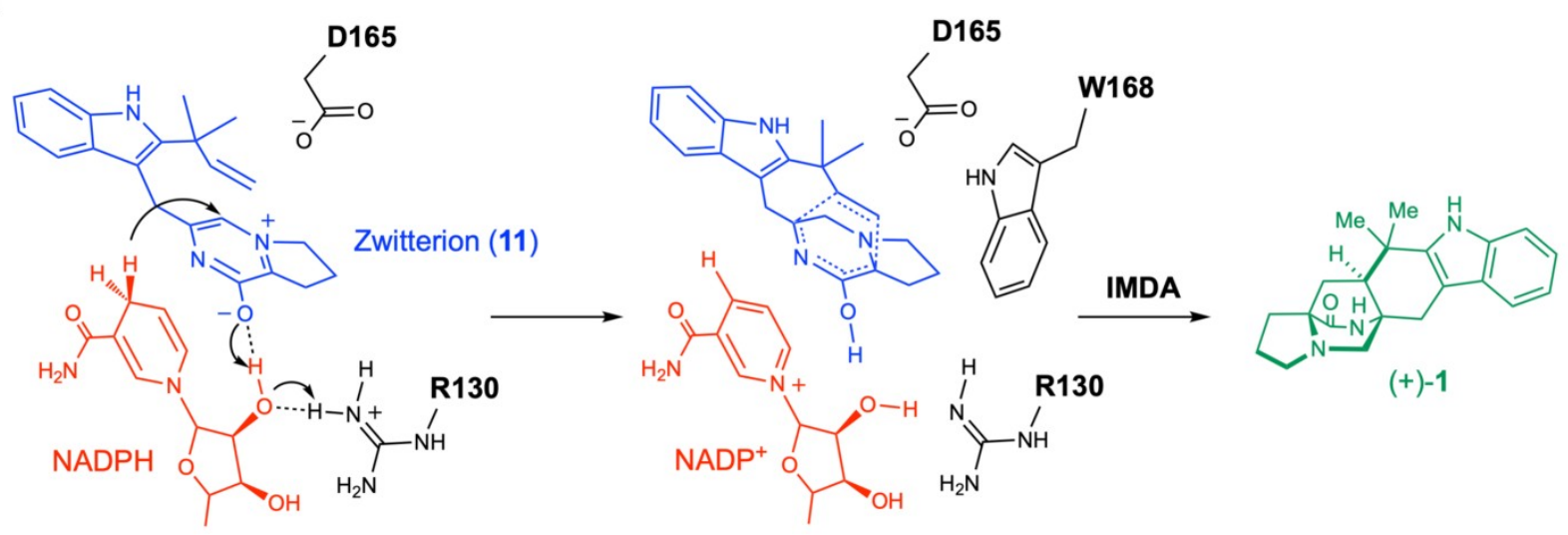

Figure 5. Catalytic mechanism of the MalC/PhqE-catalyzed Diels-Alder reaction.

$\mathrm{a}-\mathrm{b}$. Product profiles of 11 (blue), (+)-1 (dark green) and (-)-1 (light green) in the "MalG+MalE+MalC" reconstitution assay with MalC variants. c. MalC mutagenesis assessed by conversion of synthetic $\mathbf{1 1}$ to $(+)-1$. The results agree with those of the reconstitution assay in panel $b$. Product levels due to non-enzymatic conversion by NADPH were subtracted in all cases. All data represent the average of triplicate measurements (error bars, SD; $n=3$ ). d. Proposed catalytic mechanism for MalC/PhqE, with residue numbers for MalC (PhqE residue number $=$ MalC residue number +1$)$. Arg130 is the indirect proton donor, possibly mediated via the 2'-OH of NADPH ribose. Asp165 stabilizes the positive charge of 11, and hydride transfer from NADPH completes the first reduction step, forming an unstable azadiene intermediate. The subsequent IMDA reaction is accelerated primarily via entropy trapping, with diastereo- and enantioselectivity achieved via close packing of the $\mathrm{NADP}^{+}$nicotinamide, the azadiene and MalC Trp168, which together restrain the conformations of both the diene ring and the dienophile to ensure a single cycloaddition mode. 
The MalC/PhqE Diels-Alderases clearly evolved from an ancestral SDR (Fig. S24). The SDR catalytic Tyr and Lys were replaced by shorter, non-polar residues (Ile and Cys) providing space to accommodate the substrate. The "PDPGW" motif positions the essential Asp $3.0 \AA$ closer to the substrate compared to the corresponding amino acid in canonical SDRs. The SDR hydrogen bonding network is partially maintained since the catalytic Arg side chain of $\mathrm{MalC} / \mathrm{PhqE}$ occupies the position of the SDR catalytic Lys, providing a compelling example of protein evolution in molecular detail.

\section{Conclusions}

Our comprehensive approach to studying the Diels-Alder mediated construction of bicyclo[2.2.2]diazaoctane indole alkaloids represents a culmination of conceptual, experimental and computational studies initiated almost a half-century ago by $\operatorname{Birch}^{35}$. The divergent biogenesis to create the monooxo and dioxopiperazine-type molecules employed by diverse fungi was revealed through characterization of the respective biosynthetic gene clusters, which suggested a differential release mechanism from the functionally related bimodular NPRS systems (Fig. S1). This information was leveraged to design a biomimetic total synthesis of premalbrancheamide, providing a direct validation of the prenylated dipeptide azadiene intermediate and IMDA construction of the target natural product in racemic form. The basis for creating the $(+)$ antipodal form of premalbrancheamide via a presumed stereoselective DielsAlderase motivated our search for the corresponding enzyme from Mal and Phq pathways, resulting in the identification of a novel Diels-Alderase and a mechanistic understanding of enantio-induction. We have demonstrated that during biosynthetic assembly, the key step to produce the polycyclic core is catalyzed by a bifunctional reductase and intramolecular $[4+2]$ Diels-Alderase, MalC/PhqE, providing exquisite diastereo- and enantiocontrol. Derived from SDR ancestors, the active site of MalC/PhqE evolved to accommodate an aromatic zwitterion substrate, and both the reduction and the IMDA steps are NADP(H)-dependent. Our work reveals a distinct class of Diels-Alder enzymes and provides insights into the nature of IMDA catalysis. The availability of key intermediates provided from biomimetic synthesis enabled us to probe the molecular mechanism of this transformation in unprecedented detail. The MalC/PhqEcatalyzed reaction includes the remarkable step of "rescuing" an aromatic zwitterionic substrate $\mathbf{1 1}$ to create the bicyclic product $\mathbf{1 2}$ and avoid premature pathway termination. This brilliant evolutionary solution to protect the structural and sterochemical integrity of this architecturally unique family of alkaloids is, to the best of our knowledge, unprecedented and underscores the expanding plasticity and adaptability of secondary metabolite genes and enzymes. The Mal/Phqis biosynthetic sequence represents a novel "toolbox" for chemoenzymatic diversification of indole alkaloids with opportunities for facile access to improved calmodulin inhibitors, anthelmintics and other therapeutics to treat human and animal diseases. 
It is now recognized that fungi emerged very early in Earth's history, with current estimates reaching back as far as 2.4 billion years ago $^{36}$ and well before ocean oxygen concentrations increased during the Cambrian Explosion some 540 million years ago ${ }^{37}$. The conscription of the ancestral SDR family to function as both reductase and stereocontrolled cyclase suggests that these fungi evolved the ability to "rescue" the autoxidation of a productive azadiene species such as 12/9 (Fig. 1) from a non-productive shunt metabolite (11) that would have been obviated in a reducing environment. Our work suggests that such evolutionary processes where ancestral enzymatic functions are altered to adapt to environmental changes can overcome delimiting conditions of the organism, providing access to robust and diverse secondary metabolomes with likely selective advantages.

\section{References}

1 Finefield, J. M., Frisvad, J. C., Sherman, D. H. \& Williams, R. M. Fungal origins of the bicyclo[2.2.2]diazaoctane ring system of prenylated indole alkaloids. J Nat Prod 75, 812833, (2012).

2 Klas, K., Tsukamoto, S., Sherman, D. H. \& Williams, R. M. Natural Diels-Alderases: Elusive and Irresistable. J Org Chem 80, 11672-11685, (2015).

3 Klas, K. R. et al. Structural and stereochemical diversity in prenylated indole alkaloids containing the bicyclo[2.2.2] diazaoctane ring system from marine and terrestrial fungi. Nat Prod Rep 35, 532-558, (2018).

4 Robertson, A. P. et al. Paraherquamide and 2-deoxy-paraherquamide distinguish cholinergic receptor subtypes in ascaris muscle. J Pharmacol Exp Ther 303, 853-860, (2002).

5 Little, P. R. et al. Efficacy of a combined oral formulation of derquantel-abamectin against the adult and larval stages of nematodes in sheep, including anthelmintic-resistant strains. Vet Parasitol 181, 180-193, (2011).

6 Buxton, S. K. et al. Investigation of Acetylcholine Receptor Diversity in a Nematode Parasite Leads to Characterization of Tribendimidine-and Derquantel-Sensitive nAChRs. Plos Pathog 10, e1003870, (2014).

7 Mugishima, T. et al. Absolute stereochemistry of citrinadins A and B from marinederived fungus. J Org Chem 70, 9430-9435, (2005).

8 Mercado-Marin, E. V. et al. Total synthesis and isolation of citrinalin and cyclopiamine congeners. Nature 509, 318-324, (2014).

9 Porter, A. E. A. \& Sammes, P. G. A Diels-Alder Reaction of Possible Biosynthetic Importance. J Chem Soc Chem Comm, 1103, (1970).

10 Stocking, E. M. \& Williams, R. M. Chemistry and biology of biosynthetic Diels-Alder reactions. Angew Chem Int Ed Engl 42, 3078-3115, (2003).

$11 \mathrm{Li}, \mathrm{S}$. et al. Comparative analysis of the biosynthetic systems for fungal bicyclo[2.2.2] diazaoctane indole alkaloids: the $(+) /(-)$-notoamide, paraherquamide and malbrancheamide pathways. Medchemcomm 3, 987-996, (2012).

12 Stocking, E. M., Sanz-Cervera, J. F. \& Williams, R. M. Studies on the Biosynthesis of Paraherquamide: Synthesis and Incorporation of a Hexacyclic Indole Derivative as an Advanced Metabolite. Angew Chem Int Ed Engl 40, 1296-1298 (2001). 
13 Ding, Y. S. et al. Detection of VM55599 and preparaherquamide from Aspergillus japonicus and Penicillium fellutanum: Biosynthetic implications. Journal of Natural Products 71, 1574-1578, (2008).

14 Ding, Y. S., Greshock, T. J., Miller, K. A., Sherman, D. H. \& Williams, R. M. Premalbrancheamide: Synthesis, Isotopic Labeling, Biosynthetic Incorporation, and Detection in Cultures of Malbranchea aurantiaca. Org Lett 10, 4863-4866, (2008).

15 Ding, Y. et al. Genome-based characterization of two prenylation steps in the assembly of the stephacidin and notoamide anticancer agents in a marine-derived Aspergillus sp. $J$ Am Chem Soc 132, 12733-12740, (2010).

16 Martinez-Luis, S. et al. Malbrancheamide, a new calmodulin inhibitor from the fungus Malbranchea aurantiaca. Tetrahedron 62, 1817-1822, (2006).

17 Kim, H. J., Ruszczycky, M. W., Choi, S. H., Liu, Y. N. \& Liu, H. W. Enzyme-catalysed $[4+2]$ cycloaddition is a key step in the biosynthesis of spinosyn A. Nature 473, 109-112, (2011).

18 Hudson, G. A., Zhang, Z. G., Tietz, J. I., Mitchell, D. A. \& van der Donk, W. A. In Vitro Biosynthesis of the Core Scaffold of the Thiopeptide Thiomuracin. Journal of the American Chemical Society 137, 16012-16015, (2015).

19 Wever, W. J. et al. Chemoenzymatic Synthesis of Thiazolyl Peptide Natural Products Featuring an Enzyme-Catalyzed Formal [4+2] Cycloaddition. Journal of the American Chemical Society 137, 3494-3497, (2015).

20 Tian, Z. H. et al. An enzymatic [4+2] cyclization cascade creates the pentacyclic core of pyrroindomycins. Nat Chem Biol 11, 259-265, (2015).

21 Ohashi, M. et al. SAM-dependent enzyme-catalysed pericyclic reactions in natural product biosynthesis. Nature 549, 502-506, (2017).

$22 \mathrm{Li}$, L. et al. Genome Mining and Assembly-Line Biosynthesis of the UCS1025A Pyrrolizidinone Family of Fungal Alkaloids. J Am Chem Soc 140, 2067-2071, (2018).

23 Kato, N. et al. Control of the Stereochemical Course of [4+2] Cycloaddition during transDecalin Formation by Fsa2-Family Enzymes. Angew Chem Int Ed Engl 57, 9754-9758, (2018).

24 Fage, C. D. et al. The structure of SpnF, a standalone enzyme that catalyzes [4 + 2] cycloaddition. Nat Chem Biol 11, 256-258, (2015).

25 Zheng, Q. et al. Enzyme-Dependent [4 + 2] Cycloaddition Depends on Lid-like Interaction of the N-Terminal Sequence with the Catalytic Core in PyrI4. Cell Chem Biol 23, 352-360, (2016).

26 Byrne, M. J. et al. The Catalytic Mechanism of a Natural Diels-Alderase Revealed in Molecular Detail. J Am Chem Soc 138, 6095-6098, (2016).

27 Zheng, Q. F. et al. Structural Insights into a Flavin-Dependent [4+2] Cyclase that Catalyzes trans-Decalin Formation in Pyrroindomycin Biosynthesis. Cell Chemical Biology 25, 718-728, (2018).

28 Domingo, L. R., Zaragoza, R. J. \& Williams, R. M. Studies on the biosynthesis of paraherquamide A and VM99955. A theoretical study of intramolecular Diels-Alder cycloaddition. Journal of Organic Chemistry 68, 2895-2902, (2003).

29 Quadri, L. E. N. et al. Characterization of Sfp, a Bacillus subtilis phosphopantetheinyl transferase for peptidyl carrier protein domains in peptide synthetases. Biochemistry 37, 1585-1595, (1998). 
30 Nodvig, C. S., Nielsen, J. B., Kogle, M. E. \& Mortensen, U. H. A CRISPR-Cas9 System for Genetic Engineering of Filamentous Fungi. PLoS One 10, e0133085, (2015).

31 Fraley, A. E. et al. Function and Structure of MalA/MalA', Iterative Halogenases for Late-Stage C-H Functionalization of Indole Alkaloids. J Am Chem Soc 139, 1206012068, (2017).

32 Filling, C. et al. Critical residues for structure and catalysis in short-chain dehydrogenases/reductases. J Biol Chem 277, 25677-25684, (2002).

33 Oppermann, U. et al. Short-chain dehydrogenases/reductases (SDR): the 2002 update. Chem Biol Interact 143-144, 247-253 (2003).

34 Man, H. et al. Structures of Alcohol Dehydrogenases from Ralstonia and Sphingobium spp. Reveal the Molecular Basis for Their Recognition of 'Bulky-Bulky' Ketones. Top Catal 57, 356-365, (2014).

35 Birch, A. J. \& Wright, J. J. Studies in relation to biosynthesis. XLII. The structural elucidation and some aspects of the biosynthesis of the brevianamides-A and -E. Tetrahedron 26, 2329-2344 (1970).

36 Bengtson, S. et al. Fungus-like mycelial fossils in 2.4-billion-year-old vesicular basalt. Nat Ecol Evol 1, 141, (2017).

37 Fox, D. What sparked the Cambrian explosion? Nature 530, 268-270, (2016).

38 Barajas, J. F. et al. Comprehensive Structural and Biochemical Analysis of the Terminal Myxalamid Reductase Domain for the Engineered Production of Primary Alcohols. Chem Biol 22, 1018-1029, (2015).

39 Bonnett, S. A. et al. Structural and stereochemical analysis of a modular polyketide synthase ketoreductase domain required for the generation of a cis-alkene. Chem Biol 20, 772-783, (2013).

40 Chhabra, A. et al. Nonprocessive [2+2] e(-) off-loading reductase domains from mycobacterial nonribosomal peptide synthetases. P Natl Acad Sci USA 109, 5681-5686, (2012).

41 Wilson, D. J., Shi, C., Teitelbaum, A. M., Gulick, A. M. \& Aldrich, C. C. Characterization of AusA: A Dimodular Nonribosomal Peptide Synthetase Responsible for the Production of Aureusimine Pyrazinones. Biochemistry 52, 926-937, (2013).

42 Banani, H. et al. Genome sequencing and secondary metabolism of the postharvest pathogen Penicillium griseofulvum. BMC Genomics 17, 19, (2016).

\section{Data Availability}

Coordinates and associated structure factors have been deposited with the PDB under accession codes?.

Supplementary Information is available in the online version of the paper.

Acknowledgements This work was supported by the National Institutes of Health (R01 CA070375 to R.M.W. and D.H.S.), R35 GM118101, the Hans W. Vahlteich Professorship (to D.H.S.), and R01 DK042303 and the Margaret J. Hunter Professorship (to J.L.S.). GM/CA@APS is supported by the National Institutes of Health, National Institute of General 
Medical Sciences (AGM-12006) and National Cancer Institute (ACB-12002). The Advanced Photon Source is a U.S. Department of Energy (DOE) Office of Science User Facility operated by Argonne National Laboratory under Contract No. DE-AC02- 06CH11357. We thank Prof. Stephen Ragsdale for his assistance with anaerobic enzyme assays.

Author Contributions Q.D., S.A.N., J.L.S., R.M.W. and D.H.S. contributed to the experimental design. Q.D., S.A.N., A.E.F. and W.C.B. performed molecular cloning, protein over-expression and purification. Q.D., S.A.N. and A.E.F. performed all enzymatic assays and LC/MS analysis. S.A.N. and Q.D. carried out all crystallographic experiments, structural analysis and structurebased site-directed mutagenesis. J.D.S., K.R.K., J.M.F., A.D.S., T.J.M., L.Z., S.A.N. and V.V.S. synthesized and validated all compounds described in this study. Y.Y. and F.Y. carried out the genetic knockout experiment, F.Y. and Q.D. performed genetic annotation. J.N.S. and S.A.N. performed MD simulations. R.S.P. performed DFT calculations. Q.D., S.A.N., K.N.H., J.L.S., R.M.W. and D.H.S. evaluated the data and prepared the manuscript.

Author Information The authors declare no competing financial interests. Readers are welcome to comment on the online version of the paper. Correspondence and requests for materials should be addressed to D.H.S. (davidhs@umich.edu) and R.M.W. (robert.williams@colostate.edu). 


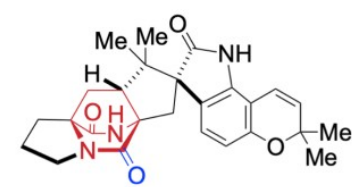

(-)-notoamide B Aspergillus protuberus

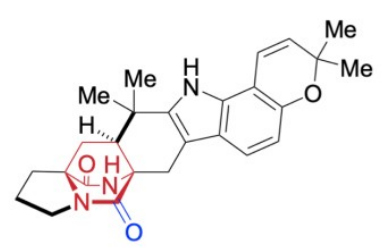

(+)-stephacidin A<smiles>[Y7]c1ccc2c(C[C@@H]3CC(=O)N4CCC[C@H]4C(=O)N3)c(C(C)(C)C=C)[nH]c2c1[Y2]</smiles>
Aspergillus ochraceus

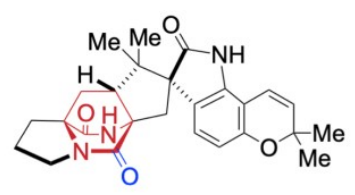
(+)-versicolamide B
Aspergillus amoenus

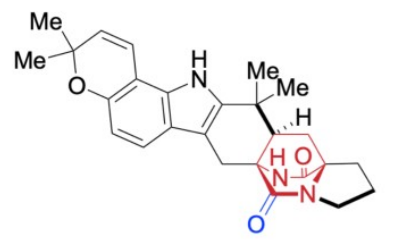

(-)-stephacidin A Aspergillus versicolor

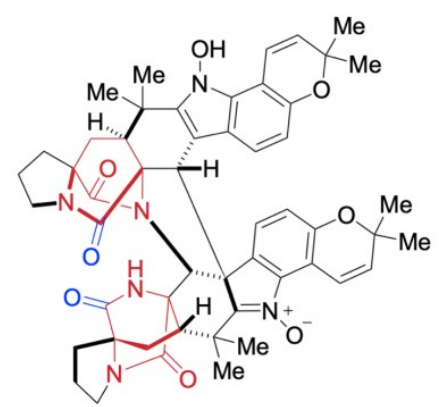

(-)-stephacidin B Aspergillus ochraceus

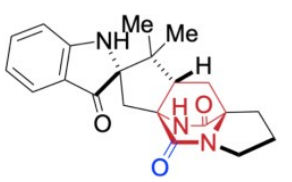

(+)-brevianamide A Penicillium brevicompactum

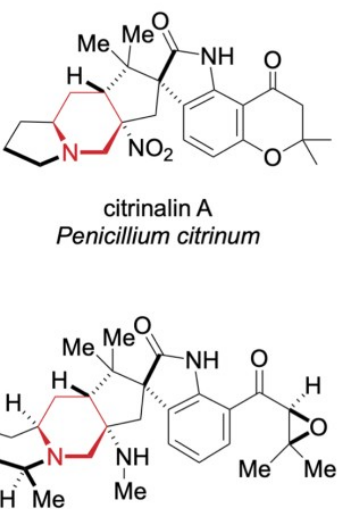

citrinadin B Penicillium citrinum

Supplementary Figure 1. Unified biogenesis of the dioxopiperazine and monooxopiperazine families of alkaloids possessing the bicyclo[2.2.2] diazaoctane core structures. The biocyclo[2.2.2]diazaoctane group is colored in red. The extra ketone group of dioxopiperazines are highlighted in blue. 
<smiles>C=CC(C)(C)c1[nH]c2cc(Cl)c(Cl)cc2c1C[C@H](N)C(=O)OCC</smiles>

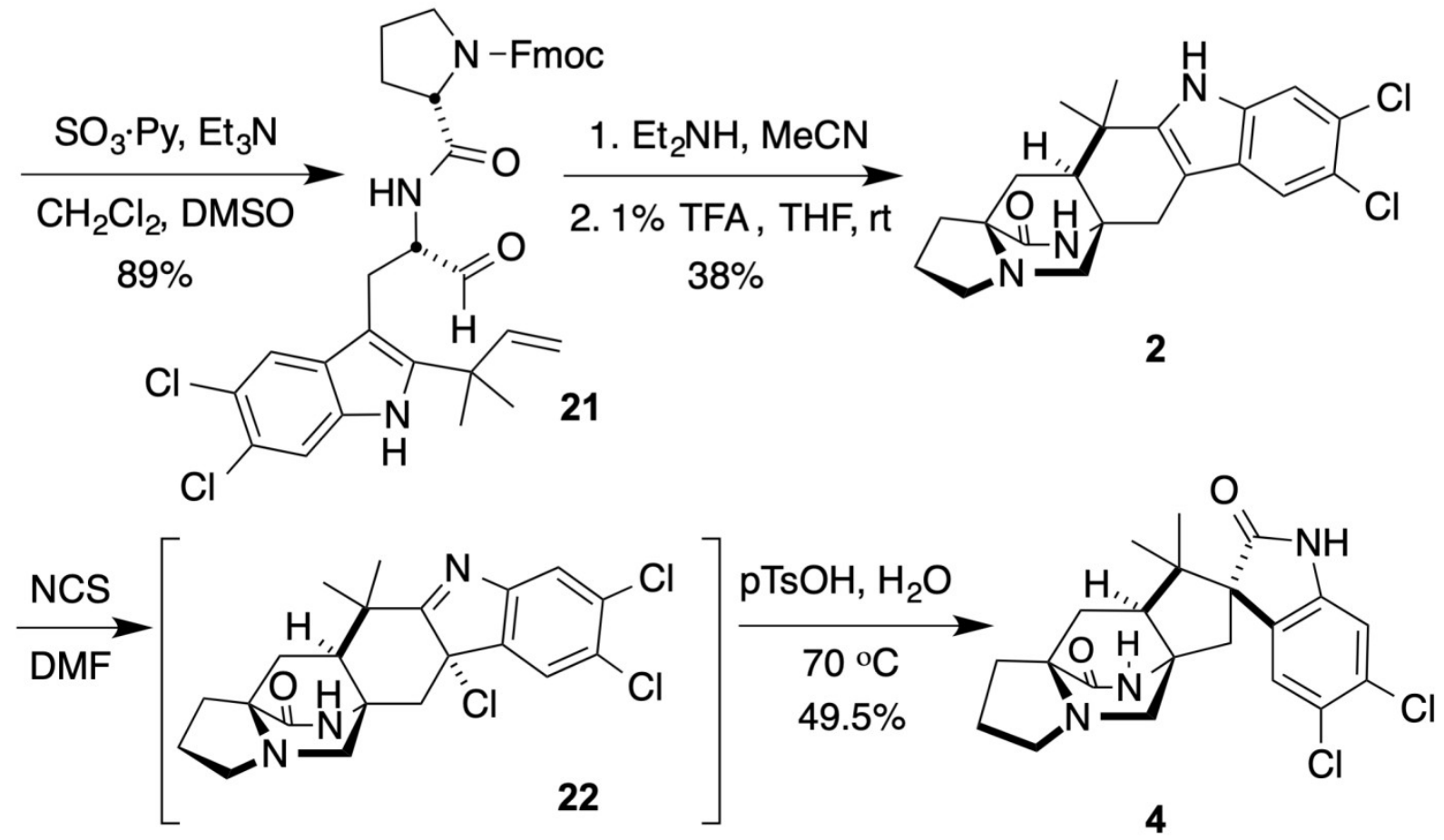

Supplementary Figure 2. Biomimetic synthesis of racemic malbrancheamide and spiromalbramide. We have applied an analogous strategy to two additional natural products, racemic ( \pm )-malbrancheamide (2) and ( \pm )-spiromalbramide (4) that underscores the utility of this new biomimetic paradigm. The key halogenated Fmoc-protected amino aldehyde (21) was prepared by peptide coupling of the reverse prenylated tryptophan methyl ester (18) with Fmocprotected proline amino acid (14) using HATU (87\% yield). The ethyl ester was reduced with sodium borohydride $(\mathbf{2 0} ; \mathbf{9 0 \%})$ and followed by a Doering-LaFlamme oxidation to provide the $\mathrm{N}-\mathrm{Fmoc}$ aldehyde $\mathbf{2 1}$ in $89 \%$ yield. The Fmoc group was removed with diethylamine, and the crude product was directly treated with a degassed solution of $1 \%$ TFA in THF at room temperature to provide malbrancheamide (racemic) $\mathbf{2}$ in $38 \%$ yield. $\mathbf{2}$ was treated with $\mathrm{N}$ chlorosuccinimide to form the incipient chloroindoline intermediate (22), which was directly 
hydrated under acidic conditions to undergo a pinacol-type rearrangement and form spiromalbramide (racemic) 4 in $49.5 \%$ yield. Including the four steps required to synthesize the reverse-prenylated tryptophan species 18, the synthesis of malbrancheamide was achieved in eight steps from commercially available materials and only four steps in the longest linear sequence; one additional transformation (two steps, one operation) being required to reach spiromalbramide. 

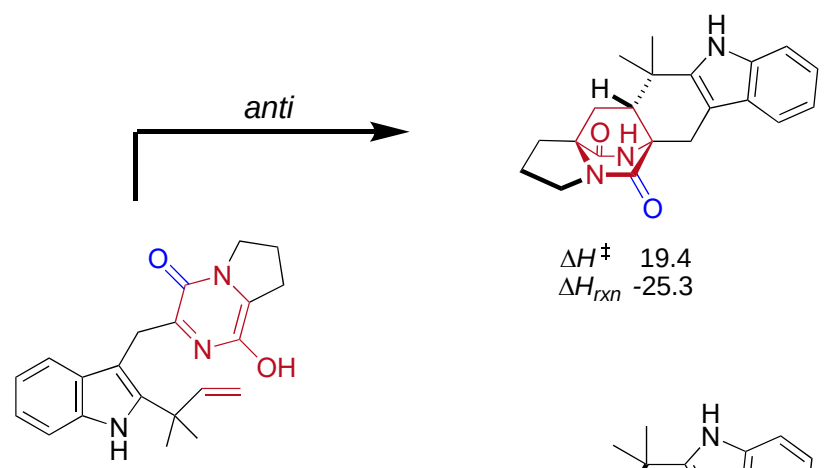

$$
\begin{array}{lr}
\Delta H^{\ddagger} & 19.4 \\
\Delta H_{r \times n} & -25.3
\end{array}
$$
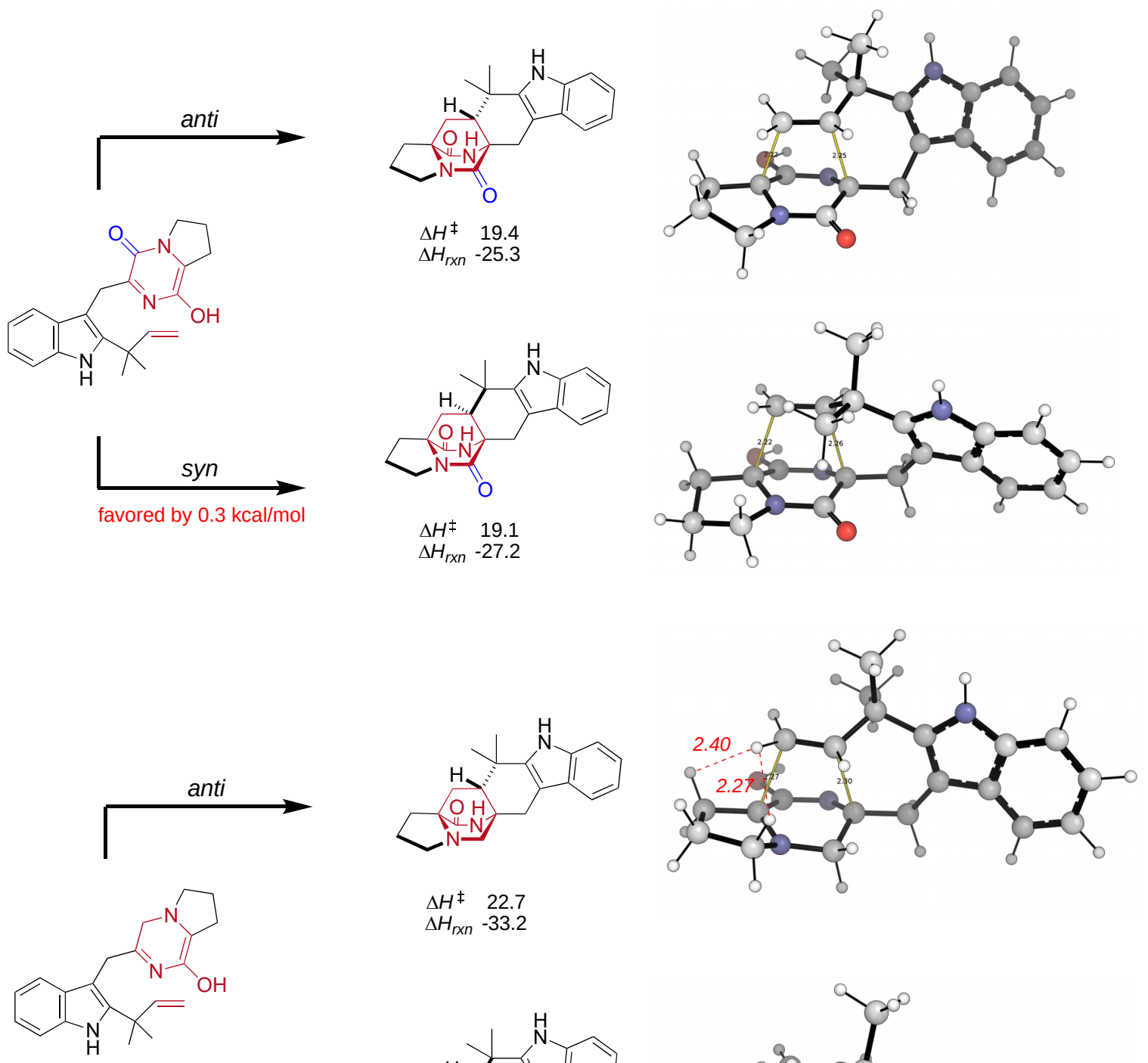

$$
\begin{array}{lr}
\Delta H^{\ddagger} & 22.7 \\
\Delta H_{r \times n} & -33.2
\end{array}
$$
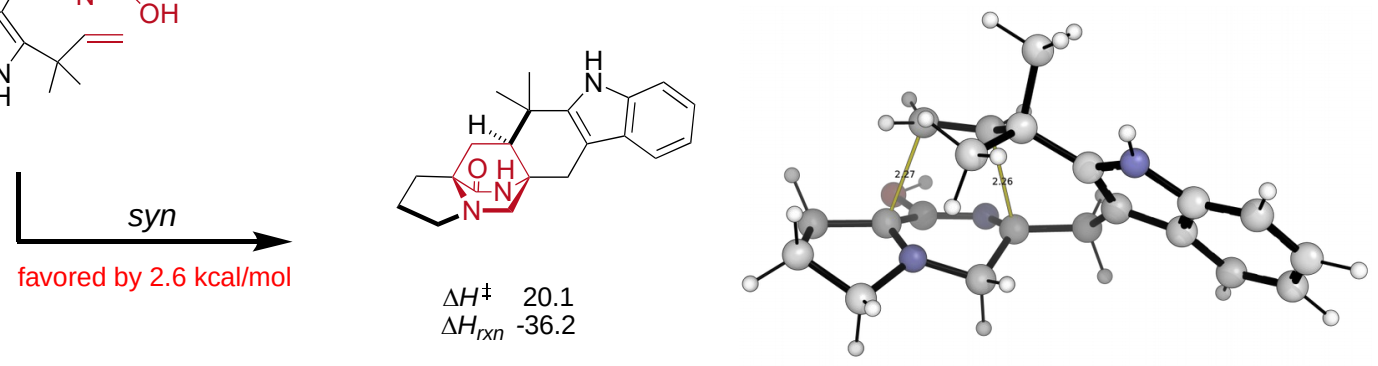

Supplementary Figure 3. M062X-D3/6-31+G(d,p) intramolecular Diels-Alder transition structures (TSs) for both oxidation states represented by azadiene, predict a relatively modest syn-: anti-diastereoselectivity for the more oxygenated azadiene species of $0.3 \mathrm{kcal} / \mathrm{mol}^{28}$. This value has been corroborated experimentally in several systems where the syn-: anti-ratio is typically around 2.5:1. The reduced azadiene specieshas a more substantial TS difference of 2.6 $\mathrm{kcal} / \mathrm{mol}$ favoring the syn-cycloadduct. The pyrrolidine ring adopts different conformations in these syn- and anti- TSs, puckering towards the dienophile in the less-favorable structure and resulting in short $\mathrm{H}---\mathrm{H}$ contacts. Consistent with greater levels of selectivity, the only detectable diastereomeric cycloadducts were the syn-diastereomers by comparison with authentic, synthetic samples of the corresponding anti-diastereomers. 

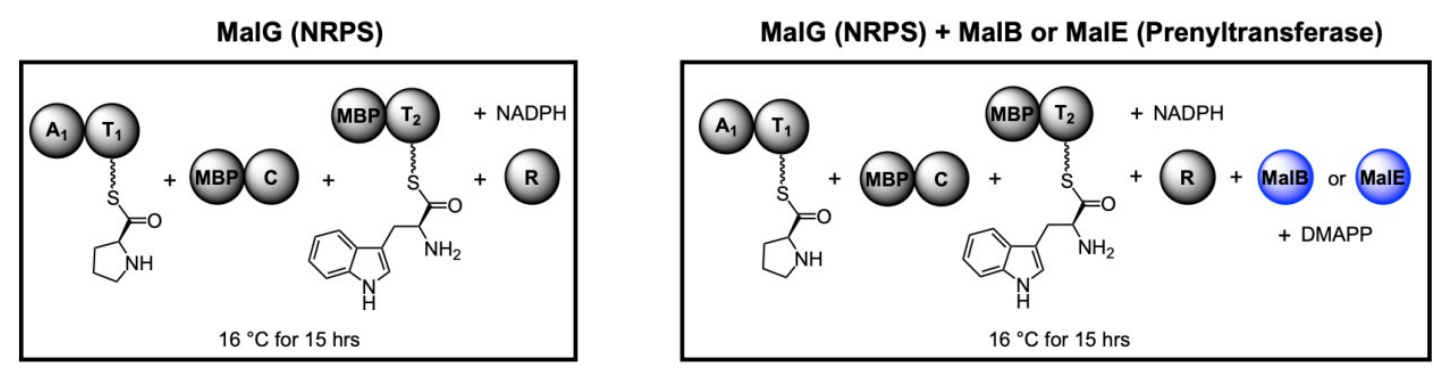

MalG + MalE + MalC (Diels-Alderase)
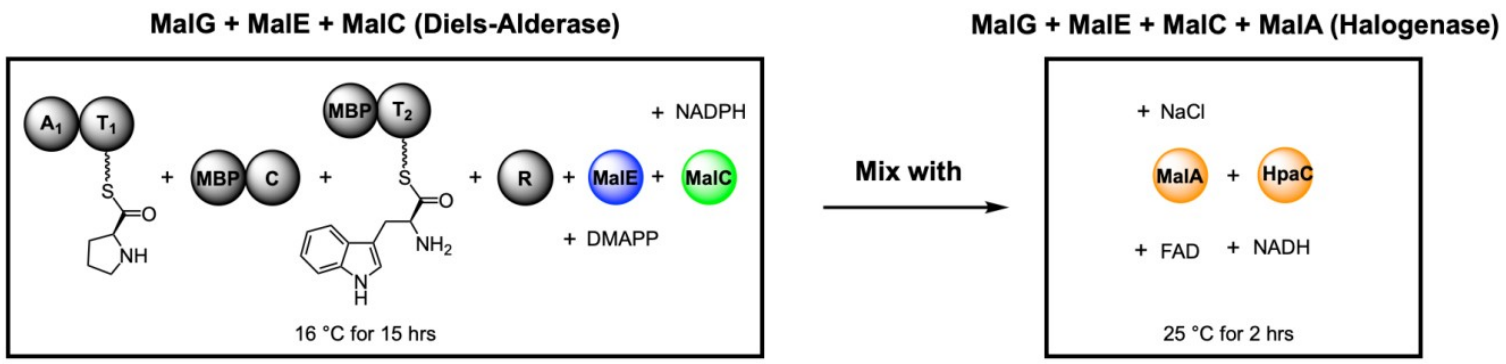

Supplementary Figure 4. Scheme of in vitro reconstitution assays. The flavin-dependent MalA halogenase requires a recycling system to reduce $\mathrm{FAD}$ to $\mathrm{FADH}_{2}$ after each catalytic cycle, here $\mathrm{HpaC}$ reductase $+\mathrm{NADH}^{31}$. MBP $=$ maltose binding protein. 
MalB
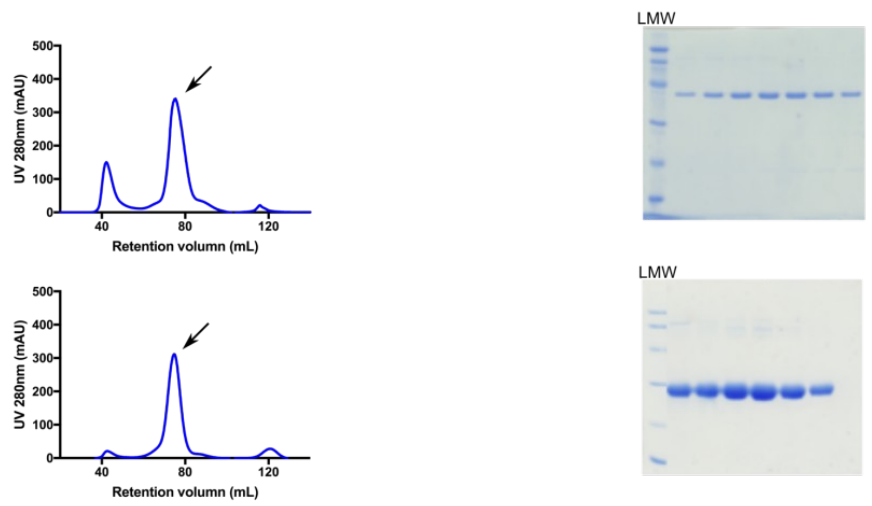

MalC
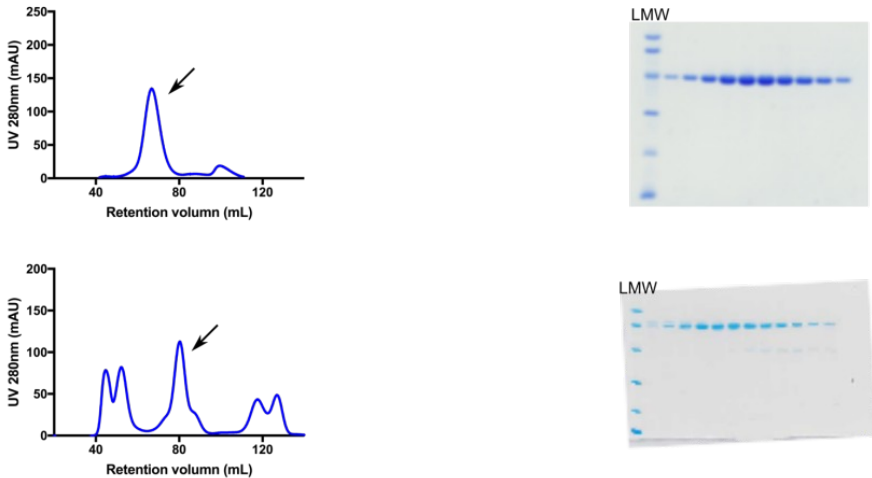

MalG C (MBP fusion)
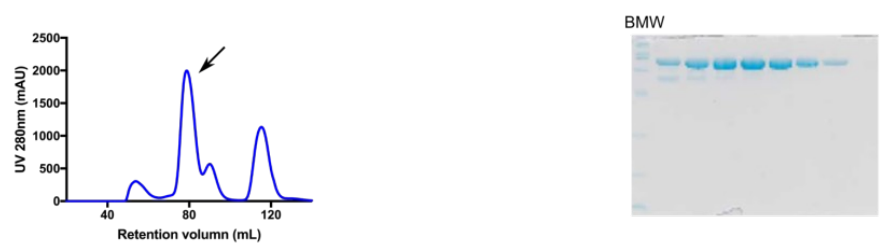

MalG $\mathrm{T}_{2}$ (MBP fusion)
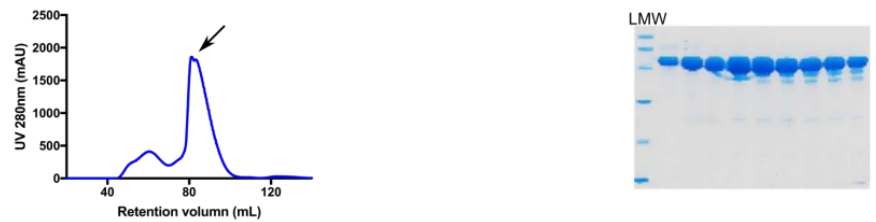

MalG R
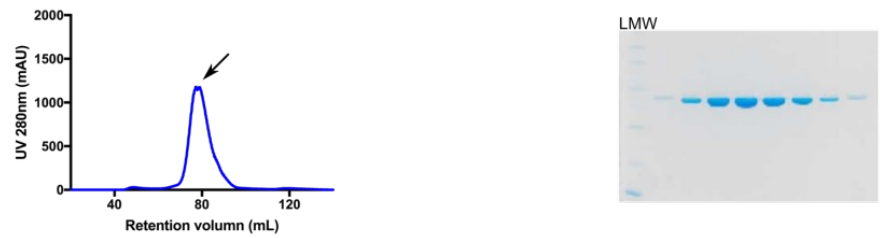

Supplementary Figure 5. Purification of enzymes and enzymatic domains involved in malbrancheamide biosynthesis with gel filtration profiles at left and SDS gels of the indicated peaks at right. Recombinant MalA was produced as previously described ${ }^{31}$. Protein molecular weight standards were LMW: 97.4, 66.2, 45.0, 31.0, 21.5, $14.4 \mathrm{kDa}$; and BMW: 200, 116.3, $97.4,66.2$, 45.0, 31.0, 21.5, 14.4, 6.5 kDa. 
a
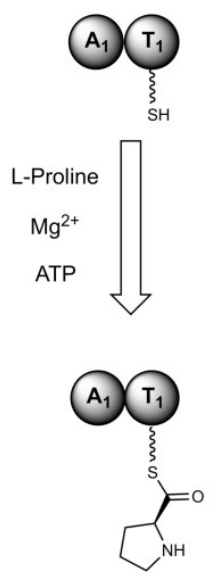

b
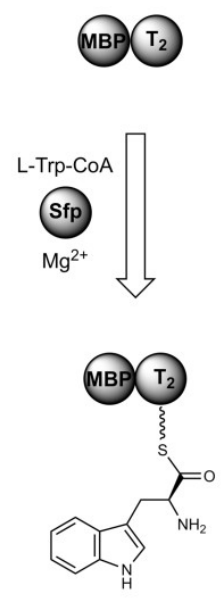

C<smiles>O=C(N[C@H](Cc1c[nH]c2ccccc12)C(=O)O)C1CCCO1</smiles>

23

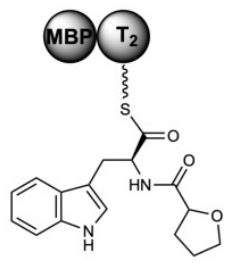

23-MBP-MalG $\mathrm{T}_{2}$

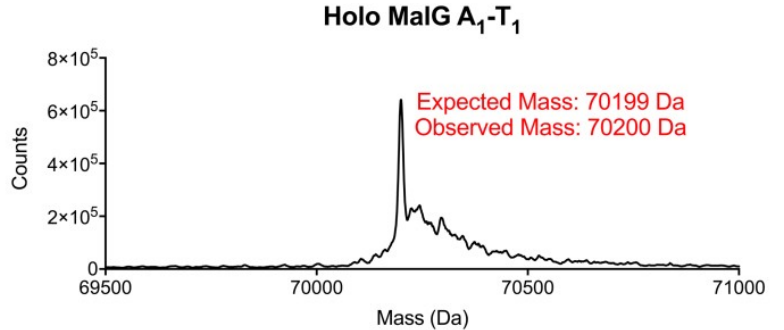

Pro-MalG A $\mathrm{A}_{1} \mathrm{~T}_{1}$

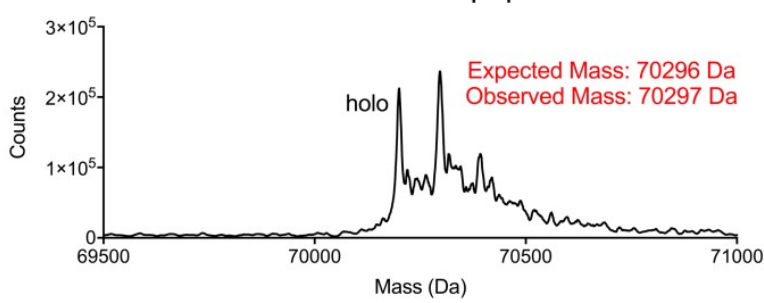

Apo MBP-MalG $\mathrm{T}_{2}$

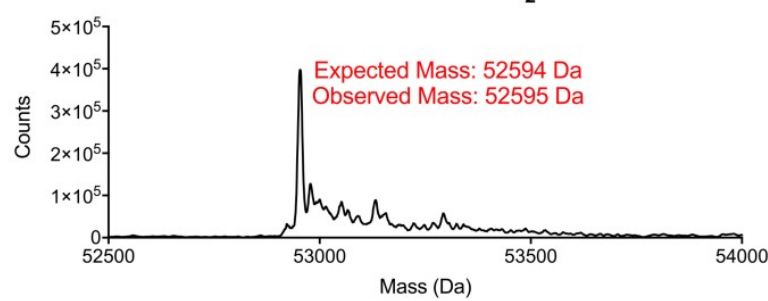

Trp-MBP-MalG T $\mathbf{T}_{2}$

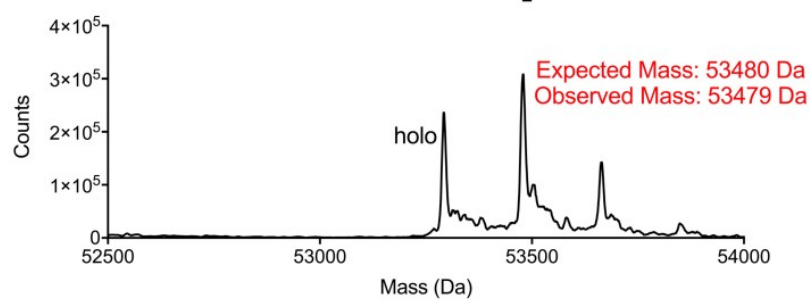

23-MBP-MalG T 2

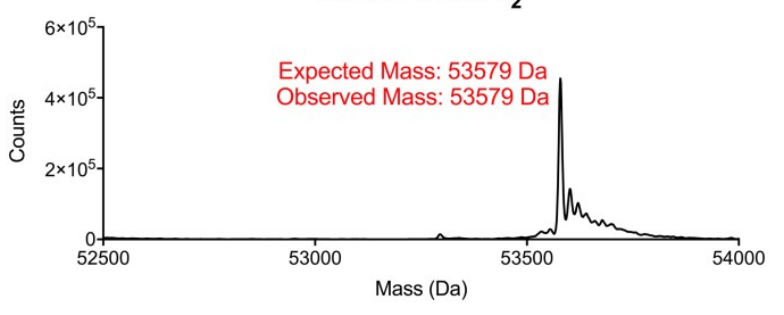


Supplementary Figure 6. Substrate loading of MalG $\mathrm{T}_{1}$ and $\mathrm{T}_{2}$ domains. Protein MS was applied to analyze efficiency of substrate loading, confirming successful loading in all cases.

a

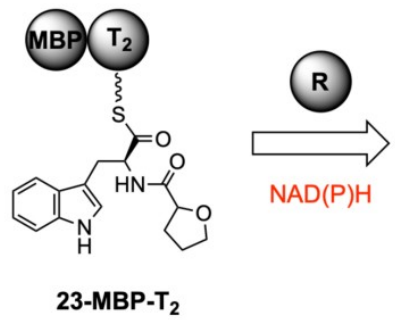

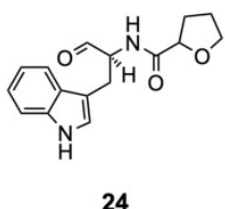

24
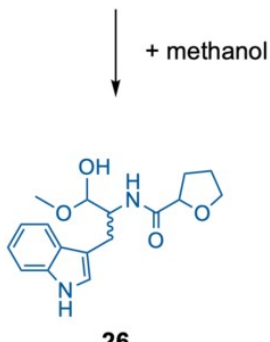

26

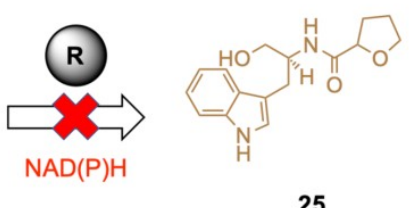

25

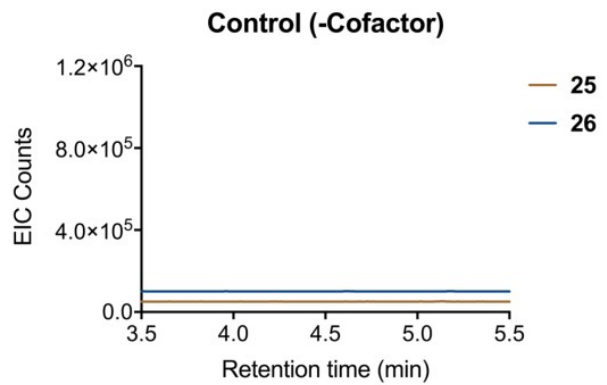

d

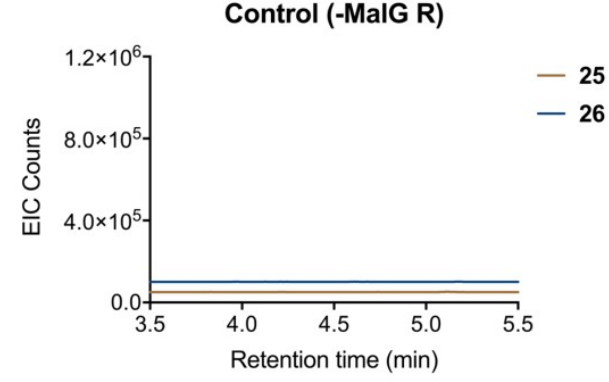

e

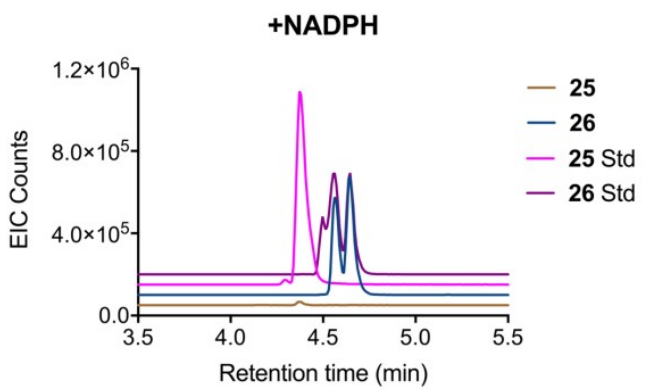

Supplementary Figure 7. MalG R catalyzes a 2-electron reductive release reaction. a. Reaction scheme of MalG R. b - c. EIC profiles of control experiments, with no enzyme (b) or no cofactor (c). $d-e$. EIC profiles of the MalG R-catalyzed reaction, using NADH (d) or NADPH (e) as cofactor. NADPH is the preferred cofactor. 25, the product of a 4-electron reduction was not detected. 

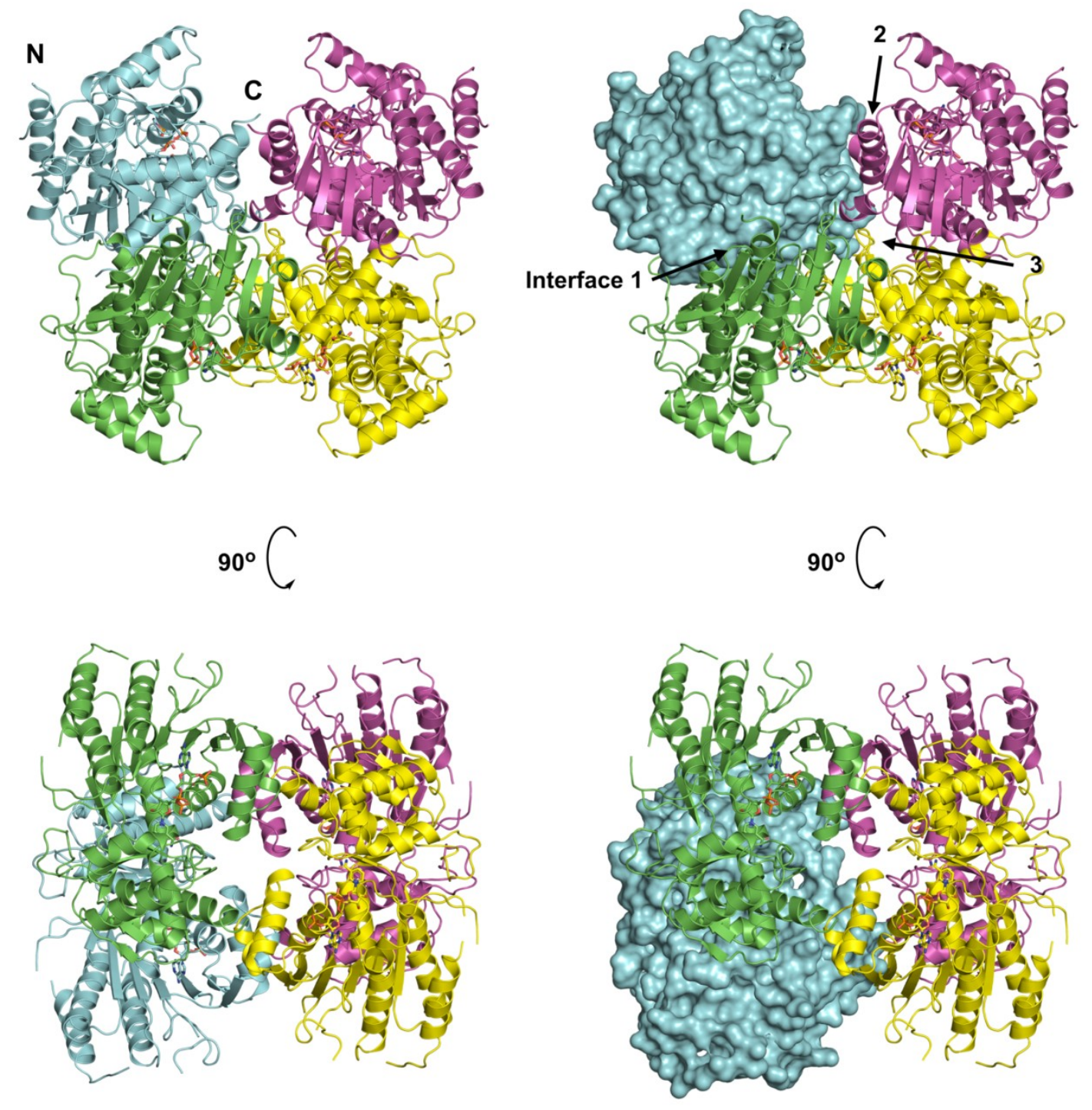

Supplementary Figure 8. Crystal structure of the PhqB R tetramer, with the four subunits shown in contrasting colors cartoon representation in (a), and the cyan subunit shown in surface representation in (b). The $\mathrm{N}$ - and $\mathrm{C}$-termini are marked for the cyan subunit. The excised PhqB R tetramer has D2 point symmetry, and each subunit contacts all three other subunits. The tetrameric oligomer state, apparently inherited from short-chain dehydrogenase/reductase (SDR) ancestors, differs from the generally monomeric NRPS situation. However, four N-termini that link to the rest of MalG are at the exterior of the tetramer and well separated from one another in an arrangement that would allow flexible tethering of a "monomeric" NRPS module. 

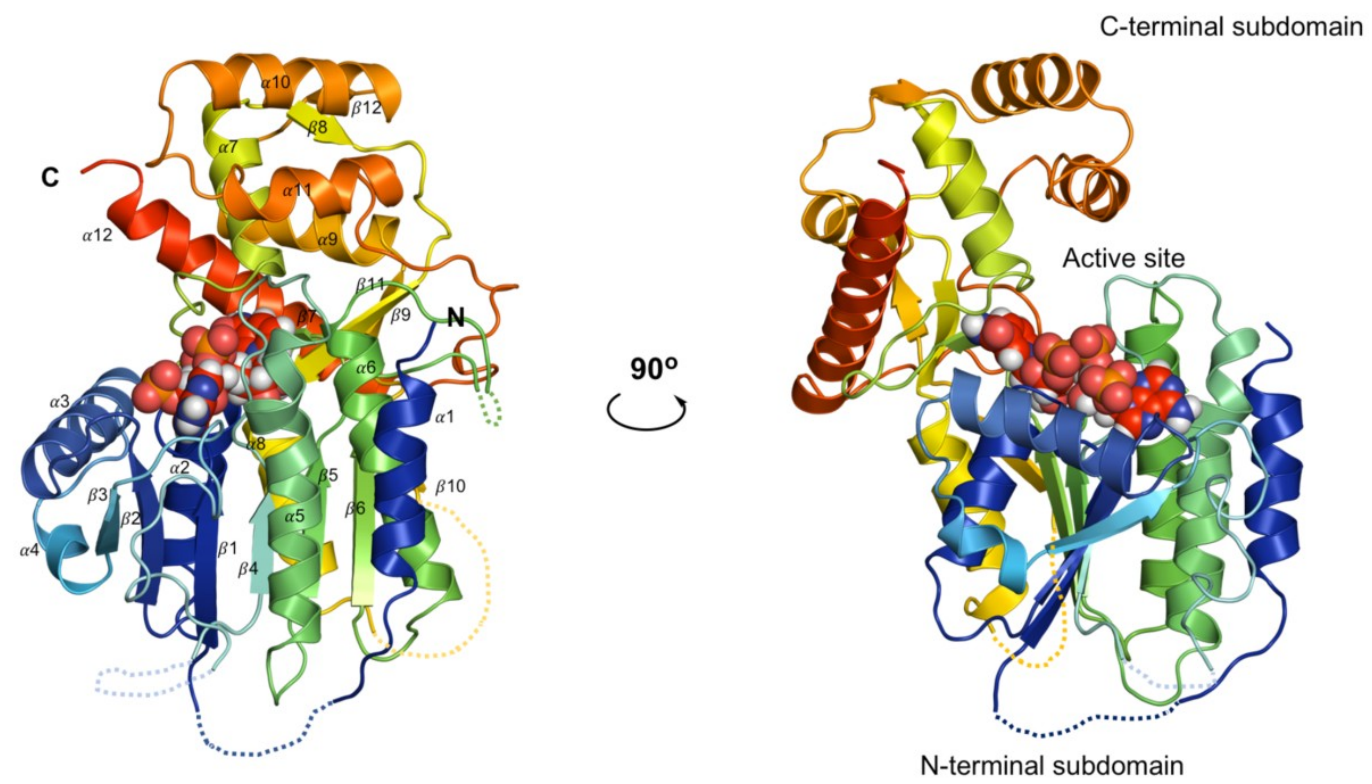

Supplementary Figure 9. Structure of the PhqB R subunit colored as a rainbow from blue Nterminus to red $\mathrm{C}$-terminus. PhqB $\mathrm{R}$ consists of an $\mathrm{N}$-terminal nucleotide-binding subdomain and a C-terminal substrate-binding subdomain, which recognizes Pro-Trp- $\mathrm{T}_{2}$. The nucleotide-binding subdomain has a typical Rossmann fold, with a parallel $\beta$ sheet $(\beta 1, \beta 2, \beta 3, \beta 4, \beta 5, \beta 6$ and $\beta 10)$ flanked by six $\alpha$ helices $(\alpha 2, \alpha 3, \alpha 4, \alpha 5, \alpha 6$ and $\alpha 8)$ and an invariant "TGX ${ }_{3}$ GXG" motif (Ploop), as well as conserved Arg2070 and Arg2080, which coordinate the adenosine 2'-phosphate and account for the selectivity of NADPH (shown as spheres) over NADH. The C-terminal subdomain covers the active site, is unique to NRPS terminal reductases, and is composed of five $\alpha$ helices $(\alpha 7, \alpha 9, \alpha 10, \alpha 11$ and $\alpha 12)$. 

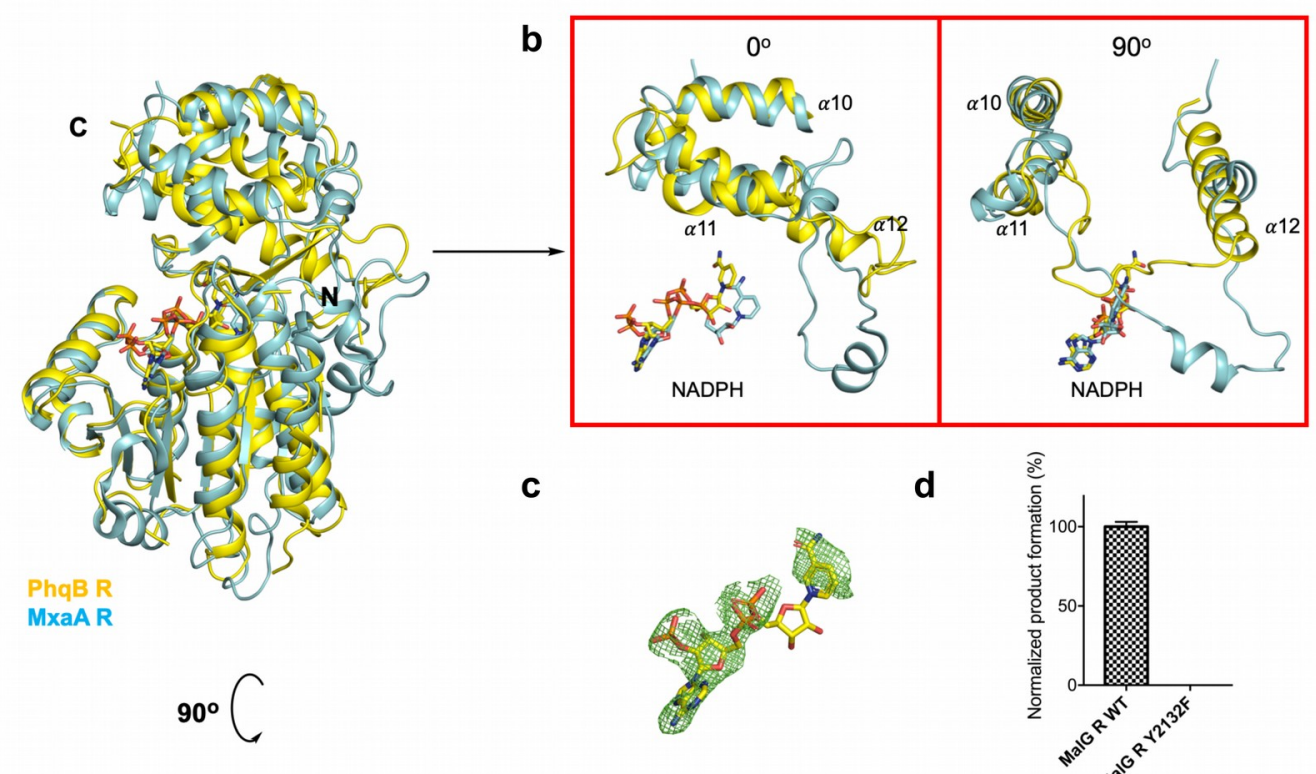

C
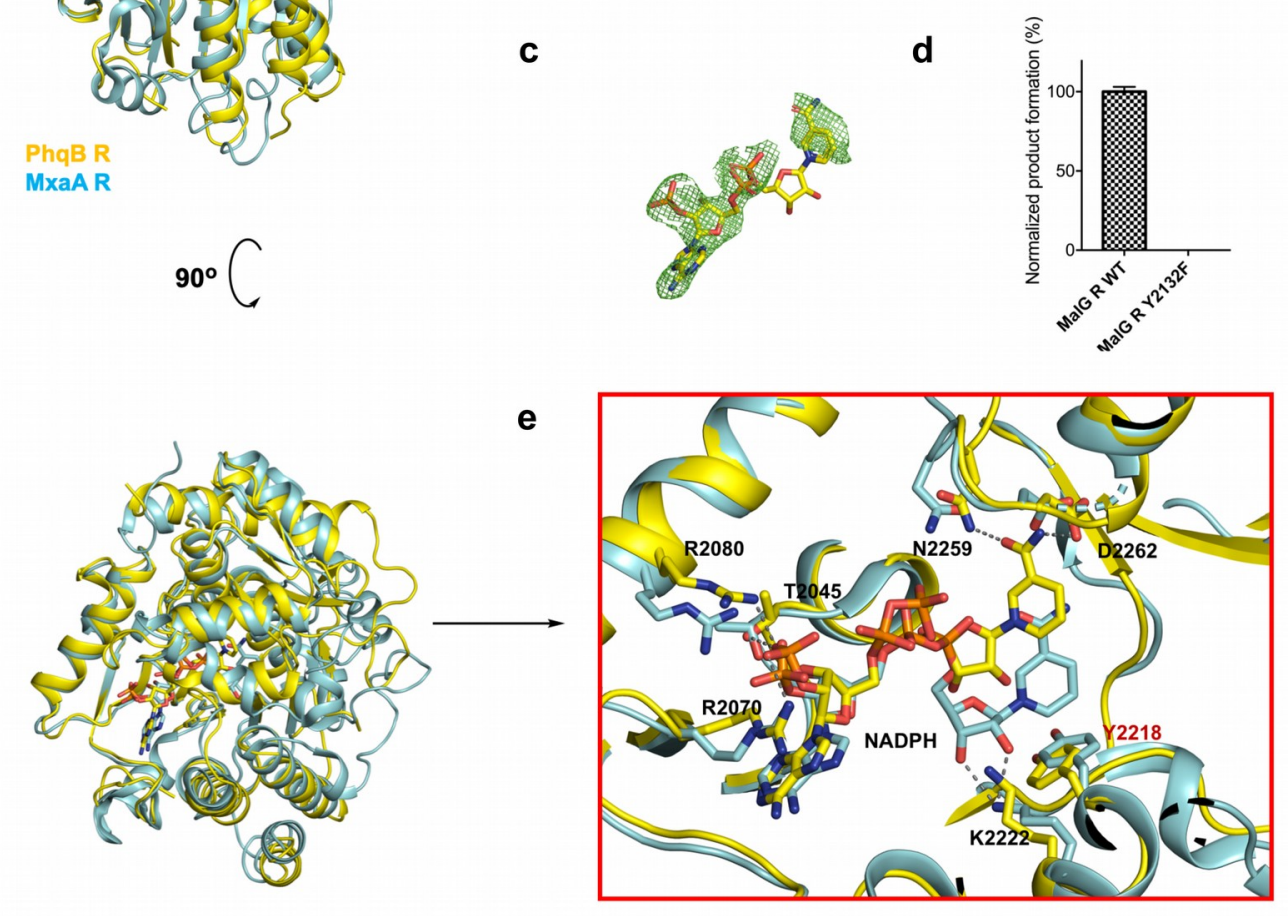

Supplementary Figure 10. Comparison of fungal and bacterial NRPS R domains. a. Superposition of the PhqB R subunit (yellow) and the bacterial MxaA R ${ }^{38}$ (cyan, PDB ID: 4U7W). b. The major structural difference lies in the C-terminal subdomain. Relative to bacterial NRPS R domain structures, PhqB R $\alpha 12$ is tilted towards the core with a significantly shorter preceding loop that lacks a short helix. In the PhqB R tetramer this is the site of a subunit contact, which does not exist in the monomeric bacterial $\mathrm{R}$ domain. c. Electron density map for the PhqB NADPH cofactor $\left(\mathrm{F}_{\mathrm{o}}-\mathrm{F}_{\mathrm{c}}\right.$ omit contoured at $\left.3 \sigma\right)$. In the co-crystal structure of PhqB R and NADPH, the nicotinamide ring of NADPH is poorly resolved and partially occupies a noncatalytic position. This is in contrast to bacterial NRPS R domains and is correlated with strikingly different structures for $\alpha 11$-loop- $\alpha 12$ in the fungal and bacterial $\mathrm{R}$ domains. A poorly ordered nicotinamide also occurs in bacterial modular polyketide synthase (PKS) B-type ketoreductases $^{39}$. Substrate may be required for optimal cofactor binding d. Lack of detectable activity in MalG R/Y2132F (error bars, SD; $n=3$ ). e. Active site detail. The active site contains conserved residues Tyr2218 and Lys2222, suggestive of a shared reaction mechanism with bacterial NRPS terminal R domains ${ }^{38,40,41}$ and other Tyr-dependent SDRs ${ }^{32,33}$ in which a catalytic Tyr serves as a proton donor and a catalytic Lys facilitates proton transfer. The catalytic Tyr is 
labeled in red. In the PhqB R, the nicotinamide is in a non-catalytic position away from Tyr2218 and Lys2222, but is hydrogen bonded to conserved Asn2259 and Asp2262. 
a

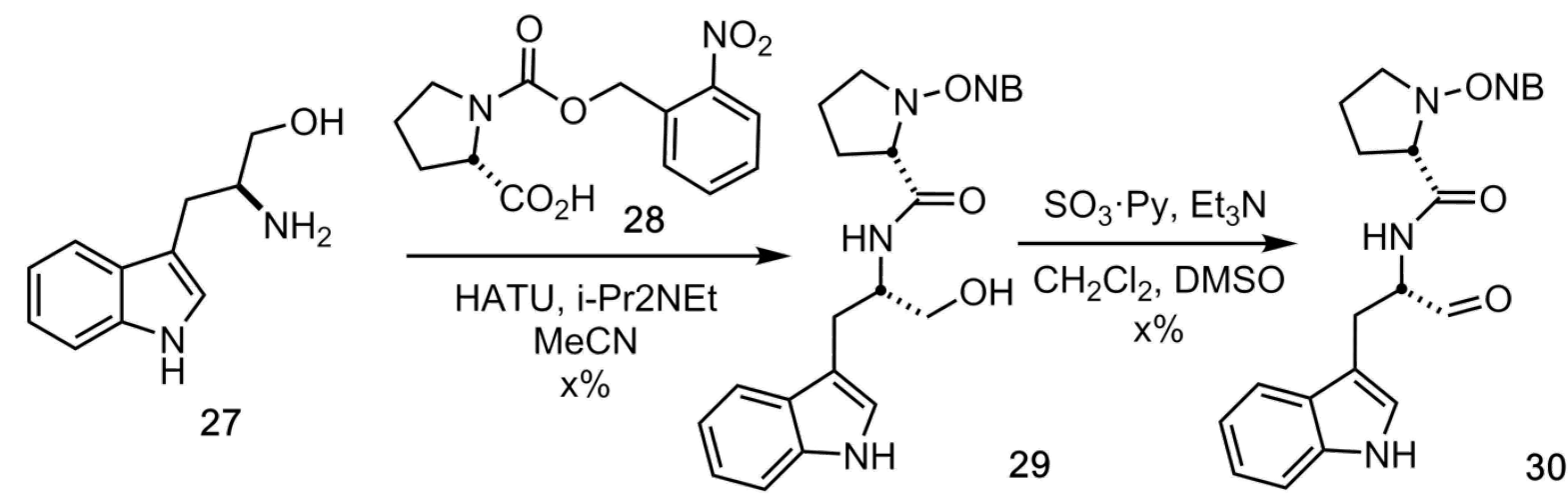

b<smiles>C=CC(C)(C)c1[nH]c2ccccc2c1CC(N)CO</smiles>

Supplementary Figure 11. Synthetic scheme for the ONB protected dipeptide aldehyde 30 (a) and ONB protected prenyl dipeptide 33 (b). 
Anaerobic
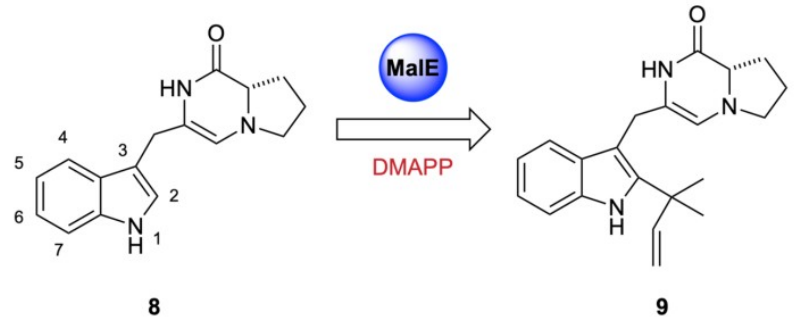

\section{$\downarrow$}

spontaneous oxidation

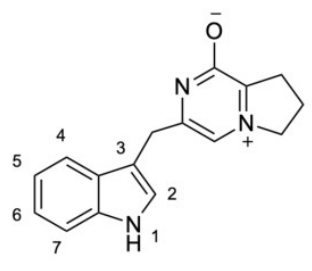

10

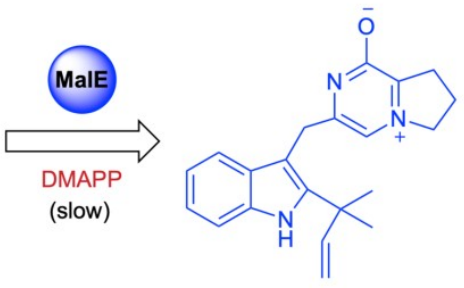

11

d

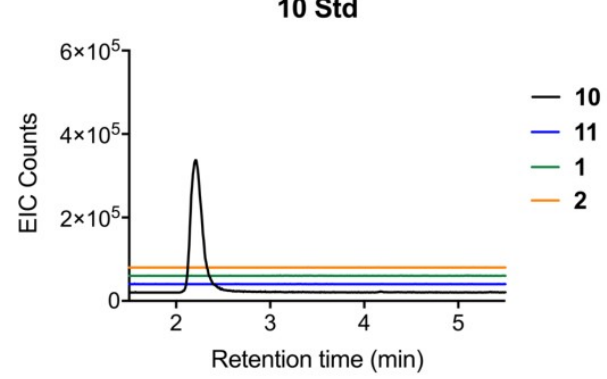

e

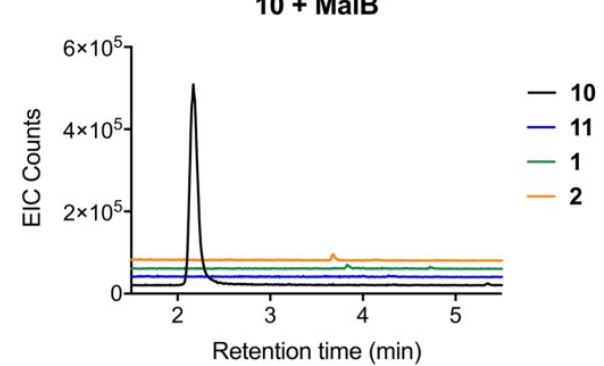

C

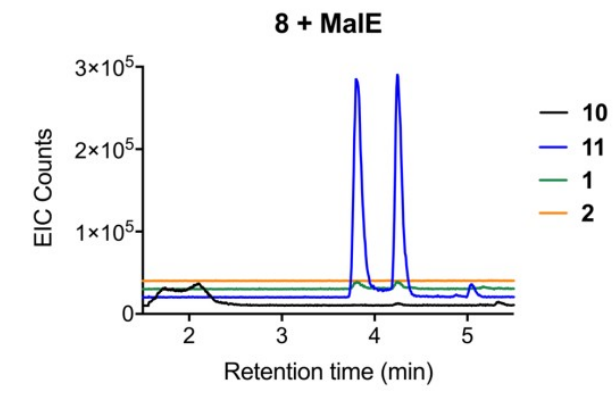

$10+$ MalE

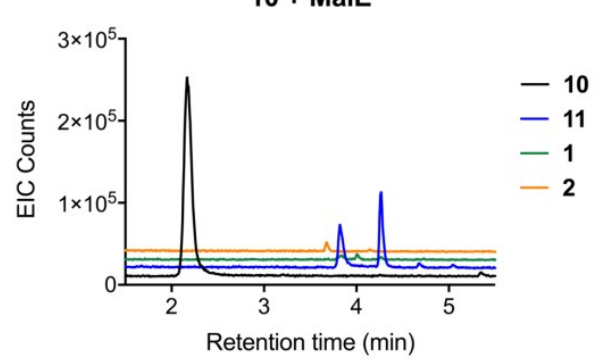

Supplementary Figure 12. Prenylation of $\mathbf{8}$ (anaerobic) and $\mathbf{1 0}$ (aerobic). 8 is the natural substrate of MalE. 10 can be prenylated by MalE but not MalB. a. C2 reverse prenyltransfer reaction scheme. b. EIC profile of $\mathbf{1 0}$ authentic standard. c. EIC profile of $\mathbf{8}$ prenylation by MalE in anaerobic conditions. Spontaneous oxidation of $\mathbf{9}$ to $\mathbf{1 1}$ occurred when the reaction mixture was subjected to LC/MS analysis. d. EIC profile of $\mathbf{1 0}$ prenylation by MalB in anaerobic conditions. No prenylated product was detected. e. EIC profile of $\mathbf{1 0}$ prenylation by MalE, illustrating that oxidized $\mathbf{1 0}$ is less favored than $\mathbf{8}$. 

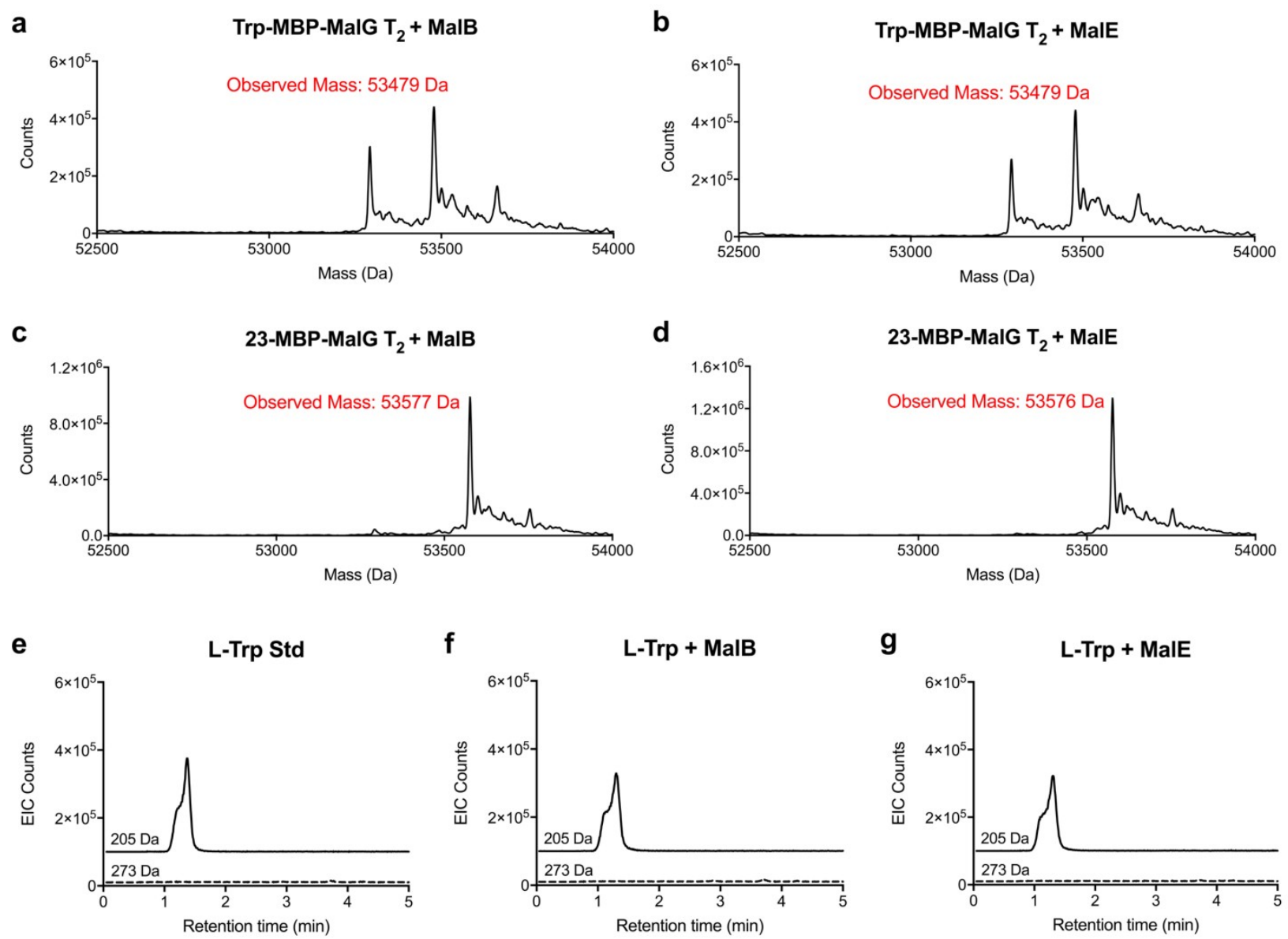

Supplementary Figure 13. Timing of prenylation reaction after NRPS reductive offloading. a. Protein MS profile of L-Trp-MBP-MalG $T_{2}$ after 2 hours of MalB and DMAPP incubation. $b$. Protein MS profile of L-Trp-MBP-MalG $\mathrm{T}_{2}$ after 2 hours of MalE and DMAPP incubation. c. Protein MS profile of 23-MBP-MalG $\mathrm{T}_{2}$ after 2 hours of MalB and DMAPP incubation. $\mathrm{d}$. Protein MS profile of 23-MBP-MalG $\mathrm{T}_{2}$ after 2 hours of MalE and DMAPP incubation. In all cases, no protein mass change was observed, showing no prenylation. e. EIC profile of L-Trp authentic standard. f. EIC profile of L-Trp after 2 hours of MalB and DMAPP incubation. g. EIC profile of L-Trp after 2 hours of MalE and DMAPP incubation. In all cases, no prenylation was detected ( $\mathrm{L}-\operatorname{Trp} \mathrm{M}+\mathrm{H}^{+} m / z=205$; Prenylated L-Trp $\mathrm{M}+\mathrm{H}^{+} m / z=273$ ), demonstrating that MalG NRPS functions as the first enzyme in the malbrancheamide pathway. 


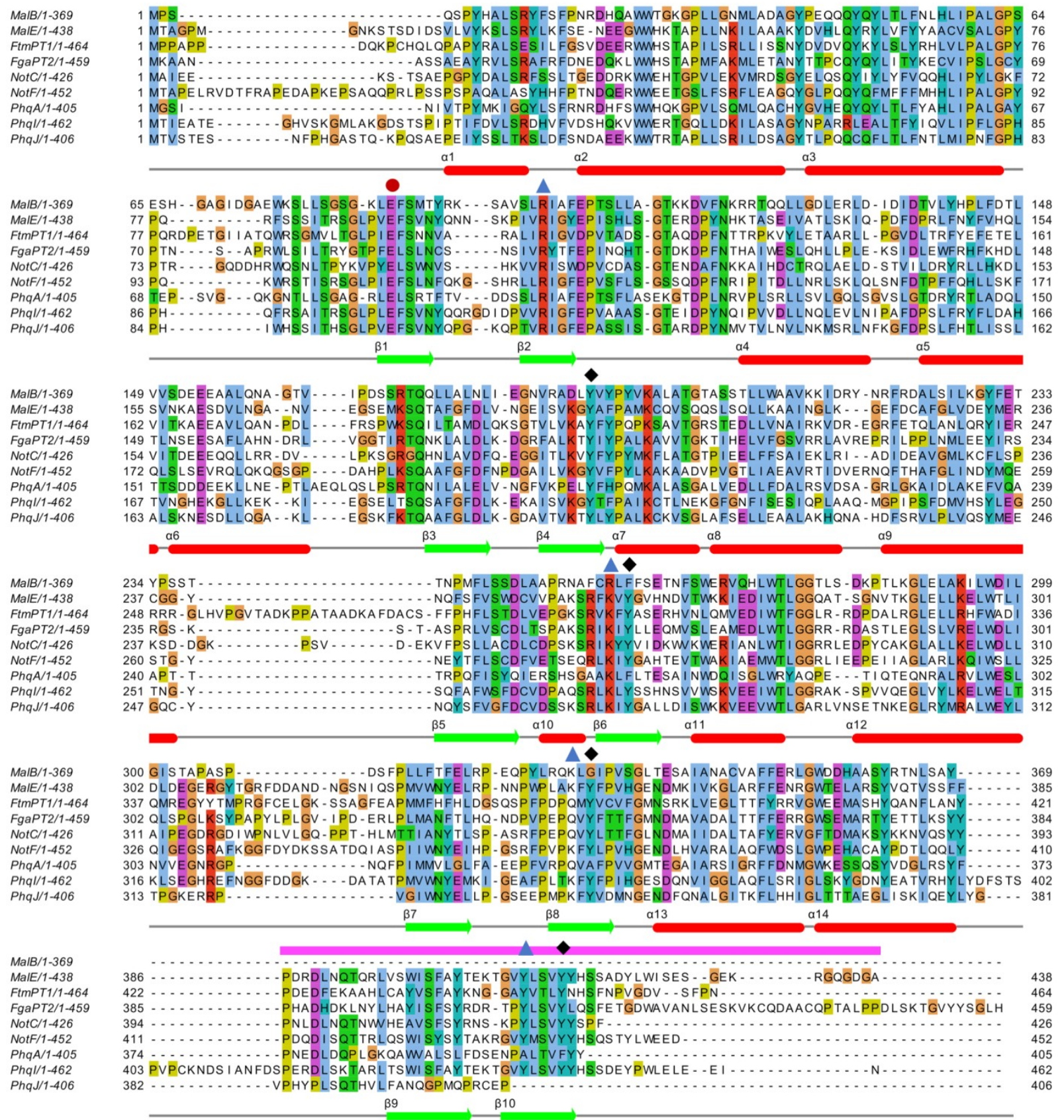

Supplementary Figure 14. Multiple sequence alignment of fungal indole prenyltransferases. The catalytic base Glu is highlighted with a red dot. Residues critical for coordinating the cofactor DMAPP are highlighted with blue triangles. Four Tyr that are expected to shield the active site are highlighted with black squares. MalE contains a full set of conserved residues, while MalB does not. The C-terminal sequence that MalB lacks (magenta) may include the last two $\beta$ strands of the prenyltransferase barrel, possibly contributing to inefficiency of MalB catalysis. 
a

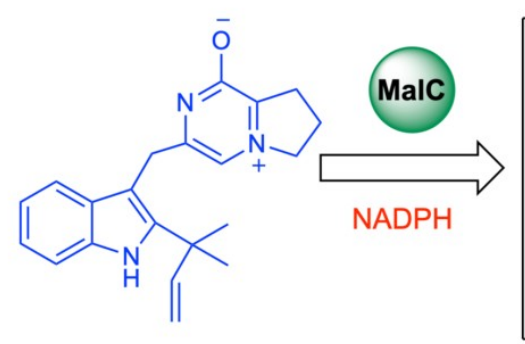

11

b

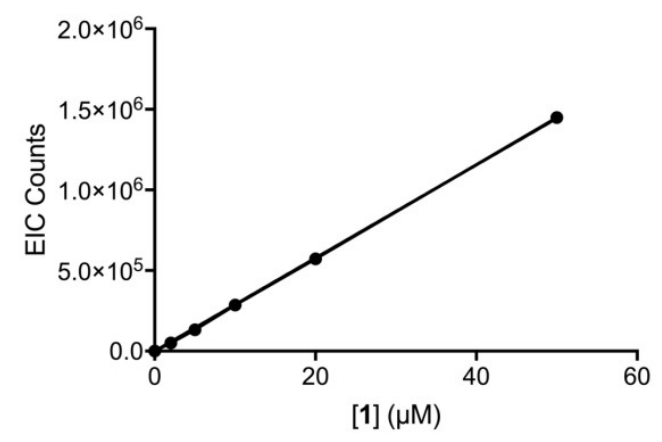

d

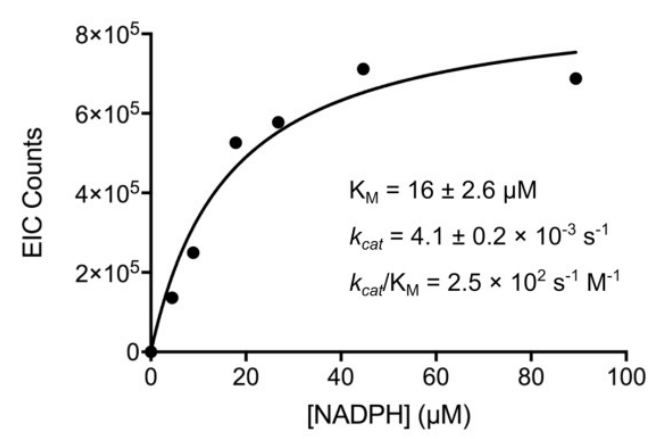

C

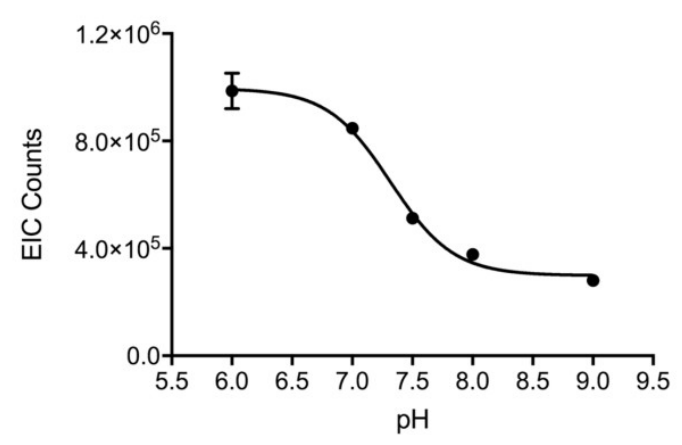

e

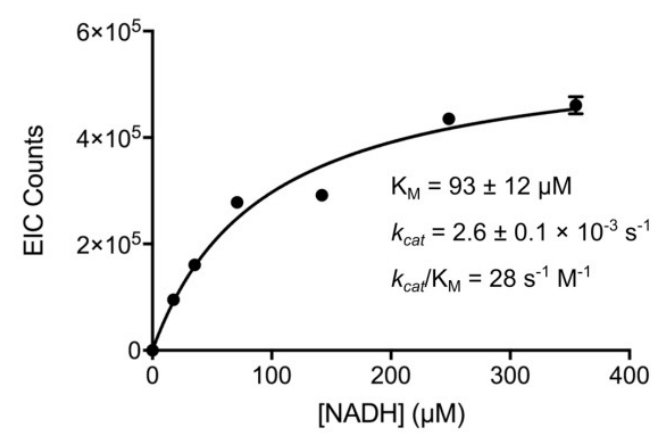

Supplementary Figure 15. a. MalC catalyzes a two-step reaction of $\mathbf{1 1}$ to (+)-1. b. Standard curve of $(+)-1$, presenting linear correlation of EIC counts to $(+)-1$ concentration. c. $\mathrm{pH}$ profile of the MalC-catalyzed reaction, efficiency of which decreases drastically beyond neutral $\mathrm{pH} . \mathrm{d}-\mathrm{e}$. $\mathrm{K}_{\mathrm{M}}$ measurement of NADPH (d) and NADH (e) for MalC catalysis. For all measurements, results were repeated three times (error bars, $\mathrm{SD} ; n=3$ ). 

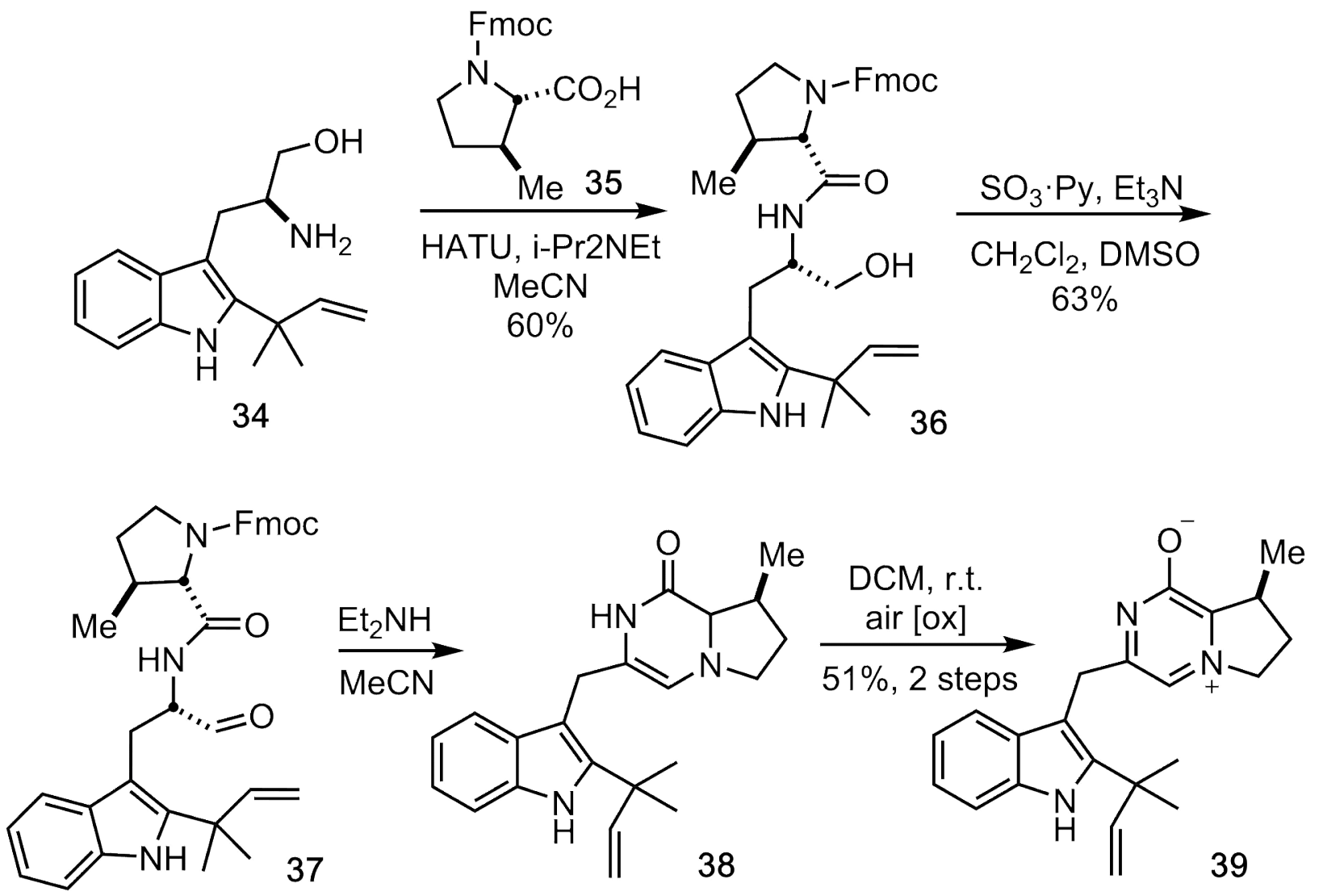

Supplementary Figure 16. Synthetic scheme of $\beta$-methyl prolyl prenyl zwitterion 39, an intermediate in paraherquamide biosynthesis. 
a

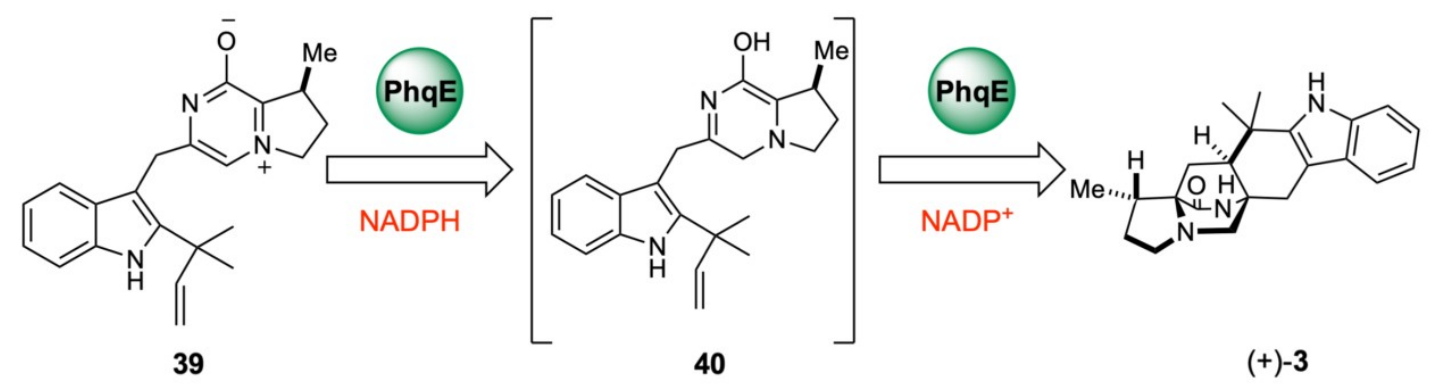

b

In vivo production of $\mathbf{3 9}$

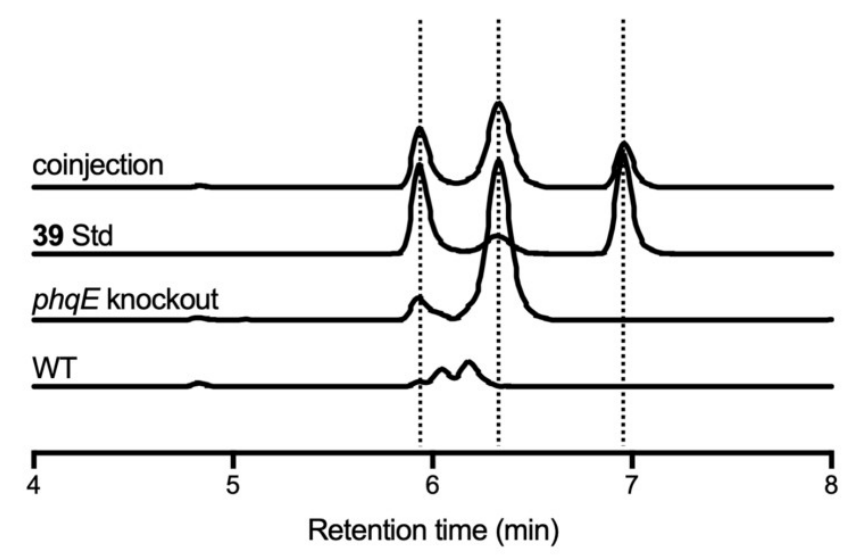

Supplementary Figure 17. Production analysis of $39\left(\mathrm{M}+\mathrm{H}^{+} m / z=348\right)$ by TOF-MS from Penicillium simplicissimum phqE mutant. phqE is the homologous gene of $\mathrm{malC}$ in the paraherquamide biosynthetic pathway. a. PhqE catalyzes a two-step reaction of $\mathbf{3 9}$ to (+)preparaherquamide 3. b. In vivo production of $\mathbf{3 9}$ via $p h q E$ knockout. The EIC traces (from bottom to top) are: 1) Penicillium simplicissimum WT extracts; 2) Penicillium simplicissimum $p h q E$ knockout mutant extracts; 3) 39 authentic standard; 4) Co-injection of $p h q E$ mutant extracts and $\mathbf{3 9}$ standard. 
a

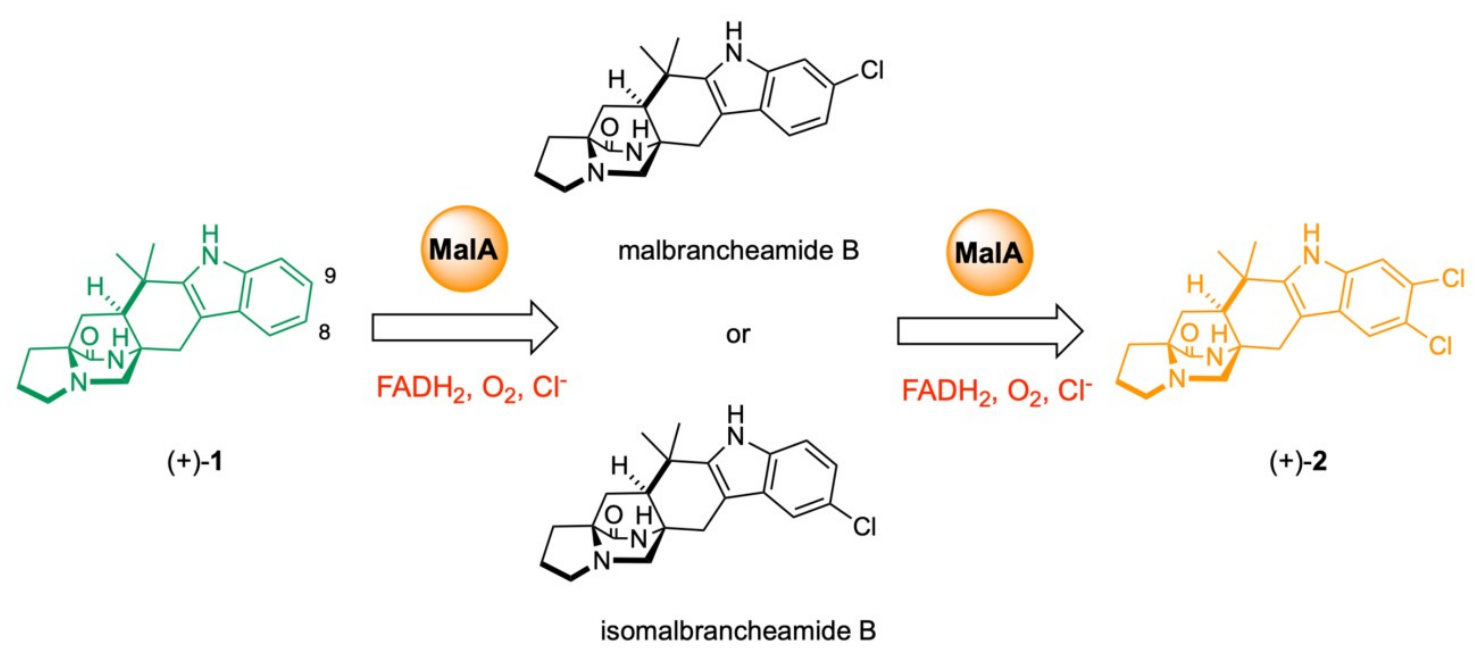

b

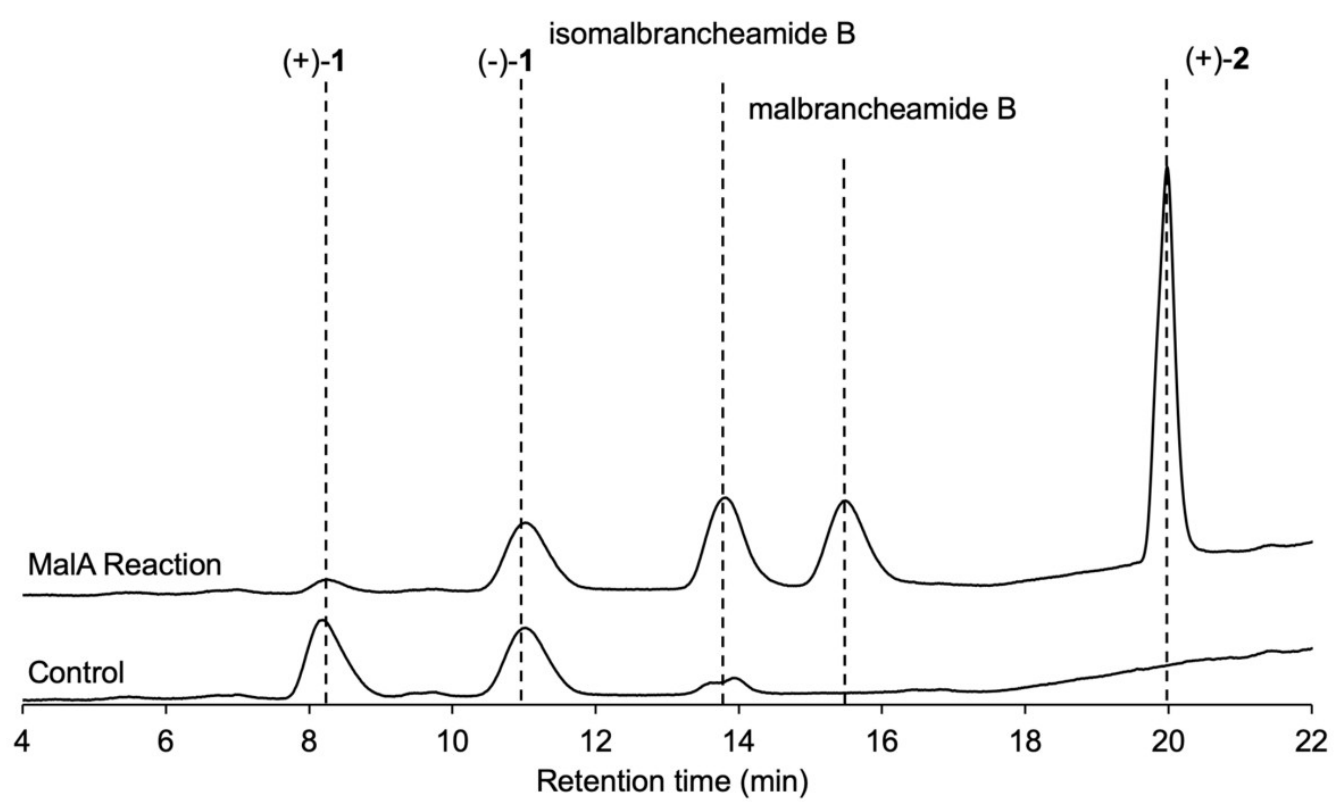

Supplementary Figure 18 a. MalA catalyzes an iterative dihalogenation reaction, converting (+)-1 to (+)-2. b. MalA is stereospecific and does not react on (-)-1. The Y-axis is UV $240 \mathrm{~nm}$ absorbance. 
a

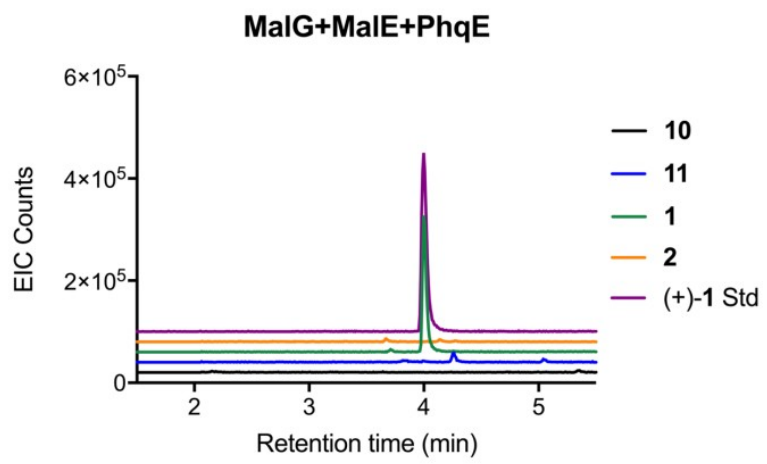

b

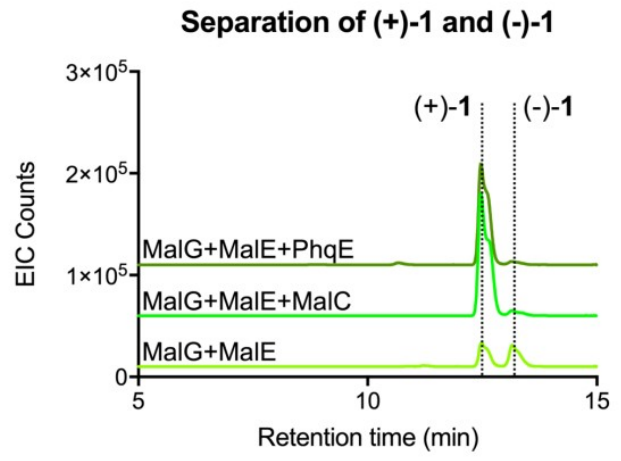

Supplementary Figure 19. PhqE is a bifunctional reductase and Diels-Alderase. a. EIC profile of in vitro malbrancheamide pathway reconstitution assay, with MalC replaced by PhqE. $b$. Chiral separation of (+)-1 and (-)-1, indicating that PhqE is diastereo- and enantioselective. Reconstitution of "MalG+MalE" is shown as a negative control, and "MalG+MalE+MalC" is a positive control. 
a

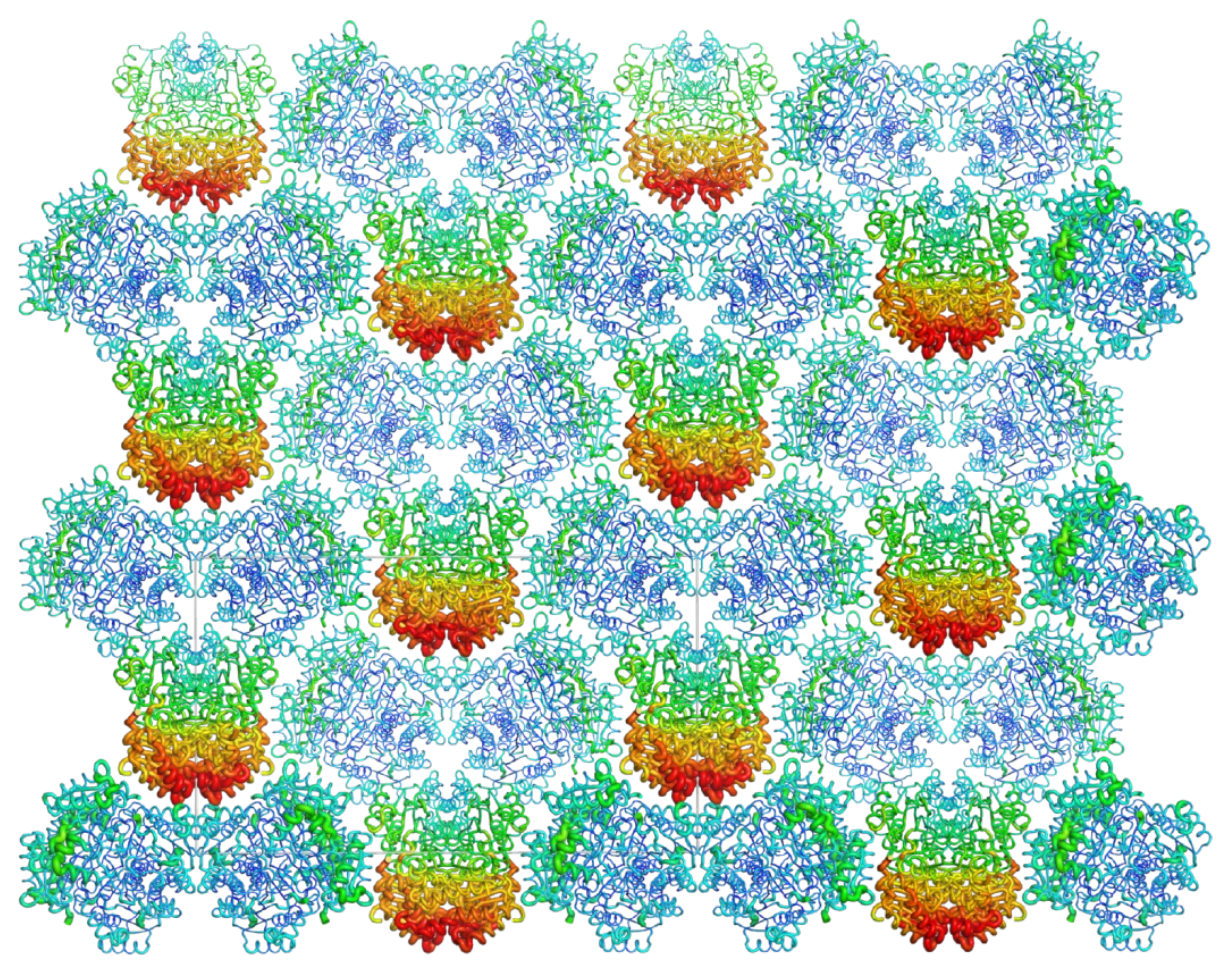

b

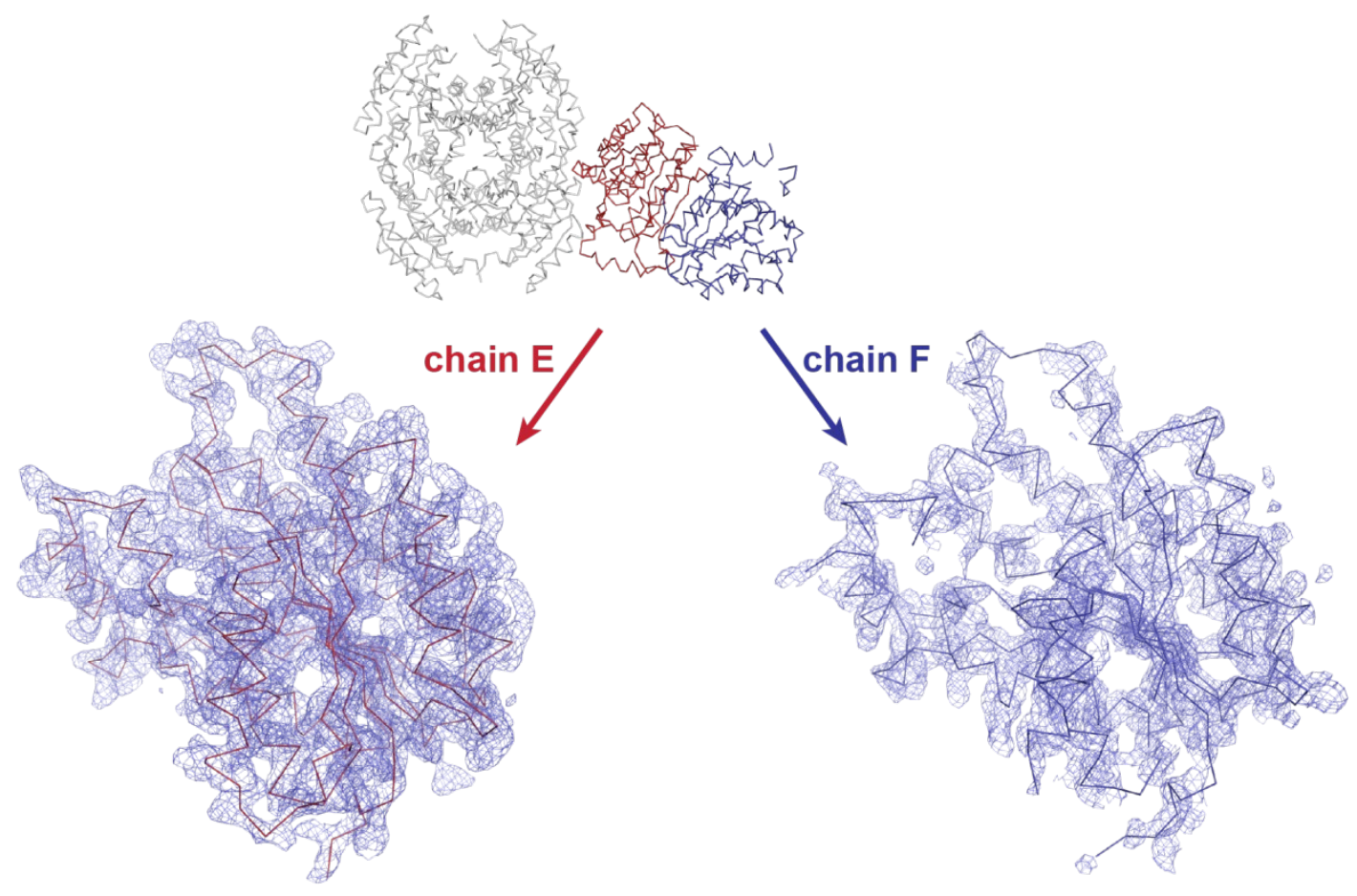

Supplementary Figure 20. PhqE crystal lattice in space group $C 2$. a. Packing diagram colored by $\mathrm{B}$ factor from $20 \AA^{2}$ in blue to $50 \AA^{2}$ in red. The asymmetric unit contains 1.5 tetramers. One tetramer (chains A - D) is well-ordered, while in the half-tetramer (chains $\mathrm{E}$ and $\mathrm{F}$ ) chain $\mathrm{F}$ is 
poorly packed along the crystallographic twofold axis. b. Electron density $(2 \mathrm{Fo}-\mathrm{Fc}$, contoured at $1 \sigma)$ for the E-F half-tetramer showing the poor packing of chain F. 
b
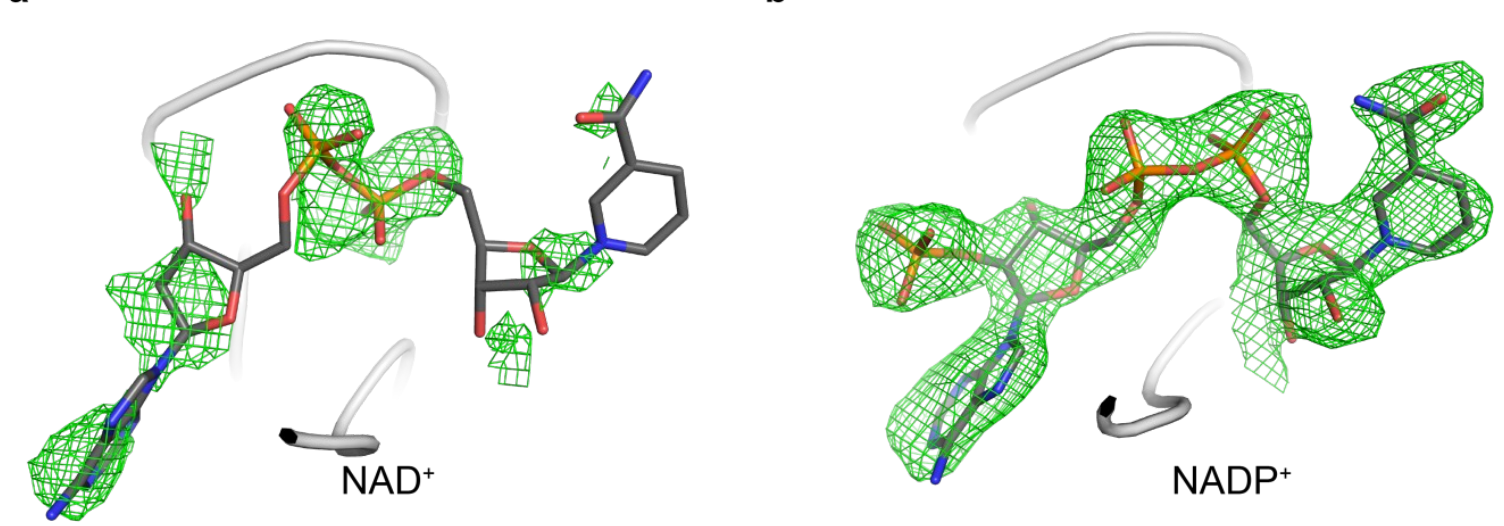

C

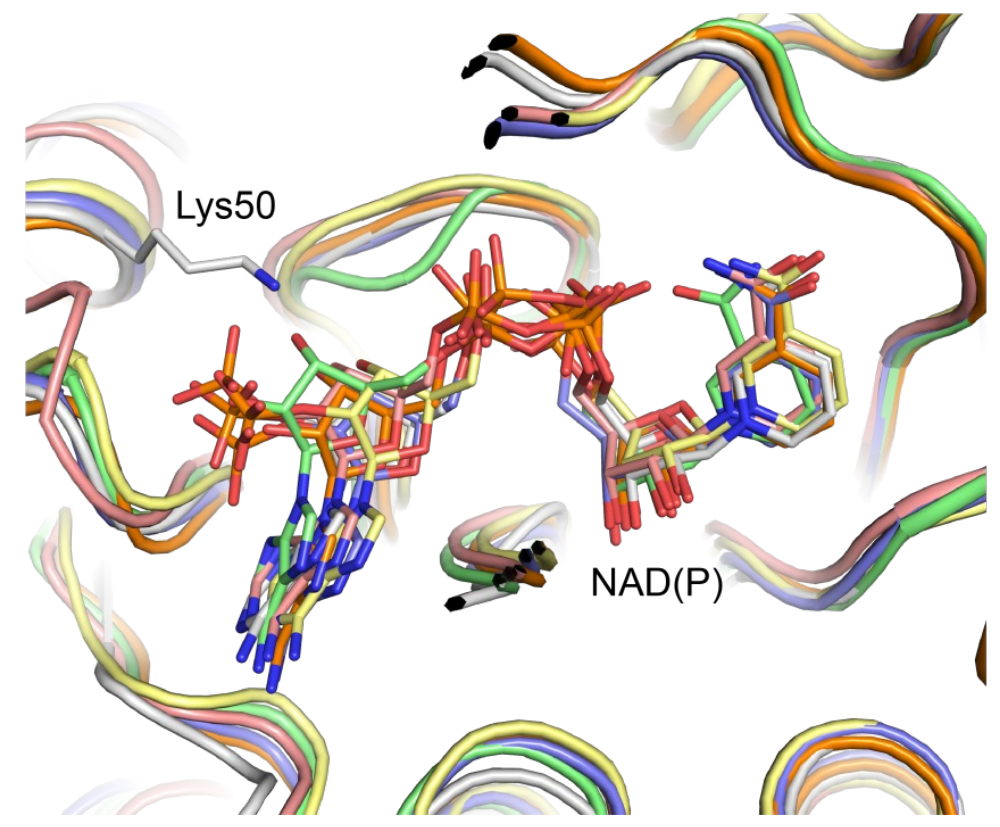

Supplementary Figure 21. Cofactor binding of PhqE. a - b. Poor omit density for $\mathrm{NAD}^{+}$in PhqE (contoured at $2 \sigma$ ) compared to well-ordered $\mathrm{NADP}^{+}$(contoured at $2 \sigma$ ). c. A selection of bacterial SDRs (Burkholderia cenocepacia, PDB 5U2W, pink; Sinorhizobium meliloti, PDB 3TOX, yellow; Ralstonia sp., PDB 4BMS, blue; Bacillus subtilis, PDB 5ITV, orange; Brucella melitensis, PDB 5T5Q, green) superposed on PhqE (gray). The cofactor binding mode and loops surrounding the active site are remarkably similar. 
a

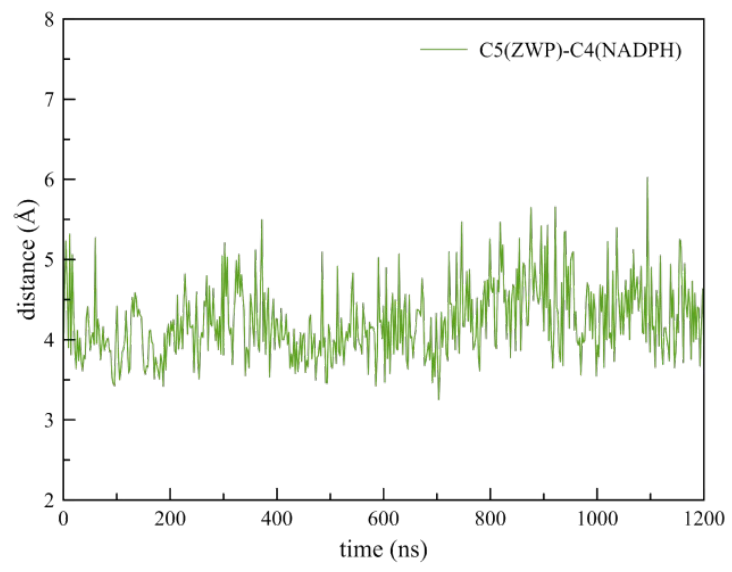

C

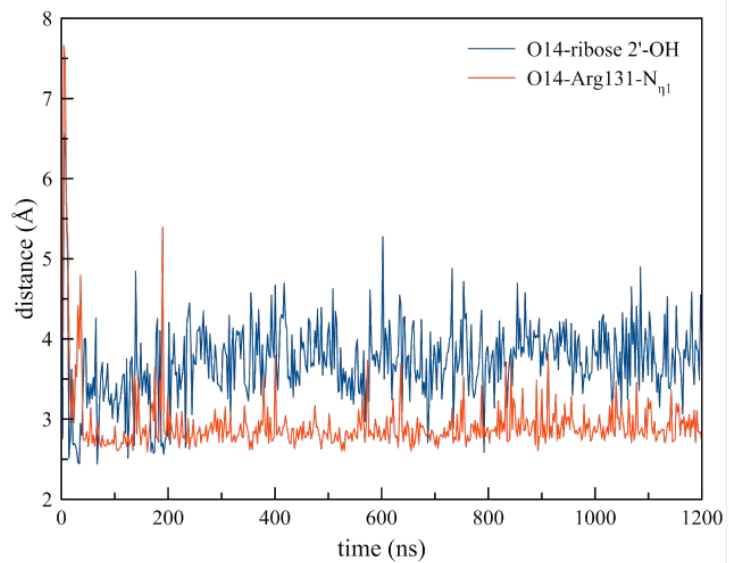

b

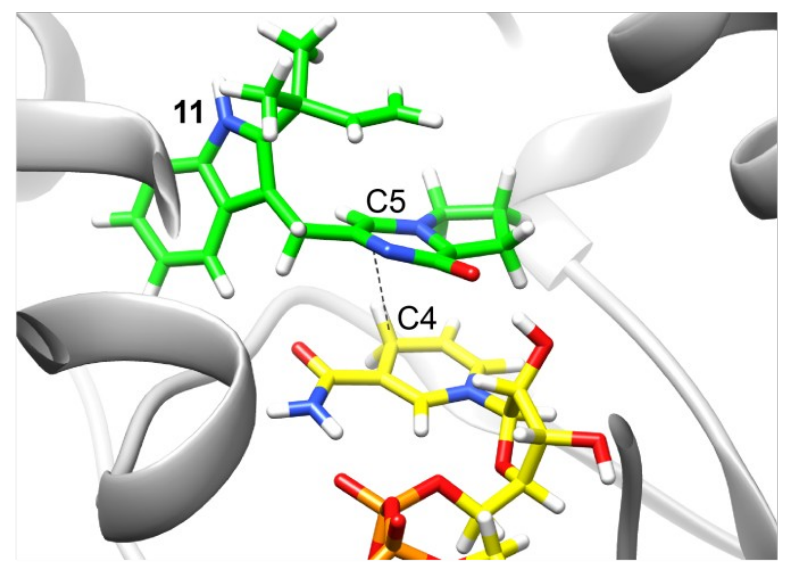

d

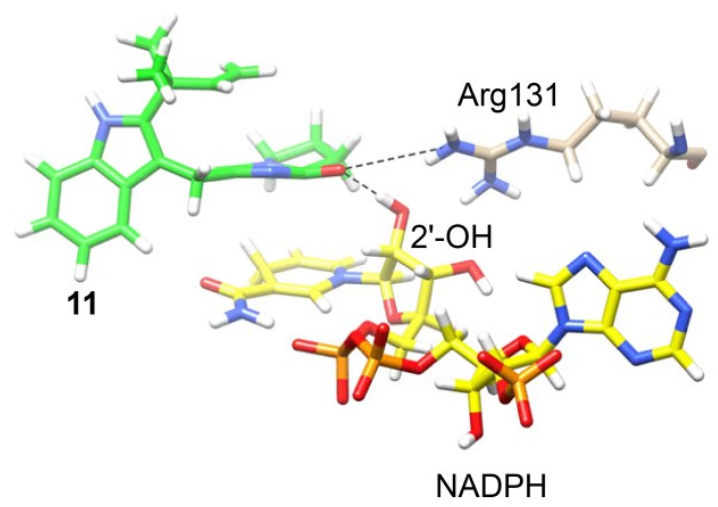

Supplementary Figure 22. a. Plot showing the distance between C5 of $\mathbf{1 1}$ and C4 of NADPH during $1200 \mathrm{~ns}$ simulation; the average distance is $4.4 \AA$. b. Snapshot depicting the atoms. c. Plot showing the distance between the ribose hydroxyl and O14 (blue) and the distance between the $\operatorname{Arg} 131$ and $\mathrm{O} 14$ (red). Arg131 is expected to be protonated at physiological $\mathrm{pH}$, based on the pKa prediction (PROPKA) of 11.10. Arg131 also interacts with Asp109 during the simulation allowing access to bulk solvent. $d$. Snapshot from the beginning of the simulation. 
a

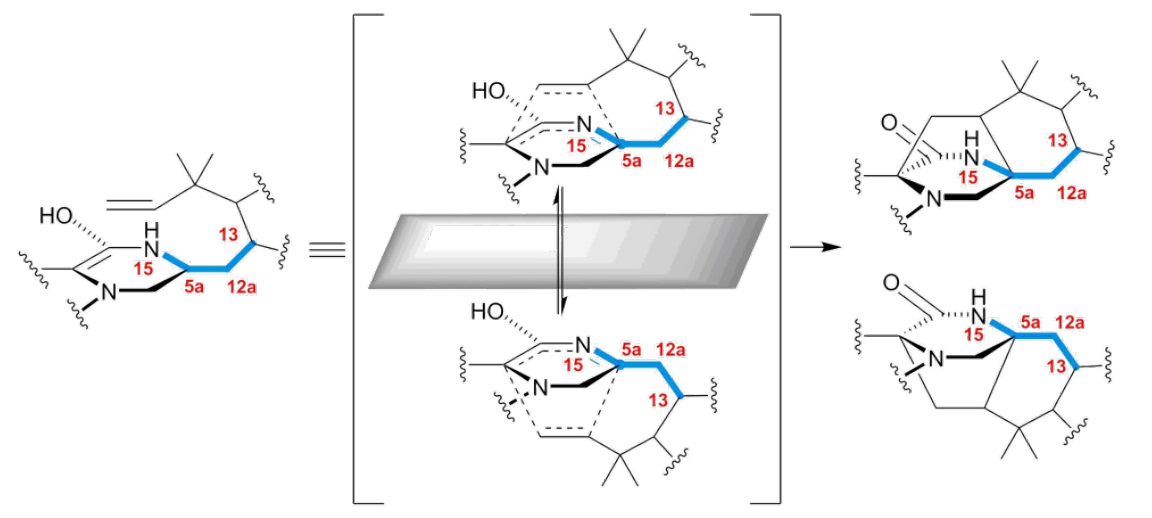

b

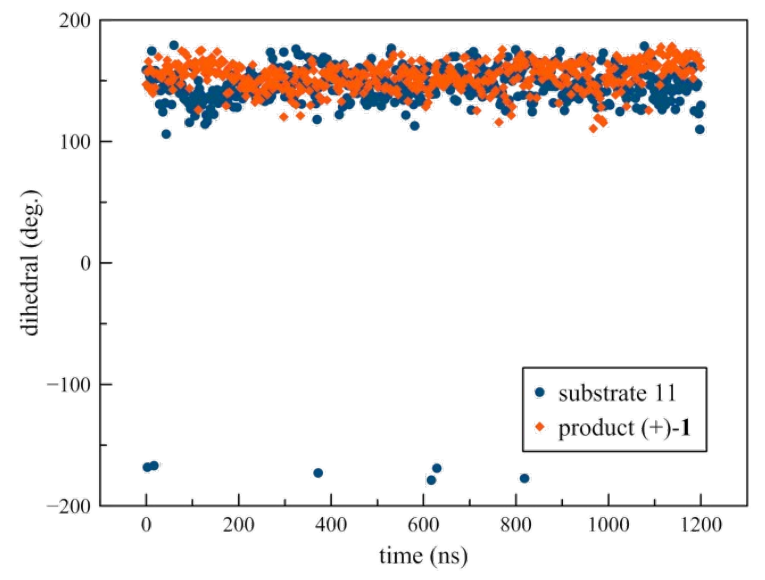

C

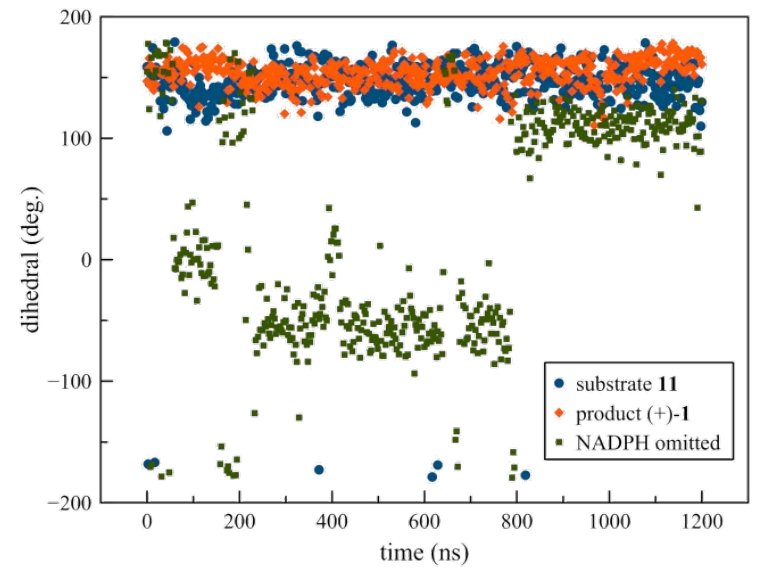

Supplementary Figure 23. a. Dihedral angles monitored to track facial selectivity in [4+2] cycloaddition reaction. b. $1200 \mathrm{~ns}$ MD simulation shows similar dihedrals throughout the simulation despite the fact that the substrate is unconstrained. c. NADP ${ }^{+}$cofactor is required in the simulation to maintain a restrained dihedral angle. 


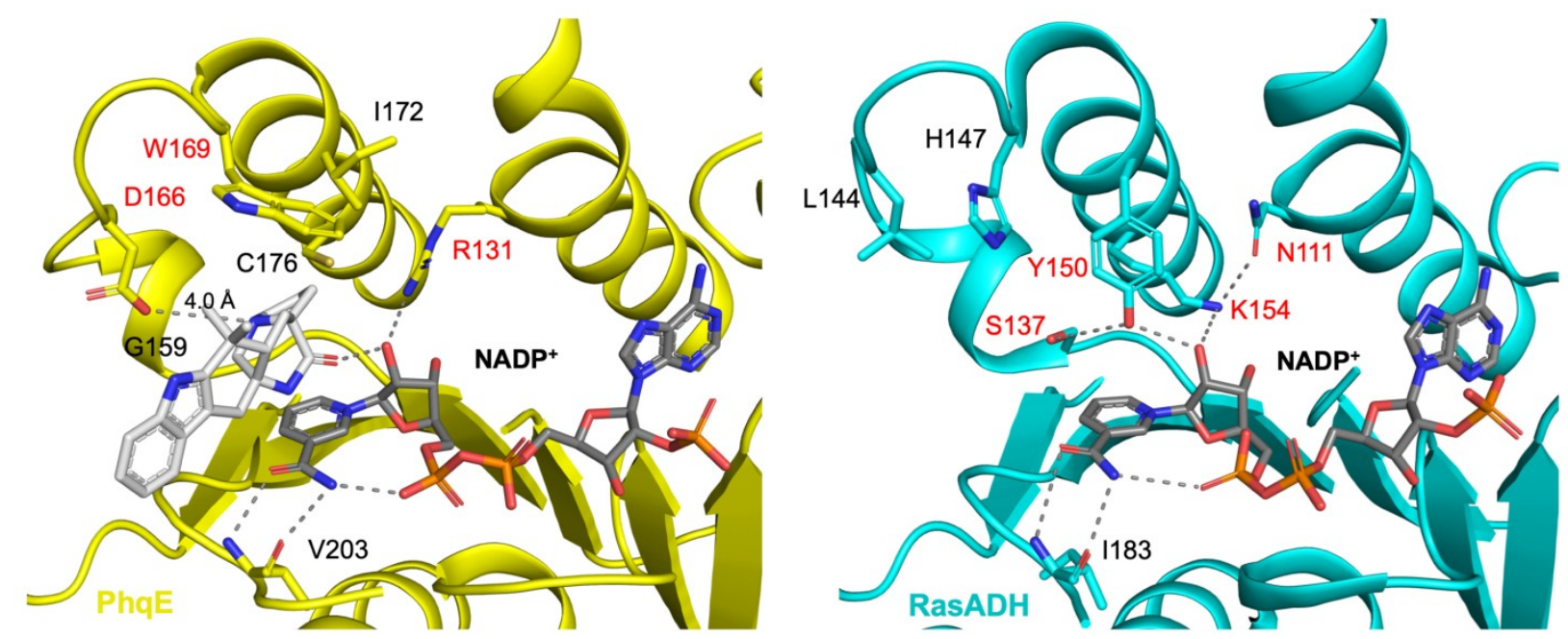

Supplementary Figure 24. Active site comparison of the PhqE product complex at left (yellow, product colored in white) and $\mathrm{RasADH}^{34}$ at right (PDB ID: 4BMS, cyan). For each enzyme, the cofactor is colored in grey, amino acids essential to catalysis are labelled in red, hydrogen bonds are shown as grey dashed lines. Given the striking difference in amino acids in the two active sites, the PhqE and RasADH backbones are remarkably similar. 
MalC[Malbranchea_aurantiaca]//1-264 PhqE[Penicillium fellutanum]/1-265 Unknown1[Aspergillus_turcosus]/1-266 Unknown2[Penicillium_griseofulvum]/1-267 Unknown 3[Penicillium_vulpinum]/1-267 Unknown 4[Penicillium cittrinum]/1-267

Sco1815[Streptomyces]/1-234 FabG[Bacillus]/1-243

FabG[Acholeplasma]/1-240

Dehydrogenase[Brucella]/1-246

Dehydrogenase[Burkholderia]/1-248

MalC[Malbranchea_aurantiaca]/1-264 PhqE[Penicillium fellutanum]/1-265 Unknown 1[Aspergillus_turcosus]/1-266 Unknown2[Penicillium_griseofulvum]/1-267 Unknown 3[Penicillium_vulpinum]/1-267 Unknown4JPenicillium cittrinum]l11-267 RasADH[Ralstonia)/1-249 Sco1815[Streptomyces]/1-234 FabG[Bacillus]/1-243

FabG[Acholeplasma]/1-240 Dehydrogenase[Brucella]/1-246 Dehydrogenase[Burkholderia]/1-248

Ma/C[Malbranchea_aurantiaca]//1-264 PhqE[Penicillium_fellutanum]//1-265 Unknown 1[Aspergillus_turcosus]/1-266 Unknown2[Penicillium_griseofulvum]/1-267 Unknown 3[Penicillium_vulpinum]/1-267 Unknown 4[Penicillium citrinum]1/1-267 RasADH[Ralstonia]/1-249

Sco1815[Streptomyces]/1-234 FabG[Bacillus]/1-243

FabG[Acholeplasma]/1-240 Dehydrogenase[Brucella]/1-246 Dehydrogenase[Burkholderia]/1-248

MalC[Malbranchea_aurantiaca]/1-264 PhqE[Penicillium_fellutanum]//1-265 Unknown1[Aspergillus_turcosus]/1-266 Unknown2[Penicillium griseofulvum]/1-267 Unknown 3[Penicillium vulpinum]/1-267 Unknown 4[Penicillium citrinum]l/1-267 RasADH[Ralstonia]/1-249 Sco1815[Streptomyces]/1-234 FabG[Bacillus]/1-243

FabG[Acholeplasma]/1-240 Dehydrogenase[Brucella]/1-246 Dehydrogenase[Burkholderia]/1-248

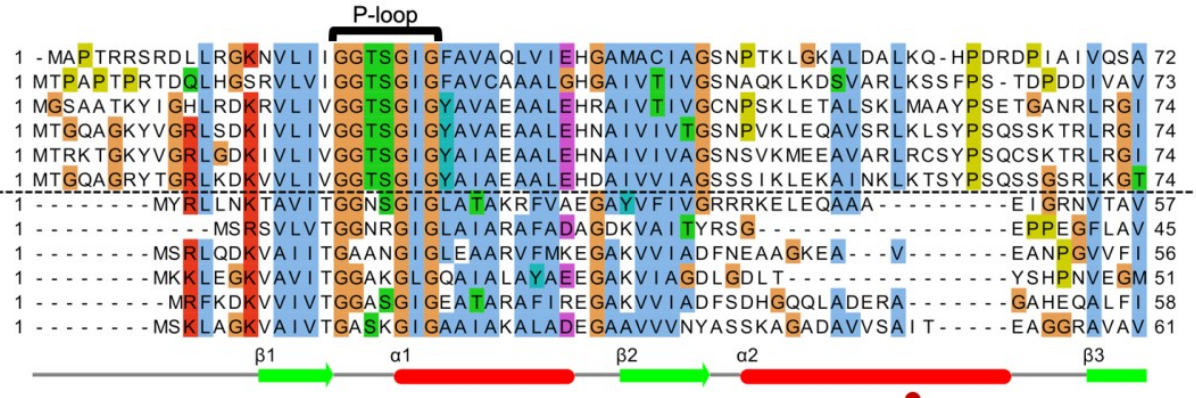

73 TCDLFDVPNLEQNLDNLLKLAAGDSKI HHIVFTAADMVQPP - PLASVTIEQI QRVGTIRFTAPMLVAKLLPKYM 145 74 RCDLSNSDTVEQD I EKALQLAAGNSK I NHI V I TAADMTAPP - PLEDL TVDSVQRPGI I RLVAPLMVAKHLPKYM 146 75 PCDLGDADQLE TNVQKVLGFAAAGSK I NHI V I TAADMTQPP - S I AKV TAEN I QR TGT I RYLAPL I FAKHLPQFM 147 75 TI DLADAENMEENVQS LRFSANGAK I NHV I I TAADMMPPPPPLAE I TVHSVERSGR I RY TAPL I FAKY LPQFM 148 75 TI DLADGENLEANVQSVLRFAANGAK I NHV I I TAADMMPPPPP LAG I TVQSLERSGR I RY TAPLI FAKYLPQFM 148 75 TIDLADIENLEKNVQSVLQLAAEGVK INHFVVTAADMMPPPPPLAEMTVHSLERTSCIRYLAPLI FAKHLPKYM 148 58 KADVTKLEDLDRLYA IVREQ- - RGS I D - VLFANS GA I EQK - TLEEI TPEHYDRTFDVNVRGL I FTVQKALPLL 126 46 KCD I TDTEQVEQAYKE I EET - . - HGPVE - VL I ANAGV TKDQ - LLMRMSEEDFTSVVE TNLTGTFRVVKRANRAM 114 57 RVDVSDRESVHRLVENVAER - . - FGK I D - I L I NNAGI TRDS - MLSKMTVDQFQQV I NVNLTGVFHCTQAVLPYM 125 57 RVDVSDRESVHRLVENVAER - - - FGK I D - I L I NNAG I TRDS - MLSKMTVDQFQQV I NVNL TGV FHC TQAVLPYM 125
52 YLNVTDVTGVEK FYQSV I DK - - - YGK I D - I LVNNAG I TKDA - MTRKMTEAQWDAV I DVNLKGVFNL TRLVGPQM 120 52 Y LNV TDV TGVEK FY SSV I DK - - - YGK I D - I LVNNAG I TKDA - MTRKMTEAQWDAV I DVNLKGV FNL TRLVGPQM 120
59 KTDVADTRAVQAL I ARTVEN - - YGRLD - I MFANAG I AADA - P I DE LDEAAWQK T I D I NL TGVYLCDKYA I DQM 127 62 GGDVSKAADAQR I VDTA IET - . - YGRLD - VLVNNSGVYE FA - PIEAI TEEHYRRQFDTNVFGVLLTTQAAVKHL 130 a3 a4

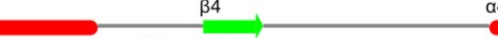

146 ELCPENSYTLTSGSHAKQPDPGWSLVTGYCGGVEGLMRGLAVDMMP - . - - LRVNVVSPGAVLTPVLRDI LG - . - 212 147 NKCPQSS L TL TSGAHCLRPDPGWTV I SGYCGAVEAMSRGLA I DLKP - . - LRVNVVAPGAVL TEAVKDI LG - . - 213 148 ELES GNSMTL TS GAHAHKPDP GWTV I SAYCGAVESMAKGLA I DLKP - - - LRVNAVSPGA I MTDVVKDI LG - - - 214 149 DRASENSL TL TSGAHGHKPDPGWSA I I GYLGAVETMMKGLAVDLKP - . - - LRVNVVSPGAVMTEVVREMLG - - 215 149 DRVSENS I TL TSGAHGHKPDPGWSA I TGY I GAVE TMMKGLAVDLKP - - - - LRVNVVSPGA I MTEVVRQMLG - - - 215 149 DQVPENSVTLTS GAHGHKPDPGWSAMNGYLGAVETMMKGLAVDLKP ... - LRVNVVSPGAV I TE IVRD I LG . . 215 127 RD- -GGSVILTSSVAGVLGLQAHDTYSAAKAAVRSLARTWT TELKG--RS IRVNAVSPGAIDTPIIENQVSTQE 196 115 LRAKKGRVVLIISSVVGLLGSAGQANYAASKAGLVGFARSLARELGS - - RNI TFNVVAPGFVDTDMTK - . - . - V 180 126 AEQGKGK I I NTSSVTGTYGNVGQTNYAAAKA GV I GMTKTWAKELAR - - KGI NVNAVAPGFTETAMVA - . - . - E 191 121 QTNGYGS I I N I S SVVGVFGN I GQANYAA TKAGV I GL TMTWAKEFALKGANVRVNA I APGY I MTD I LK - . - . - T 188 128 RSQGGGV I VNCGS I HSHVGKSGVTAYAAAKGGVKLLTQTLA I DYGP - - QN I RVNAVCPGYI DTPLLK - . - . - N 193 $131 \mathrm{GE}$ - - GAS I I N I SSVVTSI TPPASAVYSGTKGAVDA I TGVLALELGP - - RKI RVNA INPGMIVTEGTHSA - - - GI 197 a5 $\beta 6$ a6

213 DSLE I ALDAARKKSTTGR I ARPEDVAEAYLYI MKD - - QN I TGTVLETSAGMLL - R 264 214 DAYDAAVEMAEAKS TVGQTGSPESVAQAY I YLMKD - - HYASGSVVSTNGGMLL - V 2265 215 DAYDFA I RMAKEKSLVASAGAPEQVAQAYLYLMKD - - QYVSGS I I ESNGGML L - V 26 216 PHYDMA I QMAKEKSLVGGAGTPESVAQAYLYLMKD - - QFV TGTVLESNGGLML - V 267 216 PHYDMA I QMAKEKSLVGSAGTPESVAQAYLYLMKD - - PFVTGTVLESNGGLLL - A 267 216_EHYDMA IQMAKEKS SLVGGAGTPESVAQA YLYLMKD - - SFVITIVLETNGGLYL - A 197 EADELRAKF - AAATPLGRVGRPELLAAAVLFLASDDS Y VAGIELFVDGGLTQ-V 181 LTDEQRAN I - VSQVPLGRYARPEE I AA TVRFLASDDASY I TGAV I PVDGGLGMGH 23 192 VPEKV I EKM-KAQVPMGRLGKPEDIANAYLFLASHESDYVNGHVLHVDGGI M - - M 243 189 VPQD LLDK F - AA L TMLNRL GQPEE I AKVAL FLASDDASYV TGQT I NVNGGMR - - L 240 194 IPDDKKQAL - VALHPMGRLGRAEEVANAVLFLASDEASFVNGASLLVDGGYTA-Q 198 I GSDLEAQV - LGQTPLGRLGEPND I ASVAVFLASDDARWMTGEHLVVSGGLN - - a7 a8 $\beta 7$

Supplementary Figure 25. Multiple sequence alignment of MalC and PhqE homologs. The Ploop critical for cofactor binding is highlighted with a black cap, the "PDPGW" motif is highlighted with a purple cap. SDR amino acids essential for catalysis (Asn-Ser-Tyr-Lys) are shown with red dots, all of which are different in the MalC/PhqE-type Diels-Alderases. In order to validate the reliability of the unknown sequences, we identified and annotated two fungal genomes (Aspergillus turcosus, GenBank accession number NIDN01000061; Penicillium griseofulvum, GenBank accession number LHQR01000065; Table S3 ${ }^{42}$ ), confirming that both contain clustered homologs of $\mathrm{malG}$, malE and $\mathrm{malC}$, and revealing more potential pathways that produce the bicyclo[2.2.2]diazaoctane nucleus. Sequences below the dashed line are conventional SDRs of known structure. 


\section{Supplementary Table 1. Oligonucleotides used in this study}

\begin{tabular}{|c|c|c|}
\hline Gene & Primer Direction & Primer Sequence \\
\hline \multirow{2}{*}{ malG $A_{I}-T_{I}(198-838)$} & Forward & 5'-TACTTCCAATCCAATGCCTTGATGTGTGAGTCCGATATCGAA-3' \\
\hline & Reverse & 5'-TTATCCACTTCCAATGCTAAGCAGAGATCATGGTTCCAGCA-3' \\
\hline \multirow{2}{*}{$\operatorname{malG} C(846-1277)$} & Forward & 5'-TACTTCCAATCCAATGCCTCAAAGACATCATTCGCCATTAACAAAT-3' \\
\hline & Reverse & 5'-TTATCCACTTCCAATGCTATTGTCGGTATGGGTCAACCTC-3' \\
\hline \multirow{2}{*}{$\operatorname{malG} T_{2}(1841-1925)$} & Forward & 5'-TACTTCCAATCCAATGCCACACTTCAACCTCACGAAAGCAC-3' \\
\hline & Reverse & 5'-TTATCCACTTCCAATGCTAAACCCCTTCAATGAGCCTGG-3' \\
\hline \multirow{2}{*}{$\operatorname{malG} R(1932-2345)$} & Forward & 5'-TACTTCCAATCCAATGCCTCACAATTCGATCTCTATGCCAAGTA-3' \\
\hline & Reverse & 5'-TTATCCACTTCCAATGCTATCACAGGACGCGTCTAAAAATACG-3' \\
\hline \multirow{2}{*}{ malE } & Forward & 5'-TACTTCCAATCCAATGCCATGACAGCAGGTCCGATGG-3' \\
\hline & Reverse & 5'-TTATCCACTTCCAATGCTATCAAGCACCATCTCCTTGACC-3' \\
\hline \multirow{2}{*}{ malB } & Forward & 5'-TACTTCCAATCCAATGCCATGCCTTCACAAAGCCCATATCAT-3' \\
\hline & Reverse & 5'-TTATCCACTTCCAATGCTACTAGTAAGCTGACAAGTTGGTTCG-3' \\
\hline \multirow{2}{*}{ malC } & Forward & 5’-TACTTCCAATCCAATGCCATGGCACCTACCAGGAGATC-3' \\
\hline & Reverse & 5'-TTATCCACTTCCAATGCTATCAGCGCAAAAGCATCCCC-3' \\
\hline \multirow{2}{*}{$p h q B R(2006-2449)$} & Forward & 5'-TACTTCCAATCCAATGCCTGGTGGGAGAGGGTGCAA-3' \\
\hline & Reverse & 5'-TTATCCACTTCCAATGCTATTAAGAGTTGATAAGACCATTCCC-3' \\
\hline \multirow{2}{*}{$p h q B R(2006-2429)$} & Forward & 5'-TACTTCCAATCCAATGCCTGGTGGGAGAGGGTGCAA-3' \\
\hline & Reverse & 5'-TTATCCACTTCCAATGCTAGGCAGCCAGGTCCTCAAG-3' \\
\hline \multirow{2}{*}{$p h q E$} & Forward & 5'-GATCCAGCTAGCATGACACCCGCTCCGACACCAC-3' \\
\hline & Reverse & 5'-ATCAGACTCGAGTTAGACAAGAAGCATGCCACCGTTTG-3' \\
\hline \multirow{2}{*}{ malG $R Y 2132 F$} & Forward & 5'-CGTATTTGGTCTGACTGAAGCCATCATCGGAGGTT-3' \\
\hline & Reverse & 5'-AACCTCCGATGATGGCTTCAGTCAGACCAAATACG-3' \\
\hline \multirow{2}{*}{ malC D108A } & Forward & 5'-GGCTGCACCATGGCTGCGGCGGTGA-3' \\
\hline & Reverse & 5'-TCACCGCCGCAGCCATGGTGCAGCC-3' \\
\hline \multirow{2}{*}{ malC D108N } & Forward & 5'-GCTGCACCATGTTTGCGGCGGTGAAGAC-3' \\
\hline & Reverse & 5'-GTCTTCACCGCCGCAAACATGGTGCAGC-3' \\
\hline \multirow{2}{*}{ malC R130A } & Forward & 5'-CATCGGTGCTGTAAATGCAATCGTGCCAACGCGC-3' \\
\hline & Reverse & 5'-GCGCGTTGGCACGATTGCATTTACAGCACCGATG-3' \\
\hline \multirow{2}{*}{ malC R130K } & Forward & 5'-AGCATCGGTGCTGTAAATTTAATCGTGCCAACGCGCTG-3' \\
\hline & Reverse & 5'-CAGCGCGTTGGCACGATTAAATTTACAGCACCGATGCT-3' \\
\hline \multirow{2}{*}{ malC $R 130 Q$} & Forward & 5'-CATCGGTGCTGTAAATTGAATCGTGCCAACGCG-3' \\
\hline & Reverse & 5'-CGCGTTGGCACGATTCAATTTACAGCACCGATG-3' \\
\hline \multirow{2}{*}{ malC H160A } & Forward & 5'-ATCGGGCTGTTTTGCAGCCGATCCGCTGGTCAAG-3' \\
\hline & Reverse & 5'-CTTGACCAGCGGATCGGCTGCAAAACAGCCCGAT-3' \\
\hline \multirow{2}{*}{ malC D165A } & Forward & 5'-GACTCCATCCCGGAGCGGGCTGTTTTGCA-3' \\
\hline & Reverse & 5'-TGCAAAACAGCCCGCTCCGGGATGGAGTC-3' \\
\hline \multirow{2}{*}{ malC D165N } & Forward & 5'-GACTCCATCCCGGATTGGGCTGTTTTGCATG-3' \\
\hline & Reverse & 5'-CATGCAAAACAGCCCAATCCGGGATGGAGTC-3' \\
\hline \multirow{2}{*}{ malC $W 168 F$} & Forward & 5'-CCCGTAACAAGACTGAATCCCGGATCGGGCTG-3' \\
\hline & Reverse & 5'-CAGCCCGATCCGGGATTCAGTCTTGTTACGGG-3' \\
\hline \multirow{2}{*}{ malC $W 168 L$} & Forward & 5'-CCGTAACAAGACTCAATCCCGGATCGGGCT-3' \\
\hline & Reverse & 5'-AGCCCGATCCGGGATTGAGTCTTGTTACGG-3' \\
\hline
\end{tabular}




\section{Supplementary Table 2. Crystallographic table}

\begin{tabular}{|c|c|c|c|c|}
\hline & PhqB R $\cdot$ NADPH & $\mathrm{MalC}$ & $\begin{array}{c}\text { PhqE D166N } \cdot 11 \\
\cdot \text { NADP }^{+} \\
\end{array}$ & $\begin{array}{c}\text { PhqE } \cdot 1 \cdot 1 \cdot \\
\text { NADP }^{+} \\
\end{array}$ \\
\hline \multicolumn{5}{|l|}{ Data collection } \\
\hline Space group & $I 222$ & $P 4_{2}$ & & \\
\hline \multicolumn{5}{|l|}{ Unit cell parameters } \\
\hline$a, b, c(\AA)$ & $81.6,91.6,124.6$ & $79.4,79.4,133.6$ & & \\
\hline$\alpha, \beta, \gamma\left(^{\circ}\right)$ & $90,90,90$ & $90,90,90$ & & \\
\hline Wavelength $(\AA)$ & 1.033 & 1.033 & & \\
\hline $\mathrm{d}_{\min }(\AA)$ & $\begin{array}{c}2.60 \\
(2.69-2.60)^{*}\end{array}$ & $\begin{array}{c}1.60 \\
(1.66-1.60)\end{array}$ & & \\
\hline Completeness (\%) & $99.8(99.1)$ & $99.2(92.1)$ & & \\
\hline Multiplicity & $13.3(12.7)$ & $6.6(5.0)$ & & \\
\hline Mean $I / \sigma$ & $20.5(1.1)$ & $15.4(1.4)$ & & \\
\hline $\mathrm{R}_{\text {meas }}$ & $0.074(2.47)$ & $0.073(1.08)$ & & \\
\hline $\mathrm{CC}_{1 / 2}$ & $1(0.64)$ & $1(0.45)$ & & \\
\hline \multicolumn{5}{|l|}{ Refinements } \\
\hline Data range $(\AA)$ & $45.82-2.60$ & $42.97-1.60$ & & \\
\hline No. Reflections & 14738 & 107863 & & \\
\hline $\mathrm{R}_{\text {work }}$ & 0.26 & 0.18 & & \\
\hline $\mathrm{R}_{\text {free }}$ & 0.30 & 0.21 & & \\
\hline No. of Atoms & 2755 & 8399 & & \\
\hline Protein & 2707 & 7735 & & \\
\hline Ligands & 48 & -- & & \\
\hline Water & -- & 664 & & \\
\hline Avg $B$-values $\left(\AA^{2}\right)$ & 123.0 & 27.8 & & \\
\hline Protein & 121.7 & 27.6 & & \\
\hline Ligands & 192.0 & -- & & \\
\hline Water & -- & 29.9 & & \\
\hline $\begin{array}{l}\text { Ramachandran plot: } \\
\text { favored/allowed/outlier } \\
(\%)\end{array}$ & $95.6 / 4.4 / 0$ & $98.5 / 1.5 / 0$ & & \\
\hline RMSD bonds $(\AA)$ & 0.016 & 0.008 & & \\
\hline RMSD angles $\left({ }^{\circ}\right)$ & 1.61 & 1.06 & & \\
\hline
\end{tabular}

*Values in parentheses are for the highest-resolution shell. 


\section{Supplementary Table 3. Gene cluster annotation of $\mathrm{mal} / \mathrm{phq}$ homologous pathways}

\begin{tabular}{|c|c|c|c|c|}
\hline \multicolumn{5}{|c|}{ Aspergillus turcosus (GenBank accession number NIDN01000061) } \\
\hline ORF & $\begin{array}{l}\text { Size } \\
\text { (aa) }\end{array}$ & Putative Function & Relative identity/similarity (\%) & Accession No. \\
\hline 1 & 266 & short-chain dehydrogenase & [Penicillium fellutanum $](58 / 75) ; p h q E$ & AGA37272.1 \\
\hline 2 & 773 & $\begin{array}{l}\text { P-loop containing nucleoside } \\
\text { triphosphate hydrolase protein }\end{array}$ & $\begin{array}{c}\text { [Aspergillus steynii IBT 23096], multi-drug } \\
\text { resistance }\end{array}$ & PLB53566.1 \\
\hline 3 & 599 & L-amino-acid oxidase & [Madurella mycetomatis] $(55 / 70)$ & KXX80598.1 \\
\hline 4 & 1080 & NRPS & [Aspergillus oryzae $](39 / 57)$ & OOO14897.1 \\
\hline 5 & 455 & cytochrome P450 & [Penicillium griseofulvum] $(44 / 60)$ & KXG49078.1 \\
\hline 6 & 445 & FAD monooxygenase & [Penicillium oxalicum] (37/59); phqK & AOC84388.1 \\
\hline 7 & 330 & cytochrome P450 & [Penicillium griseofulvum] $(61 / 76)$ & KXG49078.1 \\
\hline 8 & 411 & prenyltransferase & [Malbranchea aurantiaca] $(56 / 74) ;$ malE & AGA37265.1 \\
\hline 9 & 308 & negative regulator & [Penicillium fellutanum] $(65 / 76) ; p h q G$ & AGA37274.1 \\
\hline 10 & 364 & prenyltransferase & [Malbranchea aurantiaca] (41/59); malE & AGA37265.1 \\
\hline 11 & 1048 & hypothetical protein CFD26_02683 & [Aspergillus turcosus] $(89 / 90)$ & OXN18465.1 \\
\hline 12 & 323 & 2OG-Fe(II)-oxygenase & [Penicillium fellutanum] (41/57); phqC & $\begin{array}{l}\text { AGA37270.1 } \\
\text { AFT91382.1 } \\
\end{array}$ \\
\hline 13 & 2324 & NRPS & {$[$ Malbranchea aurantiaca $]$ (41/58); malG } & AGA37267.1 \\
\hline 14 & 502 & cytochrome P450 & [Aspergillus ruber CBS 135680] (48/64) & EYE91288.1 \\
\hline 15 & 420 & P450 monooxygenase & [Penicillium fellutanum] (38/57); phqM & AGA37280.1 \\
\hline 16 & 295 & methyltransferase & [Aspergillus ochraceoroseus IBT 24754] (33/50) & PLB24695.1 \\
\hline 17 & 2553 & $\begin{array}{c}\text { Type I Iterative Polyketide synthase } \\
\text { (PKS) }\end{array}$ & [Pseudogymnoascus sp. 23342-1-I1] (41/59) & OBT66706.1 \\
\hline 18 & 363 & cytochrome P450 & [Aspergillus oryzae $](42 / 62)$ & OOO07737.1 \\
\hline 19 & 327 & Phytanoyl-CoA dioxygenase & [Penicillium expansum] $(36 / 54)$ & XP_016600816.1 \\
\hline 20 & 350 & $\begin{array}{l}\text { putative Proline utilization protein } \\
\text { PrnX } \\
\end{array}$ & [Aspergillus calidoustus] (57/73) & CEL10788.1 \\
\hline 21 & 492 & transcriptional regulator & [Quercus suber] & XP_023878682.1 \\
\hline 22 & 312 & Phytanoyl-CoA dioxygenase & [Penicillium griseofulvum] (38/59) & KXG48658.1 \\
\hline 23 & 620 & oxidoreductase & [Malbranchea aurantiaca] $(49 / 65) ;$ malF & AGA37266.1 \\
\hline 24 & 293 & NmrA-like transcriptional regulator & [Penicillium roqueforti FM164] (71/82) & CDM28291.1 \\
\hline 25 & 246 & short-chain dehydrogenase & [Penicillium occitanis] $(60 / 68)$ & PCG98875.1 \\
\hline 26 & 73 & hypothetical protein CFD26_02699 & [Aspergillus turcosus] $(100 / 100)$ & OXN18438.1 \\
\hline 27 & 247 & NUDIX family hydrolase, putative & [Aspergillus fischeri NRRL 181] (80/89) & XP_001261565.1 \\
\hline 28 & 208 & endoglucanase-1 & [Aspergillus lentulus] $(83 / 89)$ & GAQ05884.1 \\
\hline
\end{tabular}




\begin{tabular}{|c|c|c|c|c|}
\hline 29 & 852 & glycosyl hydrolase, putative & [Aspergillus fischeri NRRL 181] (91/94) & XP_001261562.1 \\
\hline 30 & 406 & $\begin{array}{l}\text { ankyrin repeat domain-containing } \\
\text { protein } 50\end{array}$ & [Aspergillus udagawae] $(67 / 82)$ & GAO86765.1 \\
\hline \multicolumn{5}{|c|}{ Penicillium griseofulvum (GenBank accession number LHQR01000065) } \\
\hline ORF & $\begin{array}{l}\text { Size } \\
\text { (aa) }\end{array}$ & Putative Function & Relative identity/similarity (\%) & Accession No. \\
\hline 1 & 711 & $\begin{array}{c}\text { glycogen/starch/alpha-glucan } \\
\text { phosphorylase }\end{array}$ & [Penicillium griseofulvum] (96/96) & KXG49065.1 \\
\hline 2 & 356 & fungal G-protein, alpha subunit & [Penicillium griseofulvum] & KXG49066.1 \\
\hline 3 & 368 & MAP kinase SakA & [Penicillium digitatum PHI26] (98/99) & EKV06178.1 \\
\hline 4 & 809 & late secretory pathway protein AVL9 & [Penicillium griseofulvum] $(100 / 100)$ & KXG49068.1 \\
\hline 5 & 785 & Cullin homology & [Penicillium griseofulvum] $(100 / 100)$ & KXG49069.1 \\
\hline 6 & 2422 & NRPS & [Penicillium fellutanum] (37/54); phqB & AGA37269.1 \\
\hline 7 & 302 & NmrA-like family protein & [Aspergillus niger] & GAQ40480.1 \\
\hline 8 & 381 & O-methyltransferase & [Coccidioides posadasii str. Silveira] (33/47) & EFW19547.1 \\
\hline 9 & 452 & cytochrome P450 & [Penicillium griseofulvum] (96/96) & KXG49073.1 \\
\hline 10 & 470 & monooxygenase, FAD-binding & [Penicillium griseofulvum] $(100 / 100)$ & $\begin{array}{l}\text { KXG49074.1 } \\
\text { AOC } 84388.1 \\
\text { AGC83573.1 }\end{array}$ \\
\hline 11 & 394 & P450 monooxygenase & [Penicillium fellutanum] $(44 / 63) ; p h q M$ & $\begin{array}{l}\text { AGA37280.1 } \\
\text { KXG49075.1 }\end{array}$ \\
\hline 12 & 452 & P450 monooxygenase & [Penicillium fellutanum $](38 / 55) ; p h q L$ & $\begin{array}{l}\text { AGA37279.1 } \\
\text { KXG49076.1 }\end{array}$ \\
\hline 13 & 387 & cytochrome P450 & [Penicillium griseofulvum] $(100 / 100)$ & KXG49078.1 \\
\hline 14 & 336 & cytochrome P450 & [Penicillium griseofulvum] $(100 / 100)$ & KXG49078.1 \\
\hline 15 & 618 & oxidoreductase & [Penicillium fellutanum] (68/78); phqH & AGA37275.1 \\
\hline 16 & 383 & prenyltransferase & [Penicillium fellutanum] (44/62) & $\begin{array}{l}\text { AGA37277.1 } \\
\text { KXG49080.1 } \\
\end{array}$ \\
\hline $17-1$ & 383 & short-chain dehydrogenase & {$[$ Malbranchea aurantiaca $](51 / 73) ;$ malC } & AGA37263.1 \\
\hline $17-2$ & 462 & prenyltransferase & [Penicillium fellutanum] (82/87); phqI & AGA37276.1 \\
\hline 18 & 178 & $\mathrm{Hp}$ & [Penicillium griseofulvum] $(100 / 100)$ & KXG49083.1 \\
\hline 19 & 369 & $\mathrm{Hp}$ & [Penicillium griseofulvum] $(100 / 100)$ & KXG49084.1 \\
\hline 20 & 274 & $\mathrm{Hp}$ & [Penicillium griseofulvum] (94/93) & KXG49085.1 \\
\hline 21 & 404 & Calcium-binding EF-hand & [Penicillium griseofulvum] $(100 / 100)$ & KXG49086.1 \\
\hline 22 & 214 & pectate lyase, catalytic & [Penicillium griseofulvum] $(89 / 89)$ & KXG49087.1 \\
\hline 23 & 884 & SNF2-related protein & [Penicillium griseofulvum] (98/97) & KXG49088.1 \\
\hline
\end{tabular}

